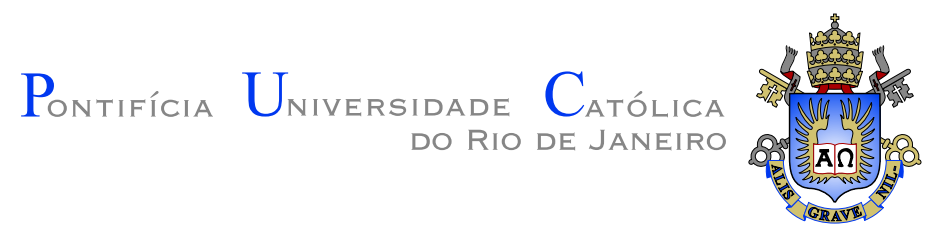

Flávio Henrique Marchesini de Oliveira

Rheology of Waxy Crude Oils

Tese de Doutorado

Thesis presented to the Programa de Pós-graduação em Engenharia Mecânica of the Departamento de Engenharia Mecânica, PUC-Rio, as partial fulfillment of the requirements for the degree of Doutor em Engenharia Mecânica

Advisor: Prof. Paulo Roberto de Souza Mendes 


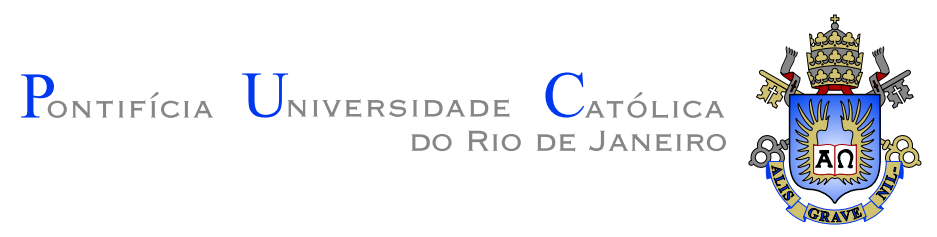

Flávio Henrique Marchesini de Oliveira

\section{Rheology of Waxy Crude Oils}

Thesis presented to the Programa de Pós-graduação em Engenharia Mecânica of the Departamento de Engenharia Mecânica do Centro Técnico Científico da PUC-Rio, as partial fulfillment of the requirements for the degree of Doutor em Engenharia Mecânica. Approved by the following commission:

Prof. Paulo Roberto de Souza Mendes

Advisor

Departamento de Engenharia Mecânica - PUC-Rio

Prof. Luís Fernando Alzuguir Azevedo Departamento de Engenharia Mecânica - PUC-Rio

Prof. Roney Leon Thompson Departamento de Engenharia Mecânica — UFF

Prof. Márcio Nele de Souza PEQ/COPPE - UFRJ

Dr. Márcia Cristina Khalil de Oliveira CENPES - PETROBRAS

Prof. José Eugênio Leal

Coordinator of the Centro Técnico Científico da PUC-Rio 
All rights reserved.

\section{Flávio Henrique Marchesini de Oliveira}

Flávio H. Marchesini graduated in Chemical Engineering from Federal University of Rio de Janeiro (UFRJ) in 2004. Then, he started to work with Rheology and non-Newtonian Continuum Mechanics in the Rheology Group (GReo) at PUC-Rio, where he obtained his Masters degree in Mechanical Engineering in 2008. He is currently at Halliburton as a Senior Engineer.

Bibliographic data

Marchesini, Flávio Henrique

Rheology of Waxy Crude Oils / Flávio Henrique Marchesini de Oliveira; advisor: Paulo Roberto de Souza Mendes 2012.

94 f.: il. (color); $29,7 \mathrm{~cm}$

Tese (doutorado) - Pontifícia Universidade Católica do Rio de Janeiro, Departamento de Engenharia Mecânica, 2012.

Inclui Bibliografia.

1. Engenharia Mecânica - Teses. 2. Reologia. 3. Óleos parafínicos crus. 4. Reometria. 5. Tensão limite de escoamento. 6. Garantia de escoamento. 7. Início de escoamento. I. de Souza Mendes, Paulo Roberto. II. Pontifícia Universidade Católica do Rio de Janeiro. Departamento de Engenharia Mecânica. III. Título. 


\section{Acknowledgments}

This thesis has been produced by research efforts expended since the third quarter of 2008. During this period I received support to complete this work, learned and worked with different persons to whom I would like to express here my sincere gratitude.

First, I would like to thank my family, in particular, my mother and all of my friends, specially Rodrigo Junqueira, Raphael Chagas, Paula Marchesini, Luísa Marchesini, Paula Mey and Apoena Frota for all incentive, support, and apprenticeship during this period. All the good moments with them, for sure, were fundamental to keep the motivation to complete this work. I would like also to thank my colleagues and students from Rheology Group at PUC-Rio, in particular Bruna Leopércio for performing some experiments presented in this thesis and my friend Bruno Fonseca for his partnership.

I am very grateful to Cláudio Ziglio from PETROBRAS, not only for the financial support, but also for bringing us this interesting problem and for all collaboration and partnership during this research. I am indebted to $\mathrm{CNPq}$ for also providing financial support to this research. In addition, I would like to thank Jacob Thomas, Ashok Santra, Maria Paiva, and Kris Ravi for their incentive, support, and comprehension since I started to work at Halliburton during the end of this journey.

I acknowledge with deep appreciation one of my best friends and partner at GReo, Priscilla Varges, for her friendship, technical discussions, and all help with the bureaucracy and work at PUC-Rio. Without her help it would have been very difficult to expend the required time to complete this work.

I am particularly indebted to my advisor Paulo de Souza Mendes to whom I owe so much! He was fundamental to my education and my life, providing me exposure to high level international research and all the support, guidance, and incentive necessary to complete this work. It is a great honor to be his former student. 
Finally, but foremost, I would like to express my sincere gratitude to my girlfriend Alexandra Alicke, who I love so much! I met her four years ago when I was trying to find a smart undergraduate student to help me with this research. At that time, one day Priscilla Varges told me: "I found the perfect person to help you!" When she started to work with me, she have never heard about rheology. I started to teach her at that time and without her professional dedication, passion, support, suggestions, and motivation it would be impossible to perform the more than 1000 rheometric experiments summarized in this thesis and to achieve the same level of understanding of the subject. I am very proud of her! Nowadays, she can deeply discuss the rheology of waxy oils with anybody and, more interesting, she sometimes disagrees with me in certain aspects. We have learned a lot together during this period, have had unforgettable moments and this thesis is dedicated to her! 


\section{Resumo}

Marchesini, Flávio Henrique; de Souza Mendes, Paulo Roberto. Reologia de Óleos Parafínicos Crus. Rio de Janeiro, 2012. 94p. Tese de Doutorado - Departamento de Engenharia Mecânica, Pontifícia Universidade Católica do Rio de Janeiro.

Esta tese é sobre a reologia de óleos parafínicos crus. Um protocolo baseado na literatura relevante é desenvolvido para garantir que históricos térmico e de cisalhamento bem definidos sejam impostos em amostras que serão submetidas à medições reológicas. Com este protocolo, uma caracterização reológica quase completa de um óleo parafínico cru brasileiro é executada . Quatro experimentos reométricos são realizados, a saber, escoamento em estado estacionário, taxa de cisalhamento constante, tensão constante e rampa oscilatória de amplitude de tensão. Propriedades reológicas importantes, tais como, viscosidade, tensão limite de escoamento e módulos elástico e viscoso são medidos com o auxílio de técnicas diferentes em uma ampla faixa de condições. Além disso, uma comparação entre essas técnicas de medição é realizada. O processo de quebra da microestrutura do óleo parafínico brasileiro, assim como os efeitos na reologia do histórico do material e do tempo de repouso isotérmico são estudados. Fenômenos interessantes são observados, incluindo bifurcação da taxa de cisalhamento, dependência da tensão limite com os históricos térmico e de cisalhamento e independência dos resultados de regime permanente com relação à condição inicial após o resfriamento. Por fim, oportunidades para futuros desenvolvimentos e investigações são enfatizadas.

\section{Palavras-chave}

Reologia; Óleos parafínicos crus; Reometria; Tensão limite de escoamento; Garantia de escoamento; Início de escoamento. 


\section{Abstract}

Marchesini, Flávio Henrique; de Souza Mendes, Paulo Roberto (Advisor). Rheology of Waxy Crude Oils. Rio de Janeiro, 2012. 94p. D.Sc. Thesis — Departamento de Engenharia Mecânica, Pontifícia Universidade Católica do Rio de Janeiro.

This thesis deals with the rheology of waxy crude oils. A protocol based on the relevant literature is developed to guarantee well defined shear and thermal histories to waxy crude oil samples to be submitted to rheological measurements. With this protocol, a fair complete rheological characterization of a Brazilian waxy crude oil is performed. Four rheometric experiments, namely steady-state flow, constant shear rate, constant shear stress, and dynamic-stress-amplitude sweep are carried out. Important rheological properties, such as, viscosity, yield stress, and storage and loss moduli are measured with different techniques in a wide range of conditions and a comparison between the techniques is presented. The yielding as well as the history effects and the effects of isothermal holding time on the microstructure and rheology of a Brazilian waxy crude oil are investigated. Interesting phenomena are observed, including bifurcation of shear rate, dependence of the yield stress with thermal and shear histories, and independence of the steady-state results with the post-cooling initial condition of the experiment. At last, opportunities for further developments are highlighted.

\section{Keywords}

Rheology; Waxy crude oils; Rheometry; Yield stress; Flow assurance; Start-up flows. 


\section{Contents}

1 Introduction $\quad 13$

$\begin{array}{lll}1.1 & \text { Motivation } & 13\end{array}$

$\begin{array}{lll}1.2 & \text { Research objectives } & 14\end{array}$

$\begin{array}{lll}1.3 & \text { Thesis overview } & 15\end{array}$

$\begin{array}{lll}1.4 & \text { Published work } & 16\end{array}$

2 Sample Preparation $\quad 18$

2.1 Introduction 18

2.2 Materials and methods 21

2.3 Sample preparation itself 22

Pretreatment $\quad 22$

Isothermal holding time $\quad 23$

Cooling process $\quad 23$

2.4 Determining the test conditions: the thermal cycle test 24

Description of the thermal cycle test 24

$\begin{array}{ll}\text { Characteristic temperatures } & 24\end{array}$

$\begin{array}{ll}\text { Choice of the geometry } & 27\end{array}$

Influence of the gap $\quad 30$

Choice of the starting temperature $T_{i}$

$\begin{array}{lll}2.5 & \text { Reliability and sources of error } & 37\end{array}$

Repeatability $\quad 37$

Systematic errors 38

Chemical stability $\quad 39$

Apparent wall slip and adhesive failure $\quad 40$

Other sources of error $\quad 44$

2.6 Final remarks $\quad 45$

$3 \quad$ Post-cooling transient and steady-state measurements $\quad 47$

$\begin{array}{lll}3.1 & \text { Introduction } & 47\end{array}$

3.2 Yielding and yield-stress measurements: an historical survey 48

3.3 Materials and methods $\quad 53$

3.4 Results and discussion 53

Constant shear stress rheometric experiments $\quad 54$

Statically cooled samples $\quad 54$

Samples cooled under shear $\quad 56$

Constant shear rate rheometric experiments $\quad 60$

Steady-state flow rheometric experiments 62

$\begin{array}{lll}3.5 & \text { Final remarks } & 63\end{array}$

4 Post-cooling oscillatory measurements $\quad 65$

$\begin{array}{lll}4.1 & \text { Introduction } & 65\end{array}$ 
4.2 Materials and methods $\quad 66$

4.3 Results and discussion 66

Description of the post-cooling stress amplitude sweep test and its main parameters 66

Effects of shear and thermal histories on structure and rheology 68

Effects of holding time on structure and rheology $\quad 72$

Holding time before yielding $\quad 72$

Holding time after yielding 74

Overview $\quad 76$

Effects of frequency on structure and rheology $\quad 76$

$\begin{array}{lll}4.4 & \text { Final remarks } & 79\end{array}$

5 Concluding Remarks $\quad 81$

5.1 Suggestions for future research works 83

$\begin{array}{lr}\text { Bibliography } & 85\end{array}$ 


\section{List of Figures}

2.1 A thermal cycle test viscosity plot, with the definitions of $T_{c}$, $T_{d}, T_{i}, T_{0}, \eta_{h 0}$, and $\xi$ : (a) normal plot, and (b) inverse absolute temperature scale plot.

2.2 Thermal cycle test results for different geometries. 28

2.3 Thermal cycle test results for three different small-gap geometries. 29

2.4 Effect of the gap on the crystallization process and rheology: (a) $\dot{\gamma}_{h}=2 s^{-1}$; (b) $\dot{\gamma}_{h}=20 s^{-1}$; (c) $\dot{\gamma}_{h}=200 s^{-1}$.

2.5 Maximum viscosity $\eta_{h 0}$ and crystallization temperature $T_{c}$ as a function of the gap.

2.6 Effect of $T_{i}$ on the crystallization process and rheology. 35

$\begin{array}{lll}2.7 & \text { Temperature cycle - repeatability and reliability. } & 37\end{array}$

2.8 Comparison between data obtained with two different rheometers. 38

2.9 Results of the triple thermal cycle test. 39

2.10 Comparison between results obtained with smooth and crosshatched plates.

3.1 Post-cooling constant shear stress tests performed on statically cooled samples, $\dot{\gamma}_{h}=0 \mathrm{~s}^{-1}$ and $\dot{T}_{h}=1{ }^{\circ} \mathrm{C} / \mathrm{min}$ : (a) strain-versustime plot and (b) shear-rate-versus-time plot.

3.2 Post-cooling constant shear stress tests performed on samples cooled under shear, $\dot{\gamma}_{h}=20 \mathrm{~s}^{-1}$ and $\dot{T}_{h}=1{ }^{\circ} \mathrm{C} / \mathrm{min}$ : investigation of the effects of changing the initial condition on the results, (a) strain-versus-time plot and (b) shear-rate-versus-time plot.

3.3 Post-cooling constant shear stress tests performed on samples cooled under shear, $\dot{\gamma}_{h}=20 \mathrm{~s}^{-1}$ and $\dot{T}_{h}=1{ }^{\circ} \mathrm{C} / \mathrm{min}$ : (a) strainversus-time plot and (b) shear-rate-versus-time plot.

3.4 Post-cooling constant shear stress at $0.9 \mathrm{~Pa}$ applied on samples cooled under shear, $\dot{\gamma}_{h}=20 \mathrm{~s}^{-1}$ and $\dot{T}_{h}=1{ }^{\circ} \mathrm{C} / \mathrm{min}$ : (a) strainversus-time plot and (b) shear-rate-versus-time plot.

3.5 Post-cooling constant shear rate tests performed on samples cooled at $\dot{\gamma}_{h}=20 \mathrm{~s}^{-1}$ and $\dot{T}_{h}=1{ }^{\circ} \mathrm{C} / \mathrm{min}$.

3.6 Post-cooling flow curve of samples cooled at $\dot{\gamma}_{h}=20 \mathrm{~s}^{-1}$ and $\dot{T}_{h}$ $=1^{\circ} \mathrm{C} / \mathrm{min}$.

4.1 Stress-amplitude sweep test: (a) $G^{\prime}$ and $G^{\prime \prime}$ as a function of stress amplitude and (b) stress amplitude as a function of strain - main parameters.

4.2 Postcooling stress-amplitude-sweep test started immediately after reaching $T_{0}$ : effects of $\dot{\gamma}_{h} ; \dot{T}_{h}=0.1{ }^{\circ} \mathrm{C} / \mathrm{min}$.

4.3 Postcooling stress-amplitude-sweep test started immediately after reaching $T_{0}$ : effects of $\dot{\gamma}_{h} ; \dot{T}_{h}=1{ }^{\circ} \mathrm{C} / \mathrm{min}$.

4.4 Postcooling stress-amplitude-sweep test started immediately after reaching $T_{0}$ : effects of $\dot{T}_{h} ; \dot{\gamma}_{h}=0 \mathrm{~s}^{-1}$.

4.5 Postcooling stress-amplitude-sweep test started immediately after reaching $T_{0}$ : effects of $\dot{T}_{h} ; \dot{\gamma}_{h}=20 \mathrm{~s}^{-1}$. 
4.6 Stress-amplitude-sweep test started after reaching $T_{0}$ without post-cooling shear: effects of holding time before yielding; $\dot{\gamma}_{h}=$ $0 \mathrm{~s}^{-1}$ and $\dot{T}_{h}=1{ }^{\circ} \mathrm{C} / \mathrm{min}$.

4.7 Stress-amplitude-sweep test started after reaching $T_{0}$ without post-cooling shear: effects of holding time before yielding; $\dot{\gamma}_{h}=$ $20 \mathrm{~s}^{-1}$ and $\dot{T}_{h}=1{ }^{\circ} \mathrm{C} / \mathrm{min}$.

4.8 Stress-amplitude-sweep test started after $30 \mathrm{~min}$ of postcooling shear at $20 \mathrm{~s}^{-1}$ : effects of holding time; $\dot{\gamma}_{h}=0 \mathrm{~s}^{-1}$ and $\dot{T}_{h}=$ $1{ }^{\circ} \mathrm{C} / \mathrm{min}$.

4.9 Stress-amplitude-sweep test started after $30 \mathrm{~min}$ of postcooling shear at $20 \mathrm{~s}^{-1}$ : effects of holding time; $\dot{\gamma}_{h}=20 \mathrm{~s}^{-1}$ and $\dot{T}_{h}=$ $1{ }^{\circ} \mathrm{C} / \mathrm{min}$.

4.10 Postcooling stress-amplitude-sweep test started immediately after reaching $T_{0}$ : effects of frequency; $\dot{\gamma}_{h}=0 \mathrm{~s}^{-1}$ and $\dot{T}_{h}=1{ }^{\circ} \mathrm{C} / \mathrm{min}$.

4.11 Postcooling stress-amplitude-sweep test started immediately after 77 reaching $T_{0}$ : effects of frequency; $\dot{\gamma}_{h}=20 \mathrm{~s}^{-1}$ and $\dot{T}_{h}=1{ }^{\circ} \mathrm{C} / \mathrm{min} . \quad 78$ 
"There is a great desire on me on improving, getting better. That makes me happy. And every time that I feel that I am slowing down my learning process, my learning curve is getting flat or whatever, than, it doesn't make me very happy. And that applies not only as a professional, as a racing driver, but also as a man. Of course, I shall have a lot more to learn as a man than a racing driver because my career could not lasts many years. My life, hopefully, will go still for a long time. Maybe I'm only on the half of my life right now. So, that is a lot to go, a lot to learn, a lot to do still in life and happiness will come when I feel complete as a whole, which definitely I do not feel today, but I have plenty of time to fulfill that too."

Ayrton Senna da Silva 


\section{Introduction}

This is the introductory chapter of the thesis entitled "Rheology of Waxy Crude Oils" produced at Pontifícia Universidade Católica do Rio de Janeiro, PUC-Rio. In this chapter, we describe the motivation and research objectives (Secs. 1.1 and 1.2) as well as present an overview of the thesis (Sec. 1.3). In addition, we list the work presented in different conferences, the work to be published, and the published work related to this thesis (Sec. 1.4).

\section{1}

\section{Motivation}

Wax crystallization is widely related as one of the most important problems inherent to the production, storage and processing of waxy oils to both lubricant and petroleum industries. In particular, there are two main problems that arise from handling waxy oils in pipelines or in different equipments: deposition of wax crystals and gelation [1,2].

Deposition occurs during the flow of these materials, when wax crystals can adhere to cold surfaces and form a deposit layer that is frequently hard to be removed $[3,4,5,6]$. This phenomenon is a serious issue that can lead to pipeline blockages and has been discussed and modeled in a number of papers in a research effort to understand the mechanisms responsible for wax deposition $[7,8,9,10,11,12,13,14,15,16,17,18,19,20,21,22,23,24,25,26,27,28,29]$.

Gelation of waxy oils is a major problem regarding the flow assurance in oil production and transportation, because this phenomenon is associated to important changes in rheology. Therefore, the start-up (or restart) and lineclearing of gelled oil pipelines require the accurate knowledge of the rheological properties of the gelled crude oil to be transported, especially the yield stress. This is particularly true in a deepwater scenario and has becoming more and more important as oil exploration advance to higher depths and arctic regions [30].

Gelation is also a problem to lubricant industry with regard to machinery lubrication maintenance and the necessity of formulate lubricants with acceptable low temperature performance. [31] 
The gelation process occurs because of crystallization of wax. The solubility of wax compounds decreases as the temperature is lowered, leading to precipitation of wax crystals $[6,32]$. During this cooling process, when a certain amount of wax crystals appears in a way that the interaction between them becomes important, an interlocking network of wax crystals start to develop, and consequently, remarkable effects on the rheological properties of these oils can be observed. At this point, it is possible to define a crystallization temperature $\left(T_{c}\right)$, as the temperature below which a sharp increase in viscosity is observed [31, 32, 33, 34].

From the rheological viewpoint, above this temperature $T_{c}$, waxy oils are simple Newtonian liquids, presenting the typical Arrhenius viscositytemperature dependence. However, below $T_{c}$, the interlocking network of wax crystals gives rise to an extremely complex non-Newtonian behavior, that includes dependence of the rheological properties not only upon time, temperature, and shear rate as usually observed in complex fluids, but also upon the temperature and shear histories experienced by the oil sample $[1,33,35]$.

Because of the importance of determining the rheological properties of these materials under different circumstances, many researchers worked over the last decades to understand the microstructure and rheology of waxy oils. Considerable progress has been made in this subject, although some lack of understanding still exists with regard to (i) the measurement procedures employed in rheological tests, (ii) the yielding of waxy crude gels, (iii) the history effects on the rheological properties of crude oil gels, and (iv) the effects of isothermal holding time on the microstructure and rheology of crude oil gels. The extremely complex rheological behavior of gelled waxy oils constitutes an interesting scientific challenge, so that more investigation is required.

\section{2}

\section{Research objectives}

This thesis is concerned with the rheology of waxy crude oils, specially at low enough temperatures, condition, in which, the gelation phenomenon is observed. The main objectives of this research are:

- to establish appropriate protocols for preparing samples of waxy crude oils for rheological characterization;

- to perform accurate measurements of the yield stress(es) of a Brazilian waxy crude oil with different techniques in a wide range of conditions, and evaluate which technique is more practical and reliable; 
- to measure other relevant rheological properties of the waxy crude oil investigated, such as viscosity, storage modulus $\left(G^{\prime}\right)$, and loss modulus $\left(G^{\prime \prime}\right)$ in a wide range of conditions;

- to investigate the yielding of a Brazilian waxy crude oil;

- to investigate the effects of the isothermal holding time and shear and thermal histories on the microstructure and rheology of the Brazilian waxy crude oil.

\section{3}

\section{Thesis overview}

This thesis is divided in five chapters. As previously stated, the motivation and objectives of this research, as well as, an overview of the thesis is given in Chapter 1. In addition, a list of the published work related to this thesis is also found in Chapter 1. The following three chapters are written in such way to enable the reader to find all the required information for understanding the discussed subject enclosed in each chapter. Therefore, each one of these three chapters can be read independently, depending upon the background of the reader. Besides that, these chapters are organized to cover a multitude of phenomena related to the rheology of waxy oils, from the more fundamental to the more complex phenomena.

In Chapter 2, the protocols developed for preparing samples of waxy crude oils to be submitted to rheological characterization are discussed. In addition, this chapter also gives an idea of the complexity of the rheology of waxy oils as well as discuss the importance of the cooling process to the post-cooling microstructure and rheology of these materials.

The results of post-cooling transient and steady-state rheometric measurements are shown in Chapter 3. Two different ways of measuring the yield stress(es) of these materials are compared, namely, bifurcation and extrapolation from steady-state data. In addition, two flow curves of the Brazilian waxy crude oil are presented for both samples cooled statically or under the application of a shear rate.

The results of post-cooling oscillatory rheometric measurements are shown in Chapter 4. Another way of measuring the yield stress(es) of gelled waxy oils is evaluated. Moreover, the yielding and the effects of isothermal holding time as well as the effects of thermal and shear histories on the oscillatory rheological properties are investigated.

The main conclusions of the present research are summarized in Chapter 5. The precautions required during the preparation of the sample of a waxy 
crude oil to obtain meaningful rheological data are also emphasized. In addition, the different rheometric techniques for measuring the yield stress(es) of these materials are compared. At last, opportunities for further developments in this field are highlighted in this chapter.

\section{4}

\section{Published work}

In this section, we itemize the work presented in different conferences, the work to be published, and the published work related to this thesis.

- Published work in peer-review journals and work to be published:

- Marchesini, F.H., Alicke, A.A., de Souza Mendes, P.R., Ziglio, C.M., "Rheological Characterization of Waxy Crude Oils: Sample Preparation", Energy \&6 Fuels, 26(5), 2566-2577, 2012.

- Marchesini, F.H., Alicke, A.A., de Souza Mendes, P.R., Leopércio, B.C., "Rheological Characterization of Waxy Crude Oils: (1) Transient and Steady-State Measurements", Journal of Rheology, in preparation.

- Marchesini, F.H., Alicke, A.A., de Souza Mendes, P.R., "Rheological Characterization of Waxy Crude Oils: (2) Oscillatory Measurements", Journal of Rheology, in preparation.

- Work presented in conferences:

- Marchesini, F.H., Alicke, A.A., de Souza Mendes, P.R., Leopércio, B.C., "Yielding of waxy crude oils revisited", XVI International Congress on Rheology, 2012.

- Marchesini, F.H., Alicke, A.A., de Souza Mendes, P.R., "Rheology of waxy crude oils", 4th Workshop on Viscoplastic Fluids: from Theory to Application, 2011.

- Marchesini, F.H., Alicke, A.A., de Souza Mendes, P.R., Ziglio, C.M., "Rheological characterization of waxy crude oils", 83rd Annual Meeting of the Society of Rheology, 2011.

- Marchesini, F.H., Alicke, A.A., de Souza Mendes, P.R., Ziglio, C.M., "Rheology of waxy oils: cooling process, yielding, history effects and post-cooling flow properties", 12th PETROPHASE, 2011.

- Marchesini, F.H., de Souza Mendes, P.R., Nieckele, A.O., Azevedo, L.F.A., Trampus, B.C., Minchola, L.R. "Post-cooling flow properties of a model waxy crude oil", rth Annual European Rheology Conference, 2011. 
- Marchesini, F.H., Alicke, A.A., de Souza Mendes, P.R., Ziglio, C.M., "History effects and post-cooling flow properties of waxy oils", 82nd Annual Meeting of the Society of Rheology, 2010.

- Marchesini, F.H., Alicke, A.A., de Souza Mendes, P.R., Ziglio, C.M., "Rheology of Waxy Oils", V Brazilian Conference on Rheology, 2010.

- Marchesini, F.H., Alicke, A.A., de Souza Mendes, P.R., Ziglio, C.M., "Waxy Crude Oils: rheological characterization, apparent wall slip, and history effects", 6th Annual European Rheology Conference, 2010.

- Marchesini, F.H., Alicke, A.A., de Souza Mendes, P.R., Ziglio, C.M., "Transient rheology of paraffinic oils at low temperature", 10th PETROPHASE, 2009. 


\section{2}

\section{Sample Preparation}

In this chapter we describe an experimental protocol designed to ensure well defined thermal and shear histories of a waxy crude oil sample to be submitted to rheological measurements. We discuss the criteria for choosing an appropriate pretreatment, the geometry, and the temperature range, among other parameters. This protocol includes the so-called thermal cycle test, which is shown to play a key role in determining the appropriate test conditions. Possible sources of measurement error are discussed in detail. The contents of this chapter are published on Energy \& Fuels 2012 [36].

\section{1}

\section{Introduction}

Deepwater and ultra-deepwater oil production have grown fast over the last few decades. In this activity, the extracted oil is exposed to low temperatures, because the temperature of sea floor is around $4{ }^{\circ} \mathrm{C}$ in the presently explored depths. In the event of a long production shutdown, the oil temperature may drop severely, leading to wax crystallization, followed by gelation and, hence, a radical change in the rheological behavior.

While waxy oils are simple Newtonian liquids at higher temperatures, they become highly complex non-Newtonian materials below the crystallization temperature $[33,34]$. This phenomenon complicates the operation and design of a pipeline system [34, 37], because of the necessity of determining the rheological properties of waxy crude oils at low temperatures. Thus, the measurement of reliable rheological properties of these oils is a fundamental issue in the petroleum industry, especially regarding flow assurance.

During the 1970s, a significant research effort has been devoted to improve the techniques for measuring the rheological properties of gelled waxy oils [35, 38, 39, 40, 41, 42, 43, 44, 1, 45]. Davenport and Somper [35] performed an extensive investigation, including experiments in full-scale, pilot-scale, and laboratory-model pipelines, as well as in a rotational viscometer with the Couette geometry. As possible sources of error in rheological measurements, they mentioned (i) the loss of light ends during the course of experiments, 
(ii) small changes in thermal and shear histories from sample to sample, and (iii) gap dependency in transient measurements. Perkins and Turner [38] also used the Couette viscometry to study the rheology of the Prudhoe Bay oil. They listed as factors that significantly affect the yield stress of a gelled waxy oil (i) the thermal and shear history, (ii) aging, and (iii) composition. The dependence upon such a large number of parameters renders the rheological measurements rather difficult, and consequently, comparisons between data obtained with different techniques and instruments were typically poor.

Petrellis and Flumerfelt [44] used a Rheogoniometer with cone and plate, and Couette geometries to investigate the equilibrium and time-dependent rheology of different crude oils under controlled shear and thermal conditions. They reported that below the pour point waxy oils are irreversible shear debradable materials with slight recovery after shear and a complete recovery after reheating and re-cooling.

Smith and Ramsden [1] also carried out experiments in a rotational viscometer and full-scale, pilot-scale, and laboratory-model pipelines. In addition, they performed pour point tests and emphasized the importance of applying a pretreatment to the samples in yield stress measurements at low temperatures. They pointed out that only by reproducing both thermal and mechanical conditions experienced in production is it possible to correctly simulate a full scale line. Moreover, they highlighted that, in temperatures at which non-Newtonian behavior is observed, the rheological properties of waxy crude oils are determined by the amount, size, and shape of wax crystals, which, in turn, are dependent upon (i) the solvent power of the liquid phase, (ii) the temperature, (iii) the thermal and shear histories, and (iv) the presence of crystal form modifiers, including natural asphaltenes, resins, and artificial flow improver additives.

Besides the previously mentioned techniques, capillary viscometry was also used in the past as an attempt to measure the rheological properties of gelled waxy oils [33, 46, 47, 48, 49, 50]. However, it was only in the late 1980s/early 1990s that a better control of both shear and thermal histories was introduced in the experiments, so that repeatable and more reliable results could be obtained.

In seminal papers, Wardhaugh and Boger [33, 34] described a systematic methodology to obtain repeatable results from a rotational rheometer technique. They [46] showed that rotational rheometry is more suitable and accurate for the rheological characterization of gelled waxy oils and obtained good agreement between steady-state data for a Jackson-Hutton crude using both Couette and cone-and-plate geometries, each with a different rheometer 
$[33,34]$. Moreover, they discussed in detail the possible sources of error in rheological measurements involving waxy oils.

Essentially, the method described by Wardhaugh and Boger [33, 34] consists of heating the oil sample above the wax appearance temperature (WAT) for a sufficient amount of time to erase the thermal memory of the oil by redissolving wax and asphaltenes and then cooling the sample to the measurement temperature $T_{0}$ under strict control of shear and cooling rates, as already recommended by Petrellis and Flumerfelt [44], and Smith and Ramsden [1]. If this procedure is followed, then a reliable flow curve can be obtained and "realistic comparisons made between different instruments" [33, 34].

Rønningsen [3] also used the cone-and-plate rheometry to study the rheology of North Sea crude oils. He compared the results to model pipeline experiments and came up with the conclusion that rotational rheometry is a "useful supplement to model pipeline tests, at least for comparative purposes." After the papers by Wardhaugh and Boger [33, 34] and Rønningsen [3], the use of cone-and-plate rheometry became frequent $[51,52,53,12,54,6,55]$, based on the fact that it is the only geometry capable of keeping the same shear history throughout the sample, because the flow is homogeneous within this geometry. In addition, because of the small sample required, the thermal history is easily controllable [52].

It is important noting that different authors [33, 35, 44, 3, 31] observed more repeatability in steady-state data. That is, it is observed that equilibrium flow properties of gelled waxy oils are less influenced by experimental conditions and history effects than the transient properties. Hence, the agreement between steady-state data for a single crude oil obtained from two different viscometric flows and rheometers is not sufficient evidence to elect the cone and plate as the standard geometry for rheological characterization of all gelled waxy oils. In fact, at low temperatures, it is usually reported in the literature that high values of yield stress and viscosity are obtained with small-gap geometries [33, 35, 1, 46, 3]. Wardhaugh and Boger [33] themselves obtained a higher viscosity level in cooling ramps performed with a cone-and-plate geometry when compared to Couette results. They commented that "techniques for measurement of the equilibrium flow properties have now been established, but the same is not true of yield stress and the subsequent time-dependent properties." As far as the present authors know, a systematic study about the measurement procedures employed for defining an appropriate shear and thermal histories in a waxy oil sample to be submitted to rheological characterization is not available in the literature. Therefore, more investigation is required. 
In this chapter, we present in detail a procedure for preparing waxy crude oil samples for rheological measurements. This procedure proved successful in granting an excellent level of repeatability of rheological data, even in transient rheological tests.

The procedure may be divided in three main steps. The first step, usually denominated pretreatment, consists of applying a thermal treatment to the whole batch of oil, aiming at the achievement of a stable chemical composition. The second step is a isothermal holding time, performed to dissolve all wax crystals in the oil. The third step is the cooling process, to which each sample should be submitted immediately before the beginning of any rheological measurement to achieve the measurement temperature with fixed shear and thermal histories, as recommended in the literature [33, 34, 44, 1, 52, 53]. The three steps of the procedure are explained in detail later on in this text.

We then describe in detail how to use the so-called thermal cycle tests to determine the appropriate geometry, gap, and temperature range to be used in future rheological measurements. Finally, we discuss repeatability, reliability, and different sources of error.

\section{2}

\section{Materials and methods}

A Brazilian waxy crude oil provided by PETROBRAS was used in the research described in this thesis. All the results and conclusions presented in this chapter were obtained with the aid of two rotational rheometers.

One was an AR-G2 rheometer from TA instruments, with the following geometries:

1. cone and plate, $40 \mathrm{~mm}$ diameter, $2^{\circ}$ angle, and truncation of $59 \mu \mathrm{m}$;

2. cone and plate, $60 \mathrm{~mm}$ diameter, $1^{\circ}$ angle, and truncation of $24 \mu \mathrm{m}$;

3. Couette with recessed end, $30 \mathrm{~mm}$ cup diameter and $27.8 \mathrm{~mm}$ bob diameter;

4. smooth parallel plates, $60 \mathrm{~mm}$ diameter, with gaps ranging within 0.5 and $3 \mathrm{~mm}$;

5. cross hatched parallel plates, $60 \mathrm{~mm}$ diameter, with the same gap range.

The other rheometer used in the investigation was a MCR-501 from Anton Paar, with $50 \mathrm{~mm}$ diameter smooth parallel plates and the same gap range used in the AR-G2. The temperature control in both rheometers was through Peltier systems. 
During all the rheometric tests performed, a cover was used to minimize evaporation of light ends and. consequently, to reduce compositional changes.

To provide samples from the same batch throughout the whole experimental investigation - which spanned for several months - the batch of pretreated crude oil at $60^{\circ} \mathrm{C}$ was distributed in twelve closed bottles, with each one containing $450 \mathrm{~mL}$, and then stored at room temperature $\left(\approx 23^{\circ} \mathrm{C}\right)$.

Before each rheological test, one of the bottles containing the crude oil was homogenized, and using a syringe, a sample was then collected from this bottle and loaded into the rheometer whose temperature had been previously set to $25^{\circ} \mathrm{C}$.

\section{3}

\section{Sample preparation itself}

In this section we itemize the steps that we deem necessary in the preparation of a sample for a rheological measurement, presenting justifications and comparisons with the procedures recommended in the related literature.

\subsection{1}

\section{Pretreatment}

The first step of the sample preparation is to determine the appropriate pretreatment to be applied to the samples. Petrellis and Flumerfelt [44], and Wardhaugh and Boger $[33,34]$ pointed out that a stable chemical composition must be ensured before the rheological measurements. The method used by Wardhaugh and Boger [33, 34] to achieve a stable chemical composition was to impose heating to the crude in an open bottle, to evaporate light ends. As mentioned by these authors themselves, this method can cause non-negligible changes in composition.

Smith and Ramsden [1] emphasized the importance of applying a pretreatment within a temperature range representative of the process of interest. The pretreatment should not involve heating at temperatures higher than the ones occurring during the industrial process, to prevent significant effects, which would not be present in the industrial process, of resins and asphaltenes on wax crystallization while cooling the crude again. Asphaltenes and resins are the most polar fractions of crude oils and interact with wax forming crystals, affecting wax crystallization [1]. Venkatesan et al. [56] pointed out that asphaltenes act on the wax crystal network, making it less entangled, thereby decreasing the yield stress.

The pretreatment applied to our crude oil was devised to follow as closely as possible these guidelines. It consists of heating the crude at $50^{\circ} \mathrm{C}$ for $3 \mathrm{~h}$ in 
an open bottle, then raising the temperature to $60^{\circ} \mathrm{C}$, the maximum process temperature in our case, and continue heating for another $1 \mathrm{~h}$. This pretreatment promotes loss of the light ends, thus ensuring a stable composition during testing.

\subsection{2}

\section{Isothermal holding time}

The second step of sample preparation is the isothermal holding time. One of the bottles containing the crude oil is homogenized, and then, using a syringe, a sample is collected from this bottle and loaded into the rheometer, whose temperature was previously set to $25^{\circ} \mathrm{C}$. The rheometer temperature is then raised to an initial value called the starting temperature $T_{i}$, which is achieved in a few seconds with a Peltier system. The temperature is kept at $T_{i}$ for $30 \mathrm{~min}$ in order to redissolve all the wax crystals that may have precipitated at room temperature, thus erasing the thermal memory [33, 34].

This procedure differs from the procedure commonly adopted by the flow assurance community, namely, heating the whole bottle to above the WAT before collecting a sample to be loaded into the previously heated rheometer. Note that, after a large number of tests, reheating the whole bottle every time before collecting a sample can introduce significant irreversible changes in composition, because of thermal cycling effects. Thus, this procedure seems to be appropriate for a limited number of tests only or else if the pretreatment used is strong enough to guarantee a very stable chemical composition.

In our case, because the number of tests was very large, it was necessary to adopt the modified procedure described above. It is important noting that we always obtained essentially the same result from the first to the last sample taken from the same bottle, showing that the modified procedure provides samples that are representative of the bulk of the oil. Therefore, our experience acquired with over 1000 tests strongly suggests that, at least for the waxy oils studied, it is unnecessary to heat the whole bottle before taking each sample, provided that the waxy oil in the bottle is previously homogenized. Cawkwell and Charles [51] have also obtained representative samples of waxy crude oils from steel drums by simply homogenizing the drums before taking a sample.

\subsection{3}

\section{Cooling process}

The third and last step of the sample preparation is the cooling process. It is performed right after the isothermal holding time and consists of the time period in which the sample is cooled from the starting temperature, $T_{i}$, to the 
measurement temperature, $T_{0}$. In our case, we cool the sample performing a cooling ramp in which a constant cooling rate, $\dot{T}_{h}$, and a constant shear rate, $\dot{\gamma}_{h}$, are used. The subscript " $h$ " stands for "history." The cooling process is carried out to ensure that measurements are performed under controlled shear and thermal histories at the measurement temperature $T_{0}$.

The cooling process must be performed with the appropriate geometry, gap, and temperature range for the waxy oil at hand. It is clear that the same geometry and gap employed in the cooling process are also employed in the subsequent rheological measurement. The methods to determine the appropriate test conditions are described in detail in the following section.

\section{4}

\section{Determining the test conditions: the thermal cycle test}

In this section, we describe the thermal cycle test and show with several examples how to use it to determine the appropriate conditions under which rheological measurements of a given waxy crude are to be performed to ensure repetitive and reliable data. Each curve presented below is an average of at least three runs, except the ones presented in Figs. 2.7 and 2.9.

\subsection{1}

\section{Description of the thermal cycle test}

The thermal cycle test begins by cooling the sample, at a constant rate $\dot{T}_{h}$, from the starting temperature $T_{i}$ to the measurement temperature $T_{0}$, while shearing it at a constant rate $\dot{\gamma}_{h}$. At the moment when $T_{0}$ is achieved, the temperature ramp is reverted, so that the sample is heated back to $T_{i}$ at the same rate $\dot{T}_{h}$, while keeping the shear rate at $\dot{\gamma}_{h}$. The viscosity is plotted as a function of temperature to unveil important properties of the crude. The $\eta \times T$ result of a typical thermal cycle test is given in Fig. 2.1(a).

\subsection{2}

\section{Characteristic temperatures}

In Fig. 2.1(a), the main parameters that can be determined in the thermal cycle test are indicated. Starting at $T_{i}$ and following the cycle path indicated by the arrows in this figure, it is seen that initially the viscosity increases mildly with the temperature up to the crystallization temperature $T_{c}$, displaying the typical Arrhenius temperature dependence of Newtonian fluids. In fact, it is reported in the literature that a Newtonian behavior is observed in this temperature range $[53,31,57]$. Beyond (below) $T_{c}$, a dramatic increase in viscosity takes place, because of the fact that a significant amount 
(a)

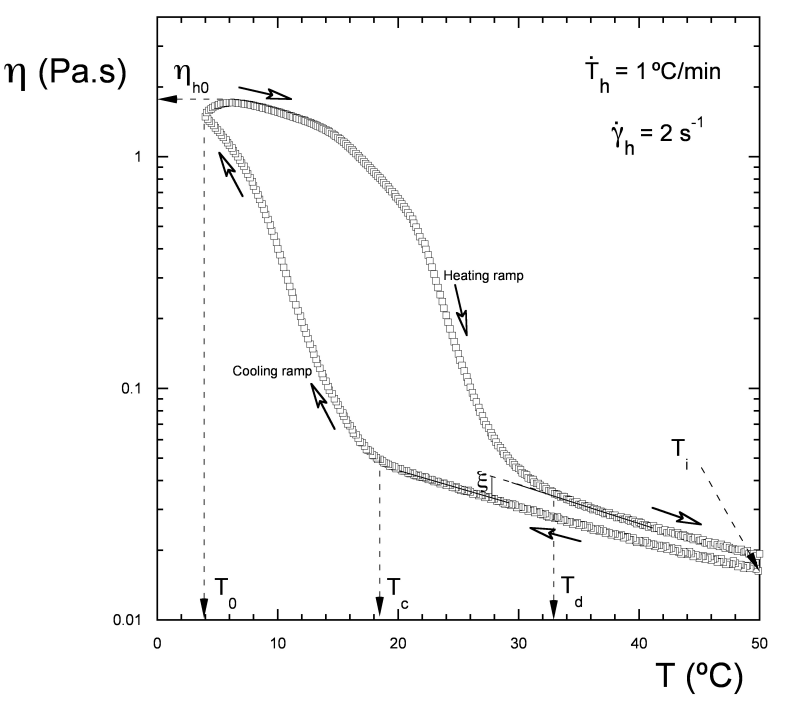

(b)

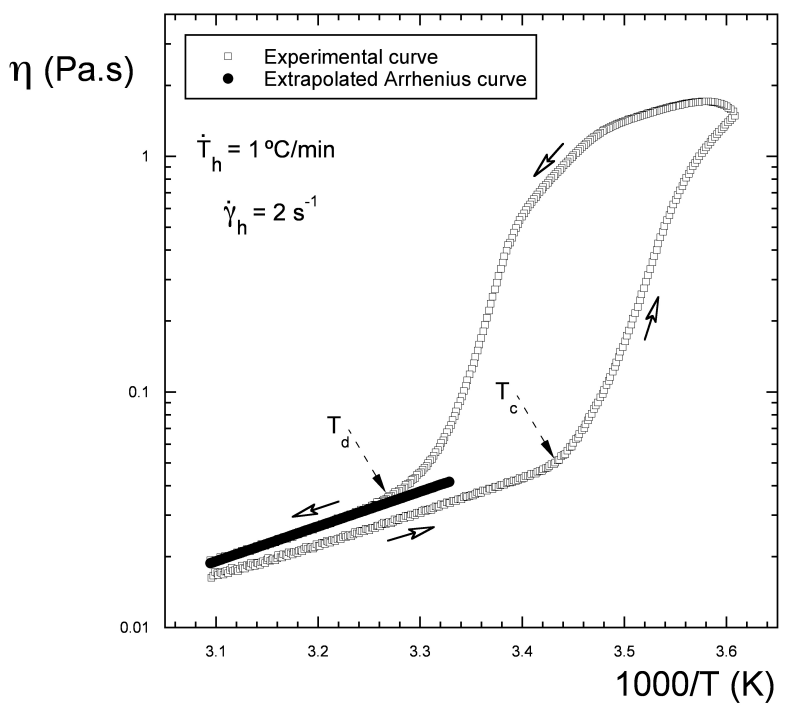

Figure 2.1: A thermal cycle test viscosity plot, with the definitions of $T_{c}, T_{d}$, $T_{i}, T_{0}, \eta_{h 0}$, and $\xi$ : (a) normal plot, and (b) inverse absolute temperature scale plot.

of wax crystals increasingly precipitates and starts to interact. According to Rønningsen et al. [57], a gel-like structure forms at some temperature (not indicated in Fig. 2.1(a)) below $T_{c}$, when approximately $3-4 \%$ of precipitated wax by weight is present.

The crystallization temperature $T_{c}$ is the temperature at which the minimum amount of wax crystals needed for a non-Arrhenius behavior is reached. Such behavior is characterized by a sharp change in the slope of the viscosity-temperature curve (Fig. 2.1(a)). The gel-like structure grows stronger as the temperature decreases further below $T_{c}$, accompanied by corresponding changes in the rheological behavior. The oil becomes more and more nonNewtonian, with a rheological behavior that is a strong function of the shear and thermal histories, because they govern the amount, size, and shape of wax 
crystals. The non-Newtonian behavior of the oil typically includes the viscosity dependence upon the shear rate, time of shear, and temperature.

It is important to emphasize that the WAT, defined as the temperature at which the first wax crystals appear, is not equal to $T_{c}$, because the appearance of the first wax crystals does not necessarily alter the viscosity of the oil [53]. Thus, the WAT is usually higher than $T_{c}$, as shown by Ronningsen et al. [57] for different crudes, and cannot be seen in plots like the plot in Fig. 2.1(a). Unfortunately, it is not uncommon in the flow assurance literature to find the terms $W A T$ and $T_{c}$ being used indistinctly.

Below the WAT, new wax crystals precipitate and the already existing wax crystals grow, as the temperature is decreased. Both the solid fraction and crystal length distribution are then increased, contributing to the build up a structure that is formed by the interaction between wax crystals. When the sample is cooled under shear, there is also a tendency of the microstructure to break down, so that buildup and breakdown of the microstructure occur simultaneously.

In fact, the amount of degradation that the microstructure experiences depends upon not only the shear rate $\dot{\gamma}_{h}$. Indirectly, it also depends upon the cooling rate $\dot{T}_{h}$, because this parameter determines the duration of the cooling ramp and, hence, how long the sample will be under shear. In this way, $\dot{T}_{h}$ is the driving force for microstructure buildup, while microstructure breakdown depends upon the magnitudes of both $\dot{\gamma}_{h}$ and $\dot{T}_{h}$.

The upper portion of the loop shown in Fig. 2.1(a) pertains to the heating part of the thermal cycle test. It is interesting to note that the viscosity reaches its maximum, $\eta_{h 0}$, at a temperature that is slightly above $T_{0}$. This may be because the time scale of the microstructure changes with the temperature is comparable to the time scale of the imposed temperature changes. Past $\eta_{h 0}$, the viscosity decreases as the sample is heated, because the wax crystals start to dissolve.

At a given point along the heating portion of the loop, the dissolution temperature $T_{d}$ is achieved (Fig. 2.1(a)), above which the Arrhenius behavior of the oil is restored. The difference between the dissolution and crystallization temperatures is related to the imposed value of $\dot{T}_{h}$, which is responsible for this apparent thermal hysteresis. Decreasing $\dot{T}_{h}$ causes $T_{c}$ to approach $T_{d}$. In this connection, the quantity $\xi$ in Fig. 2.1(a) characterizes the difference between viscosities of the sample corresponding to the Arrhenius sectors of the cooling and heating portions of the loop. A detailed discussion about this behavior is given later on in this text.

The crystallization and dissolution temperatures $T_{c}$ and $T_{d}$ can also be 
quantified in an inverse absolute temperature scale plot, as indicated in Fig. 2.1(b). This type of plot is used by some authors (e.g. Rønningsen et al. [57] and Webber [31, 58]).

Analogous to the $W A T$ definition, the Wax Disappearance Temperature $(W D T)$ is defined as the temperature at which the last wax crystals disappear. Thus it is clear that the $W D T$ and the dissolution temperature $T_{d}$ are different quantities, in the same way that the $W A T$ and $T_{c}$ also imply different concepts $[53,31]$.

\subsection{3}

\section{Choice of the geometry}

The choice of the geometry to be used in the rheological measurements of a waxy crude oil is of central importance and has been theme of investigation in the last few decades.

Many authors $[33,34,46,3,51,52,53,12,54,6,55]$ used the cone-andplate geometry based on the fact that this is the only geometry that imposes a homogeneous flow (spatially uniform shear rate). Homogeneity is desired to ensure the same shear history to all material elements within the sample, as well as to eliminate shear-rate-gradient-induced particle migration.

A potential disadvantage of the cone-and-plate geometry for the present application resides in the typically small gaps, especially in the vicinity of the cone tip. The presence of wax crystals whose characteristic dimension is of the same order of magnitude of the gaps may introduce measurement errors.

To help with the understanding of the effect of the geometry choice on the measurements, we performed a number of thermal cycle tests, with all of them keeping the same values of $T_{i}, T_{0}, \dot{T}_{h}$, and $\dot{\gamma}_{h}$, but with several geometries, namely, two different cone-and-plate geometries, a smooth Couette geometry, and parallel-plate geometries with different gaps. The results are summarized in 2.2 .

This figure illustrates the strong dependence of the results on the geometry chosen. Different geometries give rise to different results, and the results pertaining to the parallel-plate geometries strongly depend on the gap used. Moreover, this figure illustrates that, in general, small-gap geometries lead to higher values of $T_{c}$ and viscosity.

The results pertaining to the two cone-and-plate geometries are in good agreement, yielding higher viscosity levels and much higher crystallization temperatures, as compared to the results obtained with the parallel-plate geometry with a wide gap $(3 \mathrm{~mm})$. It is also seen that high values of both $T_{c}$ and $\eta_{h 0}$ are also obtained with the parallel-plate geometry with the smaller 


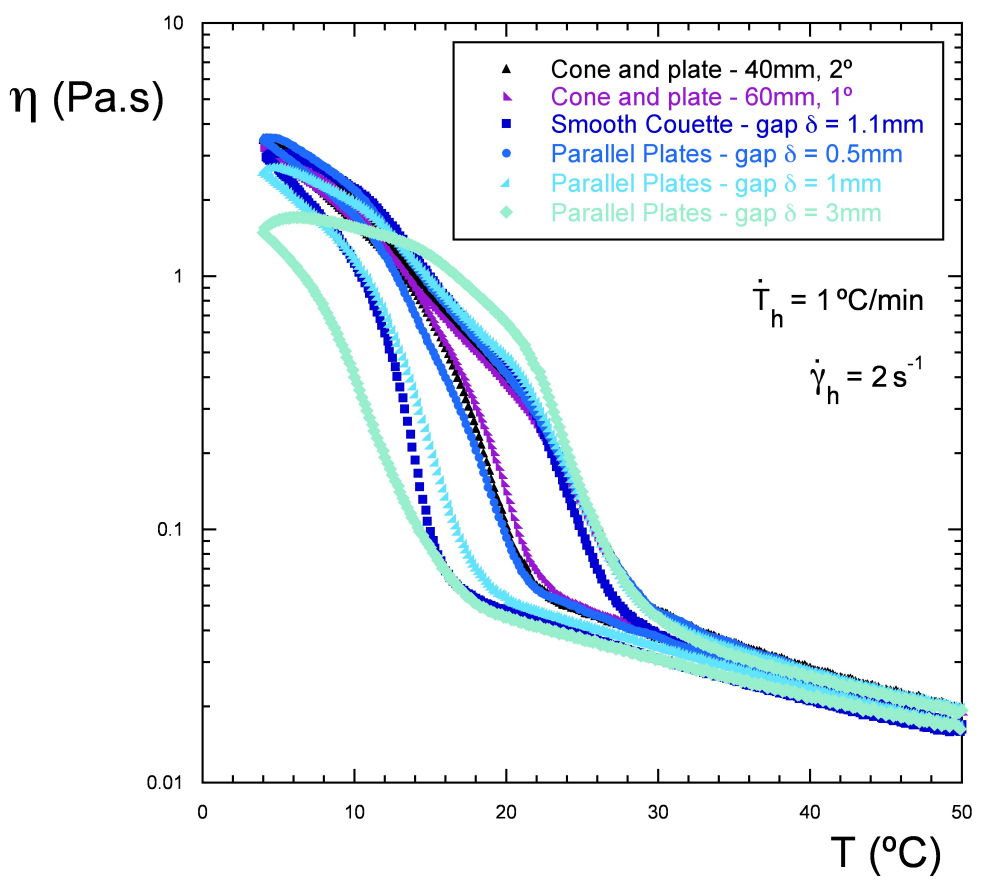

Figure 2.2: Thermal cycle test results for different geometries.

gap. That is, as the gap is decreased, the measured values of both $T_{c}$ and $\eta_{h 0}$ increase. In fact, the curve in Fig. 2.2 pertaining to the $500 \mu \mathrm{m}$ gap parallelplate geometry is in good agreement with the curves for the two cone-and-plate geometries.

We are thus in a position to decide which results are more reliable, the results pertaining to the parallel-plate geometry with wide gaps or the results obtained with the cone-and-plate geometries and also with the smallgap parallel plate geometry. In the remainder of this section, we provide the set of evidences and arguments that led us to believe that, at least for the waxy crude investigated, the results obtained with the wide-gap parallel-plate geometry are more reliable.

First, it is important to emphasize that, when small-gap geometries are employed, the presence of wax crystals may violate the continuum hypothesis assumed in the rheometer theory. When the gap is of the same order of magnitude as the typical size of the wax crystals or aggregates, the continuum hypothesis is known to be inappropriate, and thus, measurement errors are expected. In favor of the cone-and-plate geometry, it is often argued that the typical radii in the small-gap region are also small, and thus, the contribution of this effect to the total torque should be negligible. However, aggregates of sizes as large as $0.5 \mathrm{~mm}$ have been reported [31] for some waxy oils. Such sizes would require a minimum gap much wider than the maximum gap found in most cone-and-plate geometries. Moreover, as far as the present authors know, 
no systematic study of the importance of this potential source of experimental error is available in the literature.

Another evidence that indicates that the small-gap geometries give too high values for $T_{c}$ and $\eta_{h 0}$ comes from the microscopic measurements performed by Rønningsen et al. [57], who observed that decreasing the oil film thickness causes the WAT to increase. Small gaps seem to promote earlier (i.e. at higher temperature) precipitation of wax crystals because of some nucleation mechanism, disturbing the natural bulk crystallization process and resulting in high values of $T_{c}$ as well. Moreover, because the wax crystals are formed earlier, larger sizes and concentrations of crystals can occur at a given temperature, resulting in higher viscosity levels.

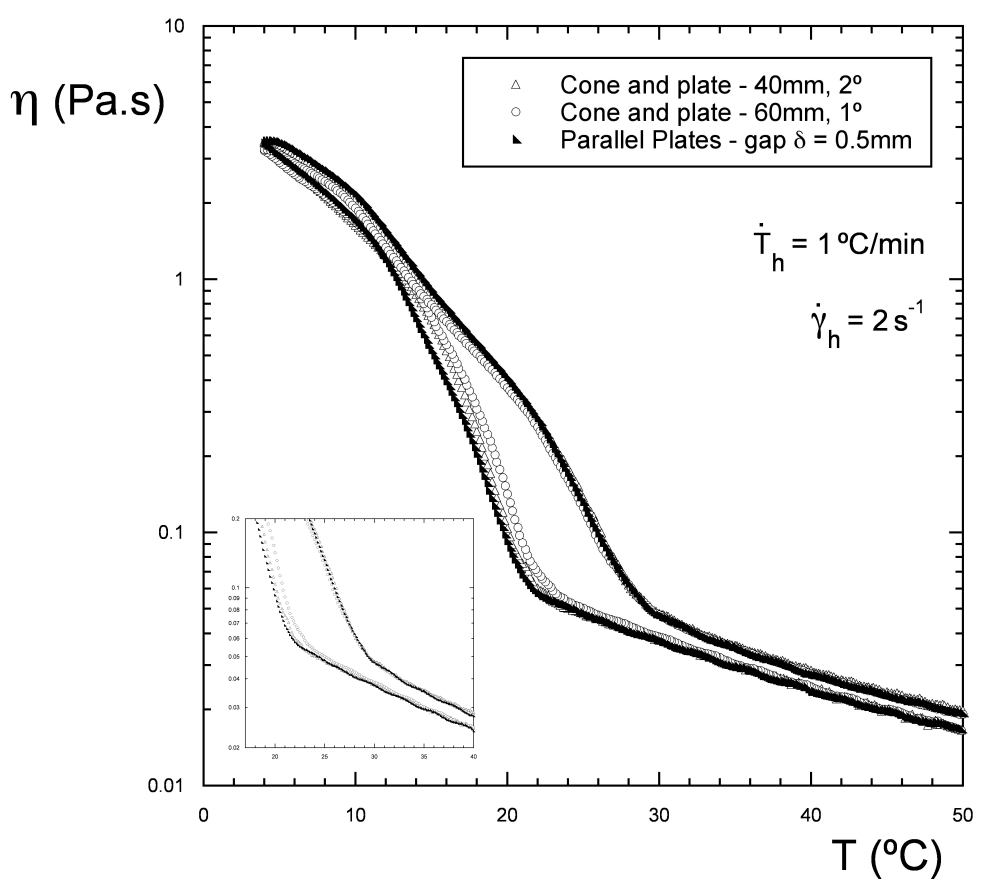

Figure 2.3: Thermal cycle test results for three different small-gap geometries.

The two cone-and-plate geometry results and the $0.5 \mathrm{~mm}$ gap parallelplate geometry results are replotted in Fig. 2.3, to allow for a more detailed comparison. No significant differences are observed in the $\eta_{h 0}$ and $T_{c}$ values obtained with the three geometries, except for the $T_{c}$ value given by the $60 \mathrm{~mm}$ diameter cone-and-plate geometry, which is slightly higher. This suggests that the inhomogeneity that is present in the parallel-plate geometry has no important impact on the measured values, at least in this gap range and for the waxy oil under investigation. Further evidence that points toward the small importance of flow inhomogeneity, for a wider gap, is given in the subsection entitled "Systematic errors" below. 
The temperature control may be a problem when the parallel-plate geometry is employed with too wide gaps, because the rheometer temperature is imposed in one of the plates only, and hence, large sample volumes may lead to important temperature inhomogeneities. In other words, the actual average temperature of the sample may differ significantly from the imposed temperature. This problem is potentially important when the imposed and laboratory temperatures are too far apart only. The actual average temperature is higher than the imposed temperature when the latter is lower than the laboratory temperature and vice versa. Now turning to Fig. 2.2, it is seen that, coincidentally for this oil, all of the $T_{c}$ values obtained with the different geometries range around the laboratory temperature $\left(\approx 23^{\circ} \mathrm{C}\right)$, and hence, it is fair to expect that the $T_{c}$ values obtained are not affected significantly by the temperature control limitations described above. With regard to the $\eta_{h 0}$ values, which correspond to temperatures significantly below the laboratory temperature, it may be argued that the wide-gap $\eta_{h 0}$ value is lower than the others in Fig. 2.2 because of a poor temperature control. In the following section, we show that this does not seem to be the case.

\subsection{4 \\ Influence of the gap}

Once the parallel-plate geometry is elected, it is important to investigate the gap effects on rheological measurements. To this end, a number of thermal cycle tests were performed by keeping $\dot{T}_{h}=1{ }^{\circ} \mathrm{C} / \mathrm{min}$ and varying the gap $\delta$. The results for three different values of $\dot{\gamma}_{h}$ are presented in Fig. 2.4.

The smallest gap $(\delta=500 \mu \mathrm{m})$ leads to the highest maximum viscosity $\eta_{h 0}$ for all cases. In addition, as the gap is increased, both $\eta_{h 0}$ and $T_{c}$ decrease. However, above a certain gap value, no further dependence upon the gap is observed. For example, in Fig. 2.4(a) for $\dot{\gamma}_{h}=2 \mathrm{~s}^{-1}$, we see that, with a gap of $2000 \mu \mathrm{m}$, we obtain approximately the same result as with gaps of $2500 \mu \mathrm{m}$ and $3000 \mu \mathrm{m}$, which indicates that a gap of $2000 \mu \mathrm{m}$ is wide enough in this case.

For shear rates of $20 \mathrm{~s}^{-1}$ and $200 \mathrm{~s}^{-1}$ (panels (b) and (c) of Fig. 2.4), gaps of $1500 \mu \mathrm{m}$ and of $1000 \mu \mathrm{m}$, respectively, are wide enough to ensure gapindependent results. This trend is readily explained by the fact that higher shear rates tend to reduce the size of the crystals and aggregates.

Figure 2.5 further illustrates that, for each combination of parameters, a different minimum gap is needed to ensure gap-independent results.

It is worth noting that the time duration of the thermal cycle test is longer for smaller values of $\dot{T}_{h}$, because the end temperatures are fixed. Therefore, the 
(a)
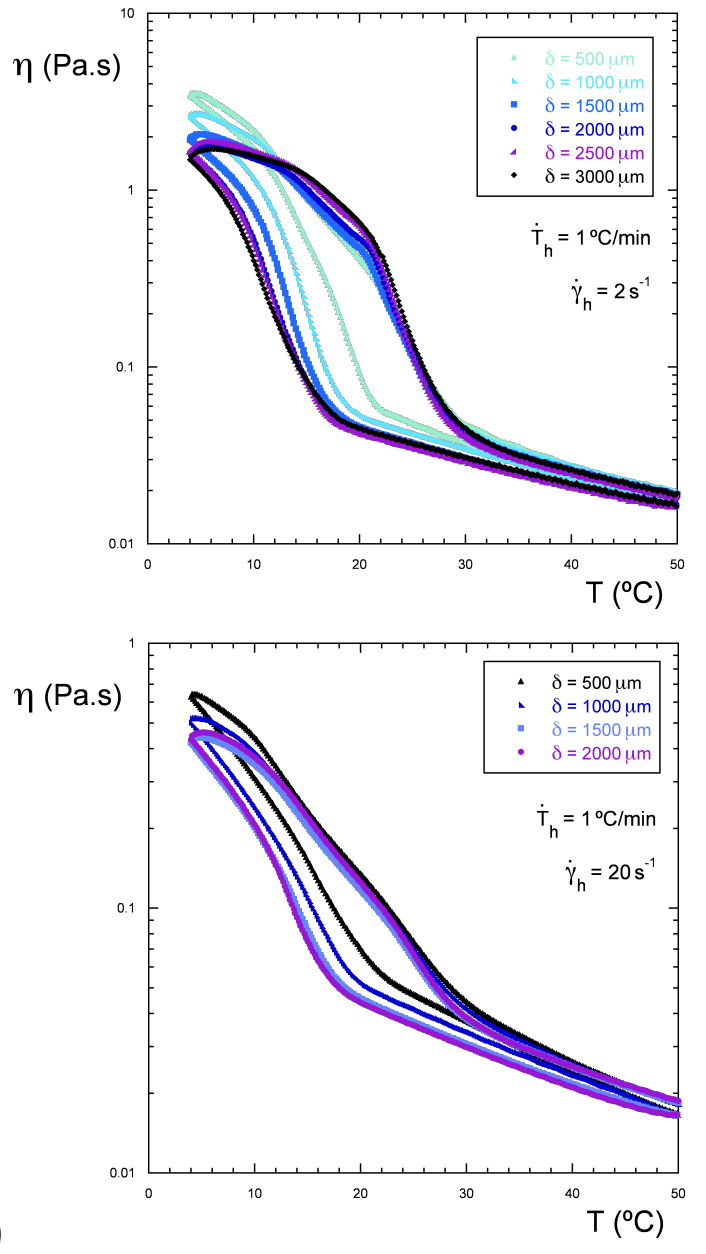

(b)

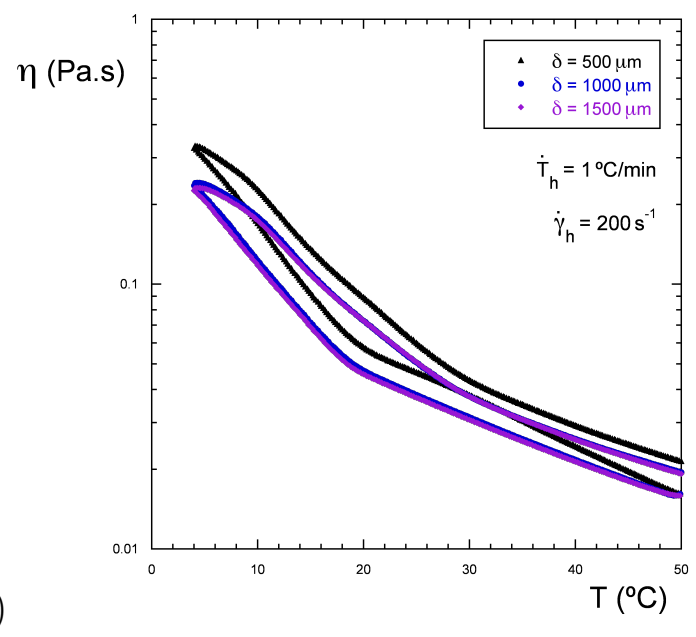

Figure 2.4: Effect of the gap on the crystallization process and rheology: (a) $\dot{\gamma}_{h}=2 s^{-1}$; (b) $\dot{\gamma}_{h}=20 s^{-1}$; (c) $\dot{\gamma}_{h}=200 s^{-1}$. 


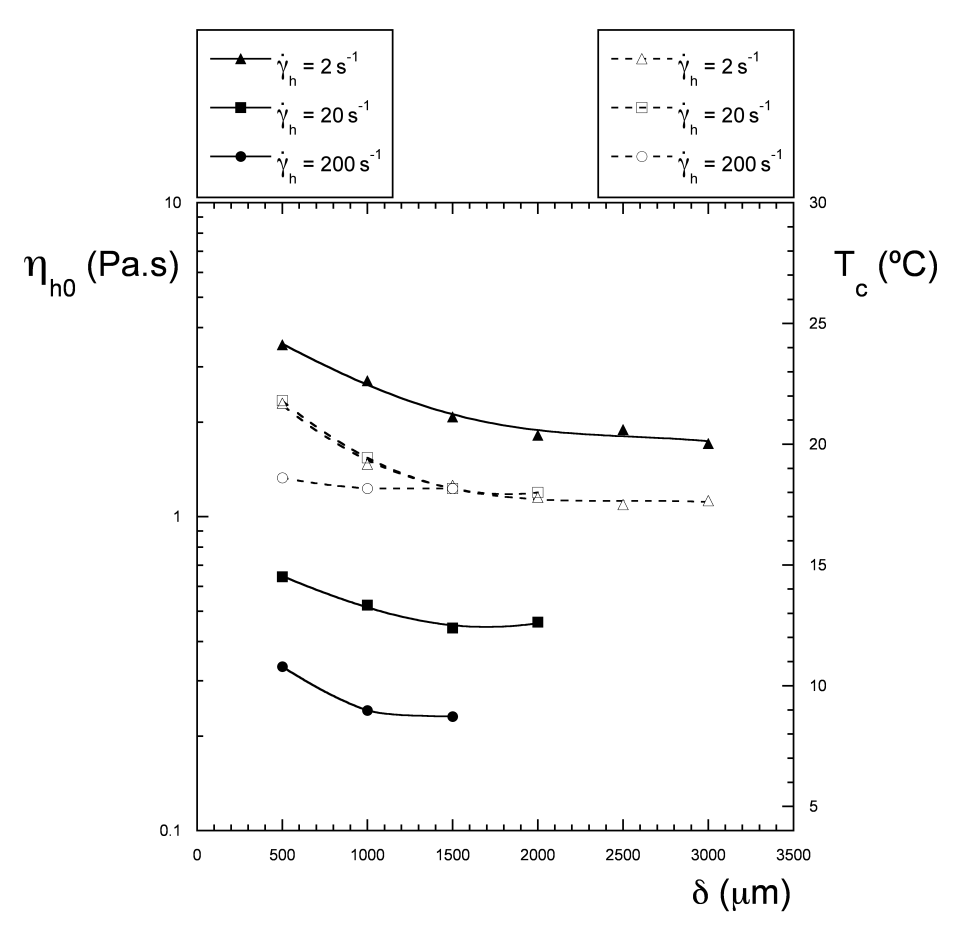

Figure 2.5: Maximum viscosity $\eta_{h 0}$ and crystallization temperature $T_{c}$ as a function of the gap.

sample is subjected to longer periods of shearing when $\dot{T}_{h}$ is smaller, and thus, more degradation of the microstructure is expected. On the other hand, lower values of $\dot{T}_{h}$ favor the appearance of larger wax crystals and/or aggregates, and hence, wider gaps are needed to ensure gap-independent results. Therefore, the minimum gap required to obtain gap-independent results depends upon both $\dot{T}_{h}$ and $\dot{\gamma}_{h}$.

In fact, with these results, it is possible to estimate the characteristic length of the microstructure components (crystals or aggregates) for each fixed shear and thermal history - i.e. a fixed pair $\left(\dot{T}_{h}, \dot{\gamma}_{h}\right)$. To this end, we rely on the rule of thumb that dictates that the gap should be more than ten times the particle size to produce gap-independent results [59]. The estimated characteristic sizes of the microstructure components for $\dot{T}_{h}=1{ }^{\circ} \mathrm{C} / \mathrm{min}$ are $200 \mu \mathrm{m}$ for $\dot{\gamma}_{h}=2 \mathrm{~s}^{-1}, 150 \mu \mathrm{m}$ for $\dot{\gamma}_{h}=20 \mathrm{~s}^{-1}$, and $100 \mu \mathrm{m}$ for $\dot{\gamma}_{h}=200 \mathrm{~s}^{-1}$.

There are three different morphologies reported in the literature for wax crystals, namely, needles, plates, and malformed crystals. While malformed crystals are often smaller, needles can achieve the lengths estimated by the above analysis $[31,60]$. Nevertheless, because the microscope was not yet brought into play, at this time, we cannot provide further evidence about the validity of the above estimate, and hence, there is no grounds to draw definitive conclusions about the morphology of the crystals.

The fact that gap-independent results are obtained at sufficient wide gaps 
not only can be explained but also supports the hypothesis that the smallgap geometries yield wrong results because of the violation of the continuum hypothesis. Moreover, it constitutes strong evidence that thermal control is not a problem in the rheological measurements performed with the parallel-plate geometry, for the Brazilian waxy crude tested.

Above, we presented experimental evidence in support of the usage of parallel-plate geometries with wide enough gaps. However, additional research work is still needed to verify the importance of the flow inhomogeneity of this geometry on rheological measurements. To this end, a systematic investigation using parallel-plate geometries with different diameters is in order.

\subsection{5}

\section{Choice of the starting temperature $T_{i}$}

In our specific case, we are dealing with the design of a subsea pipeline to transport a waxy crude oil along the sea floor in a deepwater environment. The oil is expected to enter the line at a temperature not higher than $60^{\circ} \mathrm{C}$, and the main concern is wether or not the pipeline will restart after a long period of shutdown. Our measurement temperature $T_{0}$ was fixed at $4{ }^{\circ} \mathrm{C}$, because this is the expected temperature on the sea floor.

The choice of the starting temperature $T_{i}$ of the thermal cycle test is quite elaborate. Effects of resins and asphaltenes on wax crystallization render the crystallization temperature $T_{c}$ dependent upon the starting temperature $T_{i}$. For our application, it is important to choose the value of $T_{i}$ that yields the highest possible crystallization temperature $T_{c}$, because it corresponds to the worst possible condition for the startup problem.

A detailed discussion about the effects of resins and asphaltenes on wax crystallization and also on rheological properties is given by Smith and Ramsden [1]. They employed an experimental procedure to determine the starting temperature that yields the highest possible "gelling temperature". According to these authors, "...the maximum pour point is obtained when the reheat temperature is high enough to dissolve the wax, but not high enough to dissolve or disassociate the resins sheaths around the wax crystals."

Thus, as explained by these authors, cooling the oil sample again from the starting temperature induces the wax to reprecipitate, but the resin molecules are not free to associate with the newly precipitated wax, leading to a high pour point. If a higher starting temperature is used, then the resins start to dissolve and become free to act as natural pour-point depressants, collecting around the wax crystals and preventing the formation of the interlocking network between them [1]. The same line of reasoning can be applied to understand 
what happens with the oil if a more vigorous pretreatment is chosen.

Rønningsen et al. [57] observed the same general behavior regarding the action of resins and asphaltenes described by Smith and Ramsden [1] and also emphasized the importance of controlling the prior thermal treatment (the pretreatment) applied to the oil to obtain meaningful rheological data. In addition, Rønningsen et al. [57] showed that the action of natural pour-point depressants is to promote the formation of large crystal aggregates. According to them, the flow-improving effect is manly due to the organization of needles and plates into weakly interacting spherulitic structures. It is interesting to note that Venkatesan et al. [6] observed that the addition of asphaltenes to waxy-oil mixtures reduces the yield stress by leading to the formation of smaller crystals.

Successive (preliminary) thermal cycle tests can also be used to determine the starting temperature that yields the maximum crystallization temperature in analogy to the procedure employed by Smith and Ramsden [1]. Keeping in mind the startup problem, a possible procedure is:

(i) to keep a certain volume of the crude oil at $60^{\circ} \mathrm{C}$ for a long enough time, then to stir it vigorously to achieve homogenization, and to split the volume of oil into several bottles;

(ii) to cool the bottles suddenly to $4^{\circ} \mathrm{C}$, to precipitate tiny wax crystals with associated resins and asphaltenes, and to keep them stored at $4{ }^{\circ} \mathrm{C}$;

(iii) to reheat one of the bottles to $60^{\circ} \mathrm{C}$ and to perform a thermal cycle test to determine the crystallization temperature pertaining to a starting temperature of $60^{\circ} \mathrm{C}$;

(iv) to reheat another bottle to another starting temperature that is below $60{ }^{\circ} \mathrm{C}$ but above the just obtained crystallization temperature and to perform another thermal cycle test to determine the crystallization temperature pertaining to the new starting temperature;

(v) to repeat step (iv) for several starting temperatures, to determine the crystallization temperature as a function of the starting temperature;

(vi) to observe which starting temperature yields the maximum crystallization temperature. If it is observed that $T_{c}$ stops varying above a certain value of starting temperature, then choose the lowest possible starting temperature that yields the maximum $T_{c}$. This is intended to minimize sample contraction and irreversible changes of the oil due to hightemperature exposure. Otherwise, choose the starting temperature that 
yields the maximum $T_{c}$ as the starting temperature $T_{i}$ to be used in the rheological measurements.

In the present research, we employed the just described procedure, except that, because of limitations of our laboratory facilities, we stored the bottles at ambient temperature $\left(\simeq 23^{\circ} \mathrm{C}\right)$.

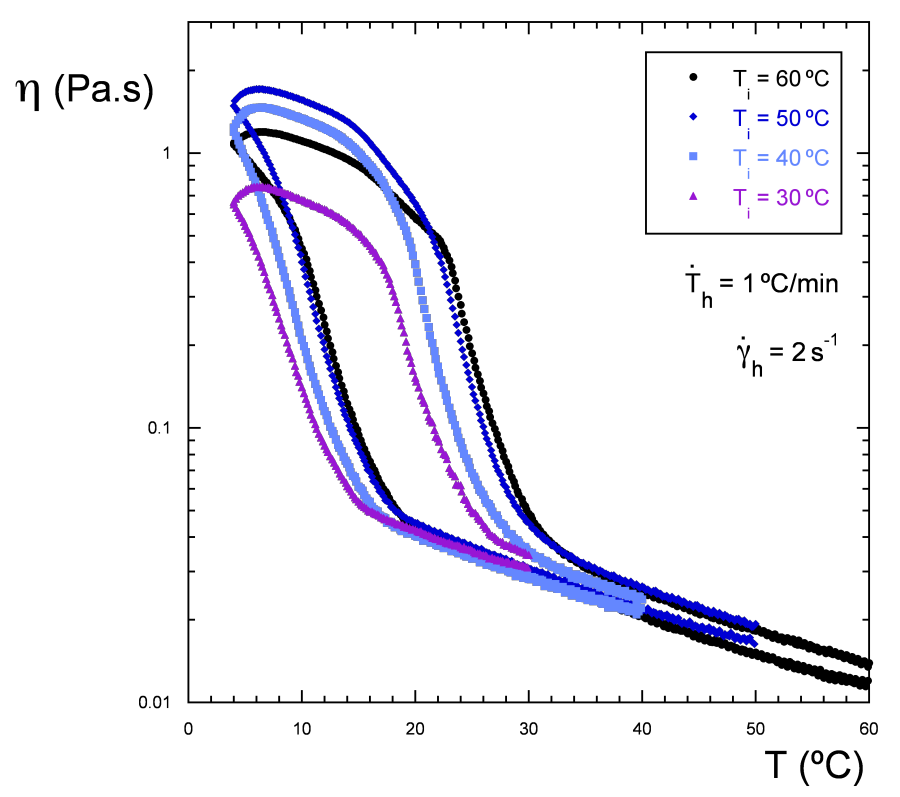

Figure 2.6: Effect of $T_{i}$ on the crystallization process and rheology.

The results of thermal cycle tests performed at four different starting temperatures $T_{i}$ are shown in Fig. 2.6. This figure illustrates that increasing $T_{i}$ promotes an increase in $T_{c}, T_{d}$, and $\eta_{h 0}$ until $T_{i}$ is equal to $50^{\circ} \mathrm{C}$. Above this starting temperature, no changes in $T_{c}$ and $T_{d}$ are observed, indicating that, above this limit, the crystallization and dissolution processes are no longer affected by the value chosen for $T_{i}$. This thermal memory that is observed at lower starting temperatures is due to unmelted nuclei or nucleus fragments.

Thus, for our purposes, $50^{\circ} \mathrm{C}$ seems to be an appropriate choice for the starting temperature, because it represents the lowest temperature within the range of the industrial process that yields the maximum measured crystallization temperature. Because the bottles were not stored at the measurement temperature, this choice for $T_{i}$ may not yield the highest possible crystallization temperature for this oil. As discussed above, the storage temperature should, in principle, be equal to the measurement temperature. However, if all of the resins and asphaltenes are already precipitated at some limiting temperature that is higher, then it should be of no consequence to store the oil at any temperature up to this limiting temperature. We cannot quantify the effect of having stored our oil at $23^{\circ} \mathrm{C}$, because we do not know this limiting temperature, but we estimate that at least most of these fractions are no 
longer dissolved or dissociated at $23^{\circ} \mathrm{C}$, because of the proximity between this temperature and the crystallization temperature $T_{c}$. Nevertheless, the choice of $50^{\circ} \mathrm{C}$ for $T_{i}$ seemed to be the best choice at hand, given our laboratory limitations.

It is important noting that, in the above-described procedure, it is assumed that, in general, resins and asphaltenes dissolve or dissociate at high enough temperatures. This assumption was previously made, as discussed in detail by Smith and Ramsden [1], and seems to explain nicely their results.

In Fig. 2.6, it is seen that the maximum viscosities obtained for $50^{\circ} \mathrm{C}$ and $60^{\circ} \mathrm{C}$ do not coincide, as it might be expected. A possible explanation for this difference is the presence of resins and asphaltenes in the crude oil that would start to dissolve or dissociate at higher temperatures only. Thus, above $60^{\circ} \mathrm{C}$, the dissolution or dissociation of these fractions is more pronounced, causing the resins and asphaltenes to act as natural pour-point depressants during cooling, as discussed above. At $60^{\circ} \mathrm{C}$, the amount of resins and asphaltenes that is dissolved may not be large enough to reduce $T_{c}$ and $T_{d}$, but is large enough to diminish the value of the maximum viscosity.

The choice of $50^{\circ} \mathrm{C}$ is also supported by the fact that this turned out to be the starting temperature that gives the highest maximum viscosity. Thus, it is a conservative choice as far as the startup problem is concerned.

The curves starting at $30^{\circ} \mathrm{C}$ and $40^{\circ} \mathrm{C}$ in Fig. 2.6 display lower values of $T_{c}$, because at these starting temperatures, the wax crystals do not dissolve completely. This partial dissolution is enough to restore the Arrhenius behavior, by breaking the interlocking network between crystals. However, nonnegligible amounts of high-molecular-weight wax still remain suspended as crystals in the oil. Consequently, during cooling, the lower-molecular weight wax recrystallize and form again an interlocking network but a weaker one, giving rise to lower values of $T_{c}, T_{d}$, and $\eta_{h 0}$. These effects are related to the previously mentioned thermal memory of the oil.

The effect of the starting temperature on $T_{c}, T_{d}$, and $\eta_{h 0}$ can be used to estimate the WAT, as explained next. If the starting temperature is high enough to dissolve the waxes but not high enough to dissolve the resins and asphaltenes, then no wax will remain undissolved at the beginning of the thermal cycle and there will be no natural pour-point depressants to affect the parameters $T_{c}, T_{d}$, and $\eta_{h 0}$. For the oil studied in the present research, the WAT is estimated to be between $40^{\circ} \mathrm{C}$ and $50^{\circ} \mathrm{C}$, perhaps closer to $50^{\circ} \mathrm{C}$, because this is the range within which the parameters stop varying. 


\section{5}

\section{Reliability and sources of error}

\subsection{1}

\section{Repeatability}

(a)
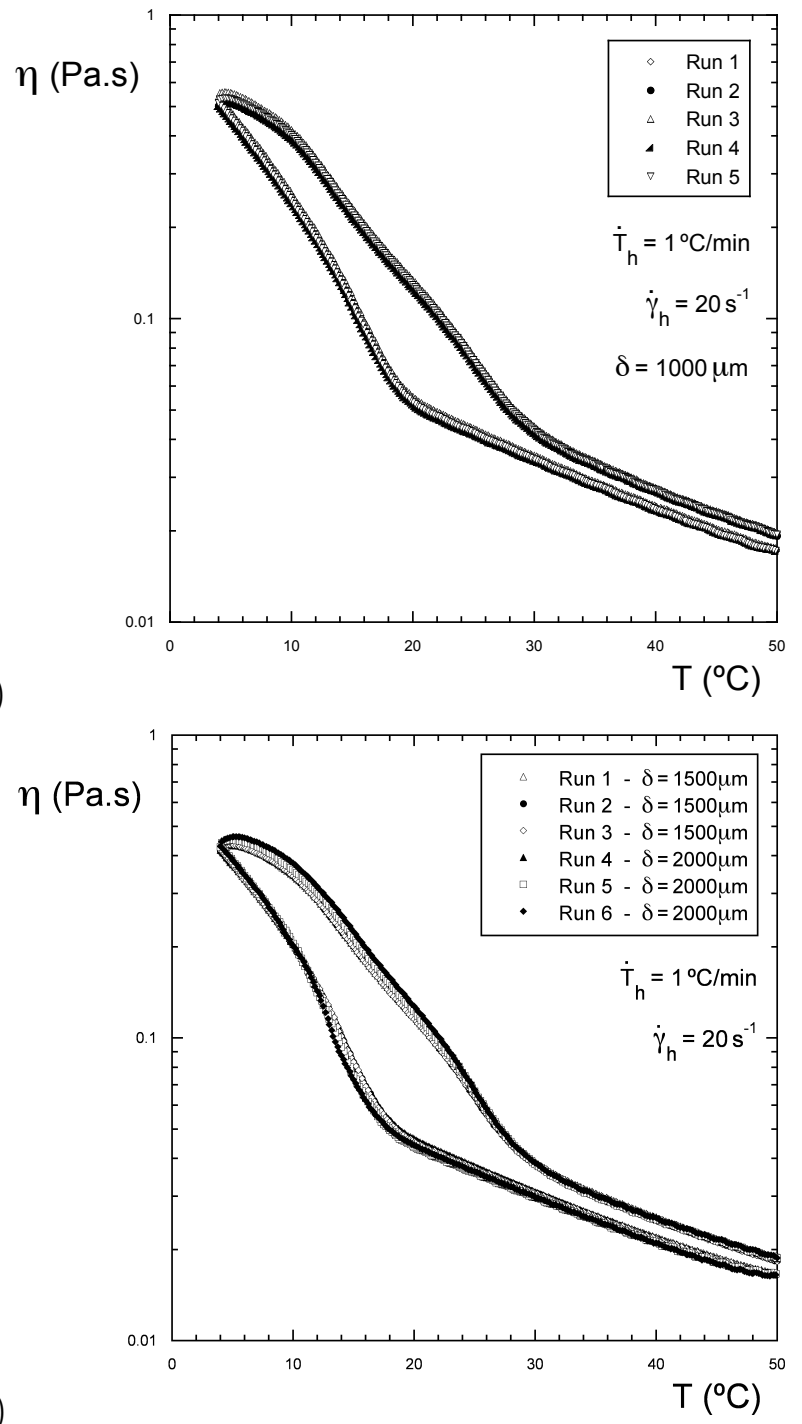

Figure 2.7: Temperature cycle - repeatability and reliability.

The repeatability of the methodology was verified by performing the same thermal cycle test for several times and comparing the results obtained. A fresh sample was used in each repetition. The results of such tests for a representative pair of values of $\dot{T}_{h}$ and $\dot{\gamma}_{h}$ are presented in Fig. 2.7. It can be seen in this figure that the curves pertaining to all the repetitions are essentially coincident, demonstrating the repeatability of the methodology.

The gap used in the tests pertaining to Fig. 2.7(a) was equal to $1000 \mu \mathrm{m}$, which is not wide enough for the applied shear rate of $20 \mathrm{~s}^{-1}$, as discussed above. In fact, we showed previously that, for this rate, the minimum gap 
should be equal to $1500 \mu \mathrm{m}$ to ensure gap-independent results. This fact illustrates that repeatability does not necessarily imply accuracy or correctness.

In Fig. 2.7(b), six different curves are plotted, pertaining to three repetitions for each of the two different gaps employed, both in the correct range. Therefore, the results in this figure are gap-independent and possess repeatability.

\subsection{2}

\section{Systematic errors}

To check for possible systematic errors, results obtained with two different rheometers were compared. The instruments employed were the MCR-501 (Anton Paar) with $50 \mathrm{~mm}$ diameter smooth parallel plates and the AR-G2 (TA Instruments) with $60 \mathrm{~mm}$ diameter smooth parallel plates.

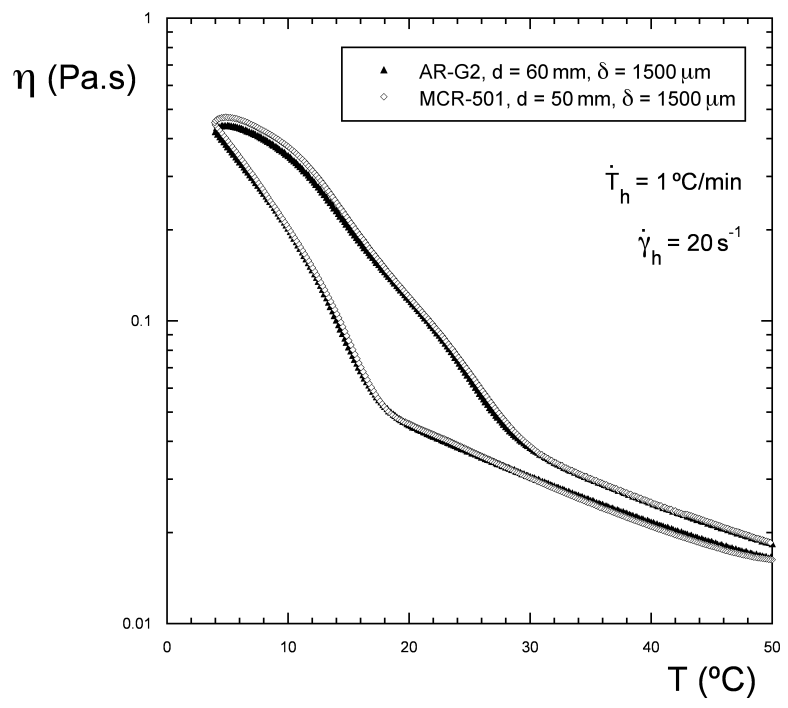

Figure 2.8: Comparison between data obtained with two different rheometers.

Figure 2.8 demonstrates that the results obtained do not significantly depend upon the instrument employed. The small difference between the curves in the low-temperature region is due to differences in the performance of the thermal control systems of the instruments.

It is interesting to note that the same gap was used in both geometries, but as specified above, the diameters of the parallel-plate geometries were different. The agreement between these results not only show that systematic errors are not relevant, but also is an evidence that inhomogeneous flow does not impact our measurements significantly. 


\subsection{3}

\section{Chemical stability}

Another potential source of measurement error is related to irreversible changes in the chemical composition and/or microstructure of the waxy oil during the rheological measurement. In fact, this source of error is responsible for the noncoinciding Newtonian portions of the thermal-cycle-test curves, observed in all thermal-cycle-test results presented in this thesis and characterized by the quantity $\xi$ defined in Fig. 2.1(a). The occurrence of a nonzero (positive) $\xi$ is due to irreversible changes in composition or microstructure of the oil during the thermal cycle test. Webber [31] obtained $\xi=0$ for lubricating mineral oils, probably because these fluids possess a much smaller range of chemical compounds when compared to our crude oil.

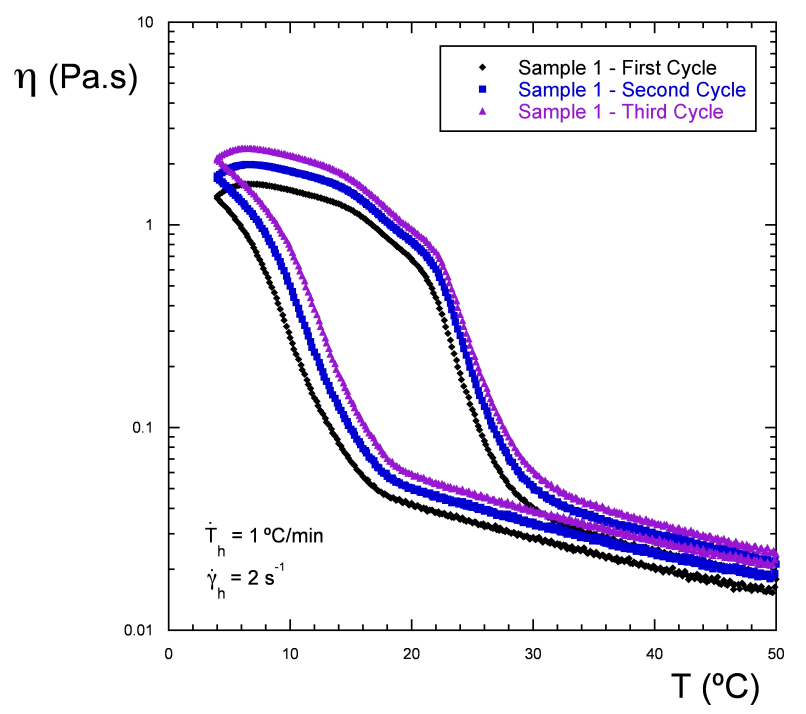

Figure 2.9: Results of the triple thermal cycle test.

To investigate this error source, triple thermal cycle tests were performed. This test consists simply of performing three thermal cycle tests consecutively with the same sample.

Figure 2.9 shows the results of a triple thermal cycle test pertaining to $\dot{T}_{h}=1^{\circ} \mathrm{C} / \mathrm{min}$ and $\dot{\gamma}_{h}=2 \mathrm{~s}^{-1}$. It can be seen that a curve pertaining to a later cycle always appears above the curves pertaining to earlier cycles, in the same fashion that the end of each curve appears above its own beginning. Moreover, the end of one curve coincides with the beginning of the following curve, as expected.

The difference between successive curves diminishes from one cycle to the next, but they do not disappear. It is worth emphasizing that the microscopic changes that are responsible for this behavior are not due to evaporation of light ends, whose possibility of occurrence was suppressed by previous 
evaporation during the pretreatment. Moreover, a cover was used at all times as an additional precaution.

This behavior is probably due to the occurrence of gradual dissolution and reprecipitation of wax in the presence of resins and asphaltenes, which act on the crystallization process and, therefore, modify the microstructure from one cycle to another.

This effect could be avoided by employing a "harder" pretreatment or a higher starting temperature. However, as already discussed, if, on the one hand, a higher starting temperature could increase the stability of the sample, on the other hand, it could also severely alter the composition and behavior of the oil, which is undesirable because the test conditions would depart from the temperatures found in the field application [1]. In our case, the chemical stability problem is not severe (i.e. $\xi$ is not too large), and hence, we find it more appropriate, to preserve the field conditions and to keep this error source under control, to use a new sample at the beginning of each test.

\subsection{4}

\section{Apparent wall slip and adhesive failure}

In dispersions that possess a yield stress, apparent wall slip occurs because of the formation at the wall of a very thin layer, where only the continuous phase is present (the "depleted" layer). At stresses below the yield stress, the viscosity of the continuous phase is much lower than the dispersion viscosity away from the wall, and then, the depleted layer acts as a lubricating film that causes the impression that the unyielded dispersion is sliding on the solid surface. At higher shear rates (when the stress is above the yield stress), apparent slip is no longer observed.

The main ingredients for the occurrence of apparent wall slip in rheological measurements are [61]: (i) structured materials with large particles or flocs in the disperse phase, (ii) smooth walls, (iii) narrow gaps, and (iv) small shear rates (or stresses). Apparent wall slip manifests itself by producing too low measured viscosities that tend to the correct (slip-free) value as the gap is increased. In addition, sudden breaks and unexpected Newtonian plateaus are often seen in the low shear rate region of the flow curves [61].

All the ingredients listed in the previous paragraph may be present in rheological measurements of gelled waxy oils. Specifically, these materials consist of oil suspensions of wax crystals or aggregates, the rheometrical flows may involve narrow gaps and smooth walls, and the low-shear-rate range is usually of interest, especially in the startup flow in pipelines. Therefore, a detailed investigation regarding the presence of apparent wall slip is in order. 


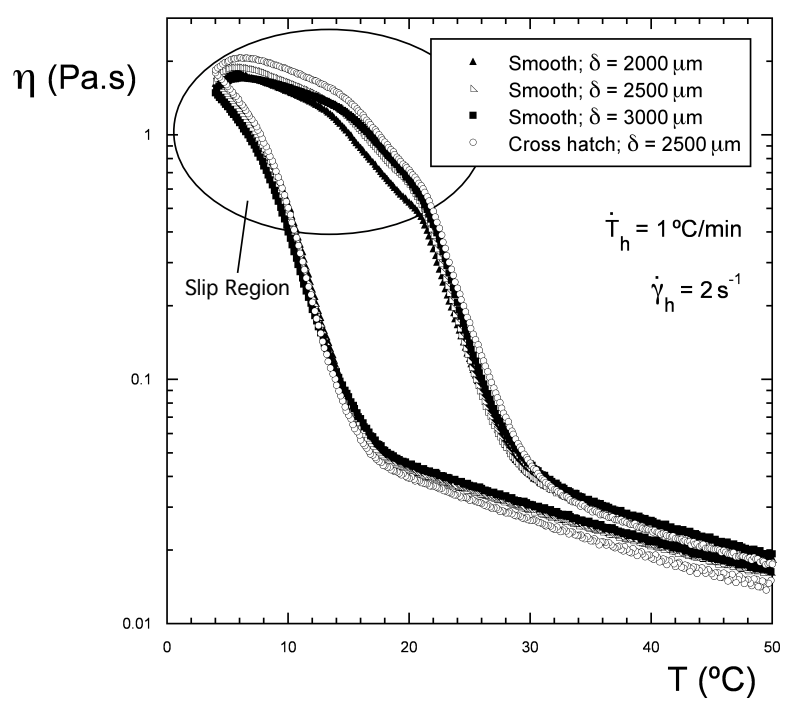

Figure 2.10: Comparison between results obtained with smooth and crosshatched plates.

With regard to thermal cycle tests, apparent slip effects are expected to be observed for small values of $\dot{\gamma}_{h}$ and in the lower temperature portion of the curves. In a given test, it is also expected that these effects are more pronounced as the temperature is decreased, because of the higher solid content. In addition, the occurrence of apparent wall slip is expected to be higher for narrower gaps.

There are two classic ways to deal with apparent wall slip in rheological measurements: (i) to perform tests using different gaps and then obtain slipfree results by extrapolating the data to the limit of very wide gaps [62,61] and (ii) to modify the surface of the rheometer geometry by changing its chemical nature or physical roughness [61]. In the case of changing the chemical nature, a chemical compound that does not have any chemical affinity with the continuous phase must be used as a coating to prevent the formation of the depleted layer. In the case of changing the physical roughness of the surface, a rugosity with characteristic dimension significantly higher than the characteristic sizes of the disperse phase must be used to break the depleted layer and allow for the shear to occur within the bulk of the material.

We performed a set of four thermal cycle tests to illustrate the effects of apparent slip. Figure 2.10 shows the results of these tests. Three of the curves pertain to smooth-parallel plates with three different gaps $(2000 \mu \mathrm{m}$, $2500 \mu \mathrm{m}$, and $3000 \mu \mathrm{m}$ ), while the fourth pertains to cross-hatched plates with a $2500 \mu \mathrm{m}$ gap. The cross-hatched plates are intended to eliminate apparent wall slip, because they present roughened surfaces with a rugosity higher than the characteristic sizes of the microstructure compounds, which is expected 
to break the depleted layer and allow for the shear to occur in the bulk of the gelled oil. It is important to highlight that the use of geometry surfaces with macroscopic rugosities is associated with the hypothesis that no flow is observed between the protrusions and that shear occurs between two imaginary surfaces, each one containing the protrusion tips of each plate. This hypothesis is also used in the vane geometry $[63,64,65]$ and is good when the stresses between protrusions are lower than the yield stress of the gelled oil.

The portion of the curves that is prone to display apparent slip effects corresponds to the low-temperature end of the cycle, as indicated in Fig. 2.10. First, it is noted in this figure that the discrepancies between the curves for different gaps and for cross-hatched plates are not too large, i.e. the apparent slip effects are mild for this specific case, where $\dot{\gamma}_{h}=2 \mathrm{~s}^{-1}$. Rønningsen [3] also observed that apparent wall slip was not serious for crude oils having yield stress lower than about $25 \mathrm{~Pa}$.

For our purpose of illustrating the importance of apparent wall slip, it would have been better to use a lower shear rate, for which more significant apparent slip effects are expected. However, it happens that the viscosity is low at the higher temperatures, and hence, a too low shear rate would imply torques below the resolution of the rheometer sensor, resulting in unreliable data in the high-temperature end of the curves. Nevertheless, the effects observed with $\dot{\gamma}_{h}=2 \mathrm{~s}^{-1}$ seem to suffice for our purposes.

It can be seen from the results in Fig. 2.10 that, in the low-temperature end, the shape of the curves for the two wider gaps are very close to each other, except that the curve for the narrower gap presents a much more pronounced inflection on its heating portion, indicating apparent wall slip. These gapindependent results show that, at this shear rate level, a gap of $2500 \mu \mathrm{m}$ is wide enough to eliminate apparent wall slip. In the Arrhenius portion of the curves (high-temperature end), the viscosities measured with the smooth plates and different gaps are exactly the same, as expected for a Newtonian behavior.

Figure 2.10 also shows the curve for the cross-hatched plates. To analyze the trends of this curve, it is important to keep in mind that, in the rheometer calculations, the gap is taken as the distance between the two parallel imaginary surfaces that are tangent to the protrusion tips. In the high end of the temperature range, it is seen that the viscosities measured with the crosshatched plates are lower than the viscosities measured with the smooth plates, certainly because of the occurrence of flow between protrusions, which work as if there was slip on the just mentioned imaginary surfaces. Actually, this effect is known to render the cross-hatched plates inappropriate for measurements involving Newtonian and other liquids that do not possess a yield stress. 
In contrast to our expectations, in the low end of the temperature range, it is observed in Fig. 2.10 that the curve pertaining to the cross-hatched plates does not agree with the curves pertaining to the smooth plates with wider gaps, yielding higher measured viscosities. It is interesting to note that Wardhaugh and Boger [46] obtained higher yield stress values with roughened surfaces. A possible explanation for this trend is as follows. Because of the heterogeneous nature of the microstructure, the yield surfaces expected to occur at the protrusion tips may not be plane as assumed, leading to a lower effective gap. Nevertheless, it is believed that the gap-independent results obtained with smooth plates are more reliable, because this technique to eliminate slip is thoroughly tested and widely accepted. On the other hand, the usage of crosshatched plates is relatively new, and hence possible error sources remain to be investigated in more detail, such as the influence of the protrusion geometry on the flow pattern.

Recently, Dimitriou et al. [55] have developed a Rheo-PIV system that enables the measurements of local velocity fields. With this system, they were able to observe apparent wall slip directly during rheological measurements with model waxy crude oils.

In another investigation, we performed rheological measurements with a model waxy crude oil formed by a solution of paraffin in kerosene. It is interesting to observe that our experience with this model system is quite different from what we observed with the crude oil, in the sense that significant apparent wall slip was observed. This difference may be related to the presence of polar fractions in crude oils (asphaltenes and resins), that can alter the thickness of the depleted layer and, thus, reduce or eliminate the slip phenomenon. However, further work is needed to better understand the importance of apparent slip in rheological measurements of gelled waxy oils.

It is important to point out that apparent wall slip and adhesive failure are different phenomena. Apparent wall slip is characterized by the existence of a depleted layer of the continuous phase in contact with the geometry surface, and there is no actual slip. Adhesive failure, which, in principle, may also occur during rheological measurements with gelled waxy oils, is a true slip phenomenon, in which the gelled waxy oil sample effectively looses contact with the geometry surface.

In fact, Lee et al. [66] discussed two possible mechanisms in which a gelled waxy oil sample can break, (i) cohesive failure, which can also be understood as yielding, and (ii) adhesive failure. They reported that adhesive failure is more pronounced and usually observed in stress ramps performed with samples cooled at low cooling rates, because under this condition, the samples present 
higher yield stresses (or, in other words, higher cohesive strengths).

Adhesive failure is also undesirable and can be circumvented by increasing the roughness of the surface or changing its chemical composition. A further study to assess the importance of adhesive failure on the rheological characterization of waxy oils is in order.

\section{5 .5}

\section{Other sources of error}

As previously mentioned in the Introduction, Wardhaugh and Boger $[33,34,46]$ discussed in detail the possible sources of error in rheological measurements involving waxy oils. In what follows, we give our assessment regarding the relevance, for the waxy oil studied, of the error sources listed by these authors.

Most rheometers commercially available nowadays are appropriately designed to handle the expansion/contraction of the instrument.

The time lag between the measured and actual sample temperatures also depends upon the rheometer design as well as the type of test. This error can be estimated and corrected by means of heat-transfer calculations and seems to be of minor importance in the present application.

Sample contraction needs attention, and there are two ways of dealing with it: (i) to use a corrected area in the calculations of both the stress and shear rate $[33,34,46]$, and (ii) to use an excess volume to allow for the contraction and, thus, ensure contact with the geometry surface. In the latter case, a small correction is needed for the excess torque generated by the additional volume.

Another possible source of error is related to the deposition of the sample as a non-sheared layer on the instrument surfaces [33, 34, 46, 12]. The occurrence of this problem depends upon the oil composition as well as the temperature range investigated. When it occurs, it is possible to observe, just by visual inspection, a layer of wax deposited on the instrument surfaces. The presence of a non-sheared deposited layer attached to the wall gives rise to a measured viscosity that is higher than the correct one. If deposition is a problem, a Couette geometry with wide gaps and short-duration experiments usually reduce its undesired effects.

Webber [31] discussed the possibility of transient shear-induced heating in the sample at high shear rates as another source of error. We could not observe this effect in our samples in the shear-rate range used. Shear banding is another possible source of measurement error. This phenomenon consists of a flow instability that occurs when the material possesses a nonmonotonic flow 
curve, allowing for multiple shear rate values for a given stress [67, 68]. This may occur in shear flows of gelled waxy oils, because this material may possess nonmonotonic flow curves at low temperatures. Information about this source of error is rather scarce in the literature of waxy oils, and hence, further work is required to better understand its importance.

\section{6}

\section{Final remarks}

In this chapter, we demonstrate that precautions, in addition to the precautions recommended in the literature, must be taken during the preparation of the sample of a waxy crude oil to obtain meaningful rheological data, especially in the case of transient measurements.

We describe a protocol designed to ensure well-defined thermal and shear histories of a waxy crude oil sample to be submitted to rheological measurements. This protocol is subdivided into three steps, namely, the pretreatment, the isothermal holding time, and the cooling process. The pretreatment aims at ensuring a stable chemical composition, through the evaporation of the light ends. The isothermal holding time is applied to redissolve the wax crystals. The cooling process, that is carried out by means of a cooling ramp, with appropriate geometry, gap, and temperature range, imposes well-defined thermal and shear histories to the sample.

The pretreatment conditions should be chosen in such a way not to expose the oil to temperatures that differ from the operating temperature conditions. The usual presence of asphaltenes and resins requires additional precautions.

The thermal cycle test is used to determine test conditions, such as geometry, gap, starting temperature, and other. It consists of cooling the sample in the rheometer at a constant rate $\dot{T}_{h}$, from the starting temperature $\left(T_{i}\right)$ to the measurement temperature $\left(T_{0}\right)$, and then heating it back to $T_{i}$ at the same rate $\dot{T}_{h}$. A shear rate $\dot{\gamma}_{h}$ is applied at all times during the cooling/heating cycle. The viscosity is plotted as a function of temperature to unveil the crystallization and dissolution temperatures ( $T_{c}$ and $T_{d}$, respectively), as well as the maximum viscosity $\eta_{h 0}$.

We described a method that employs the thermal cycle test for choosing the appropriate geometry to be employed in rheological measurements of waxy crude oils. For our crude oil, we observed that the cone-and-plate geometry is not a good choice, because of the typically narrow gaps, which imply violation of the continuum hypothesis and affect the natural bulk crystallization process. The parallel-plate geometry with wide enough gaps was found to be more appropriate in our case. Thermal control was shown not to be a problem in our 
measurements, and we presented evidence that suggested that inhomogeneity is also unimportant. It is important to emphasize, however, that, in general, the best choice of geometry is expected to depend upon the specific oil at hand.

The starting temperature of the thermal cycle tests must also be chosen carefully, to ensure that crystallization occurs under the circumstances that are most critical to the industrial process of interest.

After the test conditions are defined with the aid of thermal cycle tests, it is possible to start a given rheological characterization, which should be preceded by the pretreatment, isothermal holding time, and cooling process.

The thermal cycle test results were shown to possess remarkable repeatability. Finally, we discussed several sources of errors, including changes in chemical composition, systematic errors, sample contraction, shear banding, apparent wall slip, and adhesive failure. 


\section{3}

\section{Post-cooling transient and steady-state measurements}

A fair complete rheological characterization of a Brazilian waxy crude oil is performed by using a recently proposed protocol to guarantee well defined shear and thermal histories to the samples. The effects of different histories on the post-cooling flow properties of the crude oil are investigated, considering both samples cooled under shear or statically. Four rheometric experiments, namely steady-state flow, constant shear rate, constant shear stress, and dynamic-stress-amplitude sweep are carried out and the results are presented in two chapters. This first chapter presents the results of constant shear stress, constant shear rate, and steady-state flow experiments. Interesting phenomena are observed, including non-monotonic flow curves, bifurcation of shear rate, dependence of the yield stress with thermal and shear histories, independence of the steady state results with the post-cooling initial condition of the experiment, and agreement between yield stress measured by different techniques. The contents of this chapter are going to be organized in one paper to be submitted to Journal of Rheology.

\section{1}

\section{Introduction}

Oil exploration in deepwater, ultra-deepwater, and arctic regions is expected to experience a substantial growth during next years because of world's increasing oil demand and scarcity of conventional oil reserves. This kind of oil exploitation brings a number of technology challenges, including the design of pipelines at low temperatures. Under such circumstances, the rheology of waxy crude oils changes dramatically, becoming extremely complex, and the main concern is wether or not the flow into a pipeline will restart after a long period of shutdown. Therefore, the accurate knowledge of the rheological properties of gelled waxy crude oils, in particular the yield stress, is of major importance to pipeline designers and operators.

Because of the increasing relevance of the subject to oil industry, a number of research works have been recently published $[36,55,69,70,71,72$, $73,74,75,76,77,78,79,80,81,82,83,84,85,86,87,88,89,90,91,92,93,94]$. 
Some of the main topics addressed by these papers are (i) the rheological characterization of gelled waxy oils, (ii) constitutive equations to describe the extremely complex behavior of these materials, and (iii) mathematical modeling of restart of gelled oil pipelines.

However, it can be noted in these papers that there is still no universally accepted protocol to perform the rheological characterization of waxy crude oils. In addition, there is no extensive enough rheological data obtained from one single oil submitted to different rheometric experiments and there is no constitutive equation available that can take into account all the reported behaviors for gelled waxy oils, including irreversible shear degradation and recovery of the structure with thermal cycling. Indeed, it is still possible to find in the literature disagreements with regard to the effects of holding time and thermal and shear histories on post-cooling oil rheology. Besides that, even definitions of the yield stresses and characteristic temperatures relevant to oil rheology can differ from paper to paper. Because of all the reasons listed above, it is clear that there is a lot of research remaining to be done in this field. There is a necessity to better understand the rheology of waxy crude oils at low temperatures to enable the development of new technologies required to increase production on these difficult-to-access areas.

In a previous publication [36] and Chapter 2 we described an experimental protocol designed to ensure well-defined thermal and shear histories of a waxy crude oil sample to be submitted to rheological measurements. Now we use the developed protocol to perform a fair complete rheological characterization of a Brazilian waxy crude oil. In this chapter, we describe the post-cooling transient and steady-state rheological measurements performed. Then, in Chapter 4 we show the post-cooling oscillatory measurements.

\section{2}

\section{Yielding and yield-stress measurements: an historical survey}

As reviewed by Barnes [95], yielding can be understood as an abrupt and profound change in the mechanical response of a material to a less resistant state under stress. This phenomenon is associated to a collapse of the microstructure and can be observed in a wide range of materials, including suspensions, emulsions, foams and polymer dispersions.

With respect to waxy crude oils, the understanding of the yielding phenomenon is fundamental for the successful design and operation of a pipeline. To restart the flow in a gelled oil pipeline, a pump pressure that gives a shear stress at the wall higher than the yield stress must be applied at the upstream of the pipeline. In addition, the shear stress at the wall must 
be high enough to give reasonable flow rates to clear the line in an acceptable time. Because of the importance of studying the yielding of gelled waxy oils, in this section we perform a review of the literature with regard to the yielding and yield-stress measurements of these materials.

In the literature, it can be noted that the study of the yielding of waxy oils has received attention since the 1950's, when an increase in oil gelling tendencies was observed and it was realized that the pour point cannot be used as a measure of pumpability of waxy oils [96, 53]. Thus, a more suitable physical property, called yield stress, have been chosen to define it and a systematic methodology for measuring this property of gelled waxy oils in a reliable way became necessary.

In the beginning of the 1970's, Davenport and Somper [35] carried out experiments using a full-scale, pilot-scale, and laboratory-model pipelines as well as a rotational viscometer with three different gaps concentric cylinders. They measured both the static $\left(\tau_{0 s}\right)$ and dynamic $\left(\tau_{0 d}\right)$ yield stresses of a Libyan crude oil and also obtained breakdown curves in different situations. The static yield stress was defined as the stress applied from rest in order to produce flow, or as later reported by Wardhaugh and Boger [46] "the stress at which fracture occurs", while the dynamic yield stress was taken as the extrapolated stress to the limiting zero shear rate in a flow curve - a shear stress-shear rate plot obtained from steady-state data.

Davenport and Somper [35] observed that the apparent viscosity obtained with rotational rheometry is higher than the one obtained with a pipeline apparatus, although the dynamic yield stress $\left(\tau_{0 d}\right)$ measured with both techniques presented good agreement. In addition, they [35] observed that neither changing the shear rate during cooling $\left(\dot{\gamma}_{h}\right)$ nor the gap width $(\delta)$ could cause any significant effect on the steady-state data ( $\downarrow$ or $\uparrow \delta$ or $\downarrow$ or $\uparrow \dot{\gamma}_{h} \Rightarrow \tau_{0 d}$ and $\eta_{S S} \approx$ constant), despite increasing the gap caused much better repeatability in the breakdown curves, which otherwise were gap dependent. With respect to the static yield stress $\left(\tau_{0 s}\right)$, Davenport and Somper [35] did not acquire repeatable results, but in general they found that the static yield stress increases as either the gap width or the pipe diameter $(D)$ decreases $\left(\downarrow \delta\right.$ or $\left.\downarrow D \Rightarrow \uparrow \tau_{0 s}\right)$. Based on the results obtained, Davenport and Somper [35] extended the breakdown theory of Billington [97] to explain the yielding of waxy crude oils by considering that the breakdown of the structure can be observed either in the viscosity or the static yield stress, which was assumed to decrease during yielding to a steady-state value.

Petrellis and Flumerfelt [44] also investigated the equilibrium and timedependent rheological properties of different waxy crude oils, by performing 
experiments under controlled thermal and shear histories in a Rheogoniometer, with cone and plate and Couette geometries. They reported that gelled waxy oils are shear degradable materials that present only a slight recovery of the structure after shear and a complete recovery after reheating and re-cooling. When dealing with the restart of gelled oil pipelines, Petrellis and Flumerfelt [44] emphasized the importance of obtaining yield-stress values from direct measurements $\left(\tau_{0 s}\right)$ rather than from extrapolation of the shear stress to a zero shear rate value in a flow curve $\left(\tau_{0 d}\right)$. They also pointed out that an accurate value of yield stress can be obtained only when the observation period is of the same order of that dictated by the expected application. This is one possible reason for the lack of repeatability in static yield stress measurements previously observed by Davenport and Somper [35].

In the beginning of the 1990's, Wardhaugh and Boger [46] performed a valuable discussion about the yielding of waxy crude oils and found out that the yielding of these materials is more complex than it had been previously considered in the literature. According to them, the yielding of waxy oils resembles the fracture of solids, which is quite different from the behavior of common viscoplastic and thixotropic materials. Particularly, unlike thixotropic materials [98], they observed that the microstructure of waxy crude oils at low temperatures cannot rebuild after a period of standing, provided that the temperature does not change [34, 46].

By fixing the cooling rate, Wardhaugh and Boger [46] performed constant shear rate, constant shear stress, oscillatory tests as well as used the vane technique in statically cooled samples of waxy crude oils and showed that the yielding process of these materials starts by a pure-elastic response, followed by a creep, which leads to a fracture-like behavior. During this yielding process, both an elastic yield stress $\left(\tau_{0 e}\right)$ and a static yield stress $\left(\tau_{0 s}\right)$ were identified. The elastic yield stress $\tau_{0 e}$ was defined as the stress at which the material behavior deviates from an elastic Hookean response and starts to creep.

Rønningsen [3] used mainly cone and plate rheometry to carry out constant shear stress, constant shear rate, and stress continuous ramp experiments to study the creep, yielding and time-dependent rheology of statically cooled samples of waxy crude oils. He [3] also investigated what he called "the time dependency" of the static yield stress by performing stress continuous ramps after applying either a certain stress or a fixed shear rate for increasing times. He [3] observed that the static yield stress decayed with a much larger rate constant after the application of a shear rate when compared to the tests performed after the application of a constant stress. In addition, Rønningsen [3] observed in both stress continuous ramp and constant shear rate experiments 
performed for different holding times after yielding $\left(t_{\text {holding_ay }}\right)$ that the structure partially rebuilds, but the original yield stress had hardly recovered even after $22 \mathrm{~h}$. By small temperature cycling, Rønningsen [3] also found that the structure partially rebuilds, but not as extensive as reported by Wardhaugh and Boger [33, 34].

For comparison with rotational rheometry, Rønningsen [3] studied the effects of cooling rate, holding time and stress loading rate on static yield stress in a model pipeline. By increasing the stress loading rate he measured higher yield stress values $\left(\uparrow \dot{\tau} \Rightarrow \uparrow \tau_{0 s}\right)$, the same trend observed with the rotational rheometry technique.

In the end of the 1990's, Chang et al. [52] have also studied the yielding of statically cooled samples of waxy crude oils with a fixed cooling rate. They emphasized the existence of a dynamic yield stress $\left(\tau_{0 d}\right)$, previously discussed by Davenport and Somper [35], Petrellis and Flumerfelt [44], and also by Rønningsen [3]. Unlike both the elastic and static yield stresses, which depend on the strength of the interlocking network of wax crystals before yielding, they argued that the dynamic yield stress is not a material property related to the yielding process itself, but is a parameter associated to the concentration and size of wax crystals that can be used to describe the oil properties at the final sheared state. Thus, they represented the yielding of waxy crude oils with the three-yield-stress concept presented by Kraynik [99] based on Houwink [100], and afterwards discussed by Bonnecaze and Brady [101] to explain the yielding behavior of electrorheological fluids.

Following the Rønningsen's work [3] and discussion, Chang et al. [52] have also investigated the "time dependency" of the three yield stresses. They found that the elastic limit yield stress is independent of the time scale and can be measured in creep-recovery and stress-amplitude-sweep tests, while both the dynamic and static yield stresses depend on it. The dynamic yield stress was measured in stress continuous ramps and was observed to decrease with increasing time (decreasing the stress loading rate $-\downarrow \dot{\tau} \Rightarrow \downarrow \tau_{0 d}$ ). Otherwise, the static yield stress was evaluated in all three kind of tests performed by Chang et al. [52], namely stress continuous ramps, creep-recovery and stressamplitude-sweep tests. In both stress continuous ramps and creep-recovery tests the static yield stress followed the same trend observed for the dynamic yield stress and also found by Rønningsen [3], i.e. decrease with increasing time (which means decreasing the stress loading rate and increasing the creep time respectively $-\downarrow \dot{\tau}$ or $\left.\uparrow t_{\text {creep }} \Rightarrow \downarrow \tau_{0 d}\right)$. As a difference from Rønningsen's results [3] that show that the static yield stress probably vanish in the limit of very low stress loading rates, Chang et al. [52] found that the elastic yield 
stress is the inferior limit to the static one. On the contrary, unlike this last trend observed in both stress continuous ramps and creep-recovery tests, in stress-amplitude-sweep tests Chang et al. [52] observed that the static yield stress decreased with increasing the frequency $\left(\uparrow f \Rightarrow \downarrow \tau_{0 s}\right)$, which corresponds to a decrease in the time scale. This last behavior was explained by the fact that more vibration in the test causes more damage to the waxy structure, leading to a lower yield stress value.

From stress-amplitude-sweep tests, Webber [58] found similar yielding behavior to that observed by Wardhaugh and Boger [46] and also by Chang et al. [52] for statically cooled samples of waxy crude oils, consisting of an elastic-solid response, creep and then fracture. He [58] showed that imposing a stress/strain history to a sample of waxy oil can cause a qualitatively change in the storage and loss moduli response. A small oscillatory strain history cause a reduction in $G^{\prime}$ when compared to statically cooled samples, but neither affect the elastic-solid response characteristic of waxy oils followed by a fracture, nor the static yield stress and the corresponding yield strain, which remain nearly constant. However, a large-oscillatory-strain history or a continuous-shear-rate history strongly affect the macroscopic structure and change this behavior, causing a strong decrease on both $G^{\prime}$ and $G^{\prime \prime}$ and turning $G^{\prime \prime}$ higher than $G^{\prime}$, without the typical yielding, despite keeping the stress-history-dependent behavior.

All the above discussed works show how complex is the yielding of gelled waxy oils compared to a regular viscoplastic material. Three different yield stresses related to gelled waxy oils were reported, namely elastic, static, and dynamic yield stresses. It was assumed in some of the works discussed above, a time-dependency of the static and dynamic yield stresses. However, if the dynamic yield stress is evaluated from extrapolation of steady-state data it is clear that this property will not depend on time. Therefore, it is important to point out that (i) we need to separate what is the material property from what is the influence of the technique used to evaluate the material property, (ii) we need to be aware about the definitions used by different authors, and (iii) we need to be careful when using these yield-stress values for pipeline design. In addition, there is still a need of investigating the effects of thermal and shear histories and holding time on the yield stresses obtained. So, a greater amount of measurements with different kinds of waxy oils in a variety of conditions are required to better understand the yielding of these materials. Only after that, it will be possible to propose a robust mathematical model that can accurately predict the yielding and all of these trends reported for gelled waxy oils. 


\section{3 \\ Materials and methods}

A Brazilian waxy crude oil provided by PETROBRAS was used in the research described in this chapter. Samples of this crude oil were prepared for the post-cooling rheological measurements according to a recently proposed protocol to guarantee well defined thermal and shear histories [36].

The sample preparation procedure consists on three main steps, (i) the pretreatment that ensures a stable chemical composition, (ii) the isothermal holding time to dissolve all wax crystals and erase the thermal memory of the oil, and (iii) the cooling process that must be carried out with appropriate geometry, gap, and temperature range for the given waxy oil and application.

The pretreatment applied to the Brazilian waxy crude oil was heating the oil in an opened bottle for $3 \mathrm{~h}$ at $50^{\circ} \mathrm{C}$ and then for $1 \mathrm{~h}$ at $60^{\circ} \mathrm{C}$ to evaporate light ends. After that, the pretreated oil was divided, while at $60^{\circ} \mathrm{C}$, in twelve closed bottles and stored at ambient temperature. Before the beginning of each post-cooling rheometric test, a sample was collected from one bottle, after homogenizing the oil, and loaded into a parallel plates geometry. Then, an isothermal holding time of $30 \mathrm{~min}$ at $50^{\circ} \mathrm{C}$ was applied to the sample to dissolve the wax crystals. After that, the cooling process was carried out to guarantee that the waxy crude oil samples achieve the measurement temperature under controlled shear and thermal histories. Two rheometers from TA Instruments equipped with peltier systems were used in the investigation presented in this chapter, an AR-G2 with a $60 \mathrm{~mm}$ parallel plates and an ARES-G2 with a $50 \mathrm{~mm}$ parallel plates. More detailed information about the criteria of choosing the pretreatment, geometry, gap, and starting temperature can be found in Chapter 2 or elsewhere [36].

\section{4}

\section{Results and discussion}

In this section we present the results of the post-cooling constant shear stress, constant shear rate, and steady-state flow rheometric experiments performed with the Brazilian waxy crude oil investigated. All the tests start immediately after cooling the sample to the measurement temperature $T_{0}$, unless specified otherwise. The cooling process is performed according to the previously discussed protocol (Chapter 2) to ensure well-defined shear and thermal histories to the samples, expressed by the shear rate during cooling, $\dot{\gamma}_{h}$, and the cooling rate, $\dot{T}_{h}$, respectively. 


\subsection{1 \\ Constant shear stress rheometric experiments}

The constant shear stress rheometric experiments consist on imposing a constant stress to a gelled waxy oil sample and evaluating how the strain and/or the shear rate evolves with time. The results of several experiments performed by imposing different constant stresses on both samples cooled statically or under shear are plotted in Figs. 3.1, 3.2, 3.3, and 3.4 and discussed below.

\section{Statically cooled samples}

The results of the constant stress tests performed with samples statically cooled at $1^{\circ} \mathrm{C} / \mathrm{min}$ are plotted in Fig. 3.1, in which the strain-versus-time plot can be found in Fig. 3.1(a) and the shear-rate-versus-time plot can be found in Fig. 3.1(b).

On one hand, an analysis of the results show that no flow is observed by imposing 1 or $2 \mathrm{~Pa}$ to the samples, because after an initial transient, the strain response from both tests stop changing with time in Fig. 3.1(a), which means that the corresponding shear rates goes to zero in Fig. 3.1(b). Thus, when a constant stress up to $2 \mathrm{~Pa}$ is applied to a sample, statically cooled at $1^{\circ} \mathrm{C} / \mathrm{min}$, only elastic deformation is observed, which means that the material behaves as a Hookean elastic solid in this stress range.

In fact, it can be argued that if long enough times are waited, perhaps flow can be observed even for applied stresses below $2 \mathrm{~Pa}$. However, it is not possible to observe any changes in strain for applied stresses below $2 \mathrm{~Pa}$, even after $100,000 \mathrm{~s} \approx 27 \mathrm{~h}$ in Fig. 3.1(a). Therefore, it is reasonable to assume that no flow will be observed in this range of applied stresses during the time scale of a production shutdown in a pipeline, the present application.

On the other hand, a different behavior is observed for applied stresses higher than $4 \mathrm{~Pa}$ in the same Fig. 3.1. In this case, for a constant applied stress, the strain keeps increasing with time after an initial transient, which means that plastic deformation and flow is observed. Moreover, it can be seen that the strain curves achieve approximately constant inclinations after a given time in Fig. 3.1(a), which is an evidence that the steady state is achieved. The corresponding shear-rate results for applied stresses higher than $4 \mathrm{~Pa}$ are the approximately constant shear-rate curves obtained after an initial transient in Fig. 3.1(b), which corroborates the hypothesis that the steady state has been achieved.

Thus, in summary, for samples cooled under $\dot{\gamma}_{h}=0$ and $\dot{T}_{h}=1{ }^{\circ} \mathrm{C} / \mathrm{min}$, no flow is observed if stresses lower than or equal to $2 \mathrm{~Pa}$ are applied. However, flow 


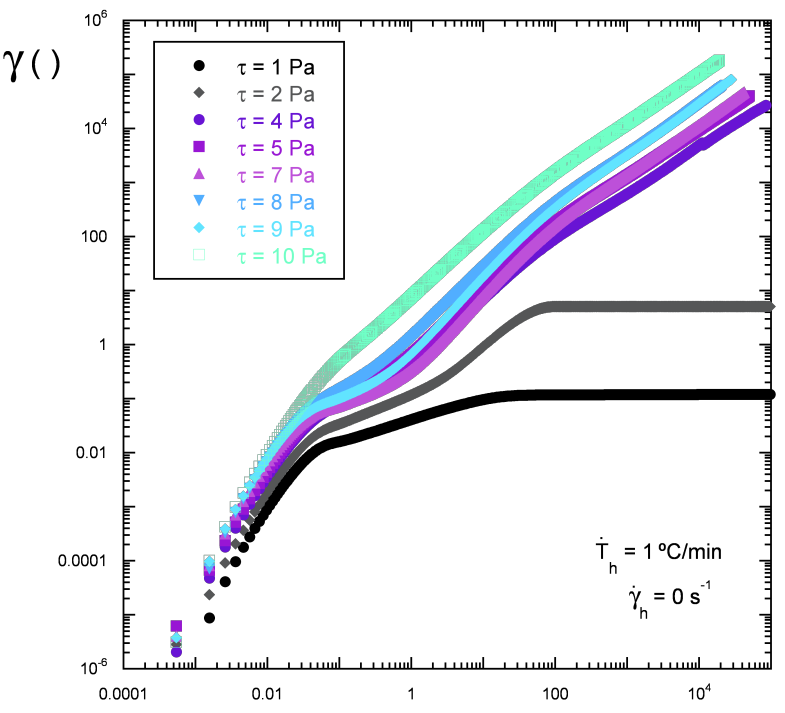

(a)

Time (s)

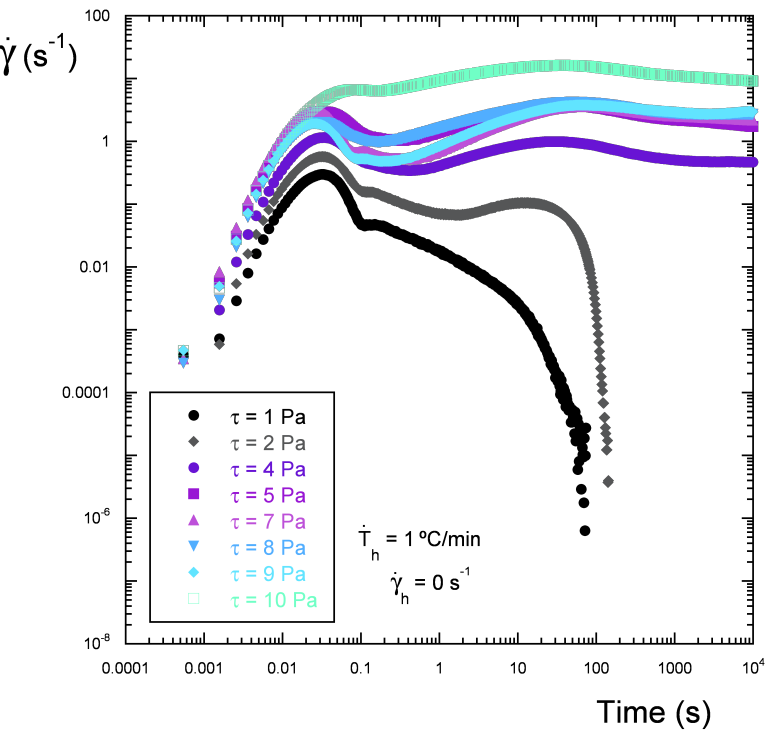

(b)

Figure 3.1: Post-cooling constant shear stress tests performed on statically cooled samples, $\dot{\gamma}_{h}=0 \mathrm{~s}^{-1}$ and $\dot{T}_{h}=1^{\circ} \mathrm{C} / \mathrm{min}$ : (a) strain-versus-time plot and (b) shear-rate-versus-time plot.

is observed if stresses higher than or equal to $4 \mathrm{~Pa}$ are applied. The two different behaviors for the different ranges of applied stresses cause a bifurcation in both strain and shear-rate plots when several experiments are carried out, as observed in Figs. 3.1(a) and 3.1(b). With this technique we can estimate the yield stress of the gelled oil for the given $\dot{\gamma}_{h}$ and $\dot{T}_{h}$ between 2 and $4 \mathrm{~Pa}$, or roughly $3 \mathrm{~Pa}$.

It is important to note that two different yield stresses were obtained by Chang et al. [52] from creep-recovery experiments performed with statically cooled samples, namely, elastic and static yield stresses. They argued that for a given observation time, depending on the stress applied, three different be- 
haviors can be observed, (i) the material can experience an elastic deformation followed by a complete recovery when the stress is removed, (ii) the material can experience an elasto-plastic deformation followed by only partial recovery when the stress is removed, and (iii) the material can yield or fracture.

They defined the two yield stresses based on the three possible behaviors, an elastic yield stress that is the limiting stress between the behaviors (i) and (ii), and a static yield stress that is the limiting stress between the behaviors (ii) and (iii). Moreover, they pointed out that the elastic yield stress is the inferior limit to the static yield stress for long enough observation times and that while the elastic yield stress is independent on time, the static yield stress is a time-dependent property.

Nevertheless, the classical definition of yield stress is the limiting stress above which the material behaves as a liquid and flow is observed and below which the material behaves as a solid and no flow is observed. In this way, it becomes clear that the elastic yield stress, as defined, respect the classical definition of yield stress, being a true material property. However, the static yield stress, as defined in the literature of waxy crude oils, can be understood as a rough measurement of the yield stress, because this measurement is a function of the rheometric experiment performed and cannot be considered a true material property. In this way, as we assume that the experiments are performed with long enough observation times for the present application, the above estimate for the yield stress based on bifurcation results, corresponds, in fact, to the elastic yield stress.

\section{Samples cooled under shear}

With respect to the samples cooled at $\dot{\gamma}_{h}=20 \mathrm{~s}^{-1}$ and $\dot{T}_{h}=1^{\circ} \mathrm{C} / \mathrm{min}$, constant stress tests were performed (i) to investigate the effects of the initial condition on the results, (ii) to evaluate the elastic yield stress, and (iii) to investigate the response obtained by imposing exactly the estimated elastic yield stress to different samples submitted to the same history.

The initial condition of a post-cooling rheometric test that starts immediately after reaching the measurement temperature $T_{0}$ is characterized by $\dot{\gamma}_{h}$, because in this case at the time $t=0 \mathrm{~s}$ the gelled waxy oil sample is submitted to a shear rate equal to the shear rate applied during cooling. Thus, it is expected that if a constant stress test is carried out immediately after reaching $T_{0}$, the shear rate will change from $\dot{\gamma}_{h}$ at $t=0 \mathrm{~s}$ to another value that depends on the stress imposed. This initial condition is different from the one of statically cooled samples, in which the shear rate increases from $0 \mathrm{~s}^{-1}$ to another value that also depends on the stress imposed. As we intend to compare only 
the effects of shear history on the results and the difference in the initial condition of the test leads to a different initial transient response, it is important to first investigate how the initial condition can affect the results.

To this end, we compare tests with samples submitted to the same history, performed immediately after reaching $T_{0}$, to tests performed $1 \mathrm{~s}$ after reaching $T_{0}$, as illustrated in Fig. 3.2. During this $1 \mathrm{~s}$ after cooling to $T_{0}$, the sample is kept at rest, so that, in this case, the initial condition of the constant stress test is a shear rate equal to $0 \mathrm{~s}^{-1}$, even though $\dot{\gamma}_{h}=20 \mathrm{~s}^{-1}$. This $1 \mathrm{~s}$ is enough to change the initial condition, but it is reasonable to assume that no significant changes in the structure of the gelled oil occurs during this short period of time, thus allowing for the investigation of only the effects of the initial condition on the results.

It can be seen in Figs. 3.2(a) and 3.2(b) that for three different applied stresses, the initial transient changes with the initial condition, as expected, but after a while no significant differences can be seen when comparing the two strain- or shear-rate-curves pertaining to each applied stress. Therefore, it is possible to conclude that, at least in the ranges investigated, the differences in initial condition does not affect the final result of each test, which implies that the yield stress measurement is also not affected by the initial condition of the tests.

Thus, to allow for a better comparison, we decided to evaluate the elastic yield stress of both samples cooled statically or under shear, by performing constant stress tests with the initial condition equal to $0 \mathrm{~s}^{-1}$. The bifurcation results of strain and shear rate, pertaining to samples cooled at $\dot{\gamma}_{h}=20 \mathrm{~s}^{-1}$ and $\dot{T}_{h}=1{ }^{\circ} \mathrm{C} / \mathrm{min}$, can be found in Figs. 3.3(a) and 3.3(b), respectively.

From this figures it can be observed that after an initial transient, for applied stresses lower or equal to $0.8 \mathrm{~Pa}$, the strain keeps constant and the shear rate goes to zero, which means that no flow is observed. Nevertheless, for applied stresses higher or equal to $1 \mathrm{~Pa}$, the strain achieves an approximately constant inclination and the shear rate goes to a finite value. This means that, not only flow is observed, but also a steady state is achieved for the applied stress. Thus, for samples cooled at $\dot{\gamma}_{h}=20 \mathrm{~s}^{-1}$ and $\dot{T}_{h}=1{ }^{\circ} \mathrm{C} / \mathrm{min}$, the estimated yield stress value of the gelled crude oil is $0.9 \mathrm{~Pa}$.

After estimating the yield stress value for this case, it is interesting to observe what happen to different samples, submitted to the same history, when a constant stress equal to the yield stress is applied. The results of six tests performed with different samples are plotted in Figs. 3.4(a) and 3.4(b). It is curious to note in these figures that, in three tests, it was observed flow and in other three tests no flow was observed, reflecting an unstable situation in 


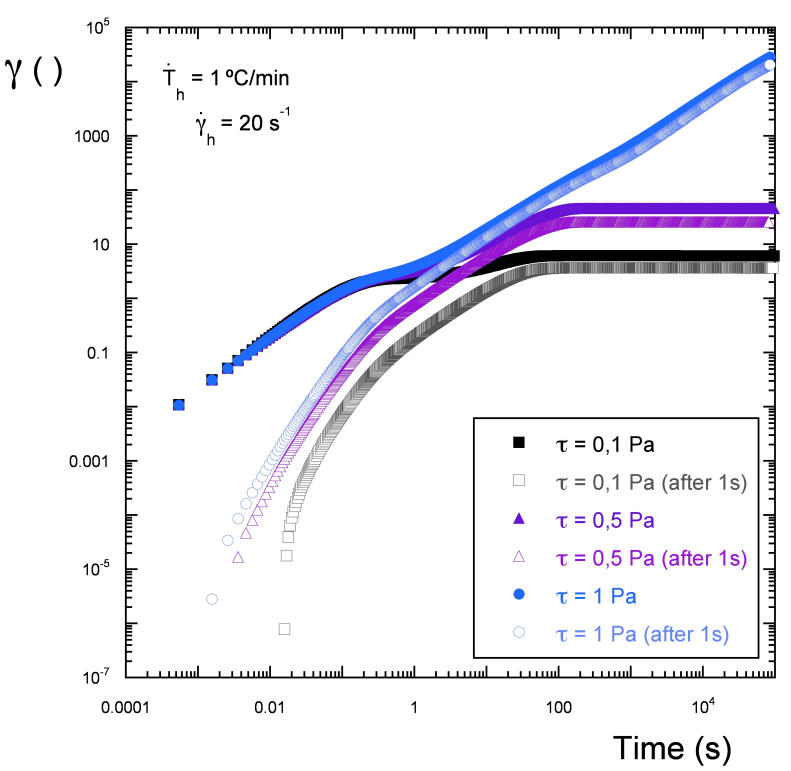

(a)

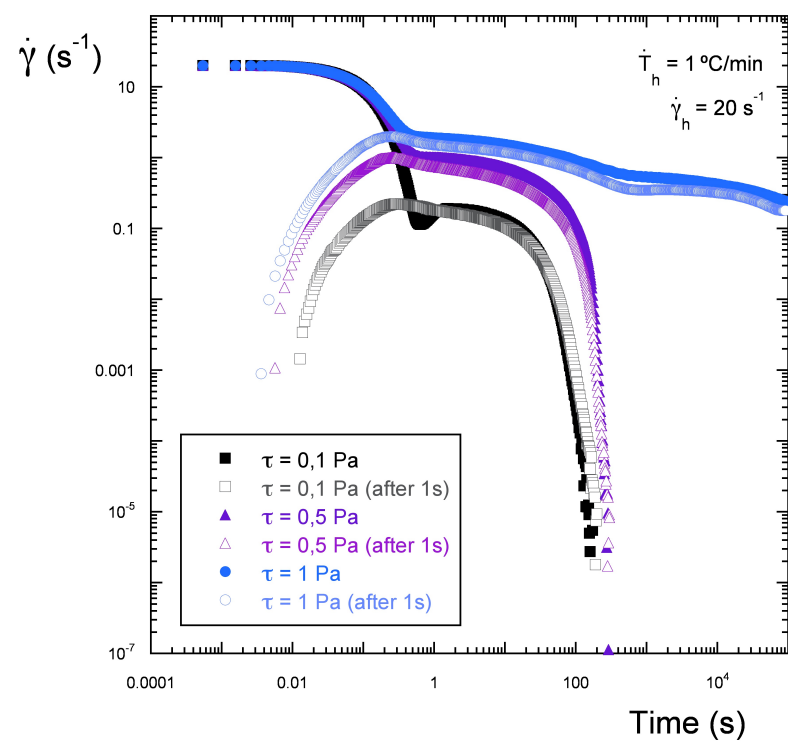

Figure 3.2: Post-cooling constant shear stress tests performed on samples cooled under shear, $\dot{\gamma}_{h}=20 \mathrm{~s}^{-1}$ and $\dot{T}_{h}=1{ }^{\circ} \mathrm{C} / \mathrm{min}$ : investigation of the effects of changing the initial condition on the results, (a) strain-versus-time plot and (b) shear-rate-versus-time plot. 


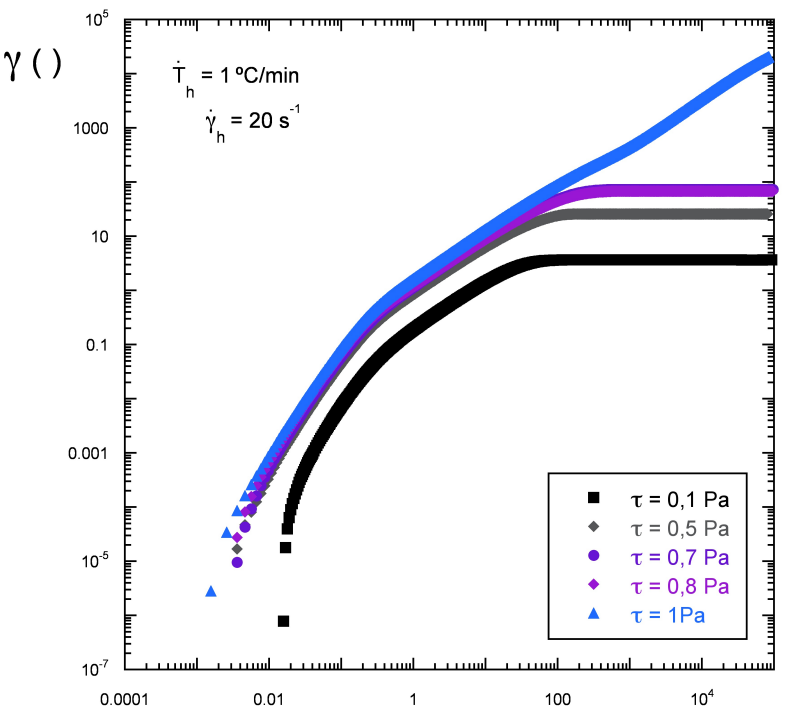

(a)

Time (s)

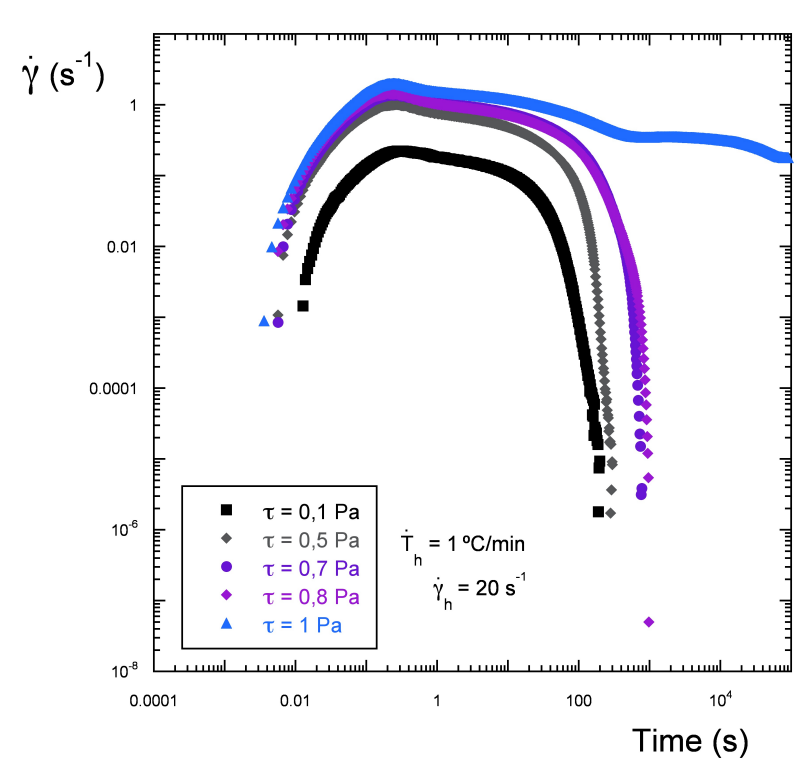

Figure 3.3: Post-cooling constant shear stress tests performed on samples cooled under shear, $\dot{\gamma}_{h}=20 \mathrm{~s}^{-1}$ and $\dot{T}_{h}=1{ }^{\circ} \mathrm{C} / \mathrm{min}$ : (a) strain-versus-time plot and (b) shear-rate-versus-time plot.

which every small instability can cause yielding and flow. This is an evidence that, indeed, the yield stress is approximately $0.9 \mathrm{~Pa}$ in this case. 


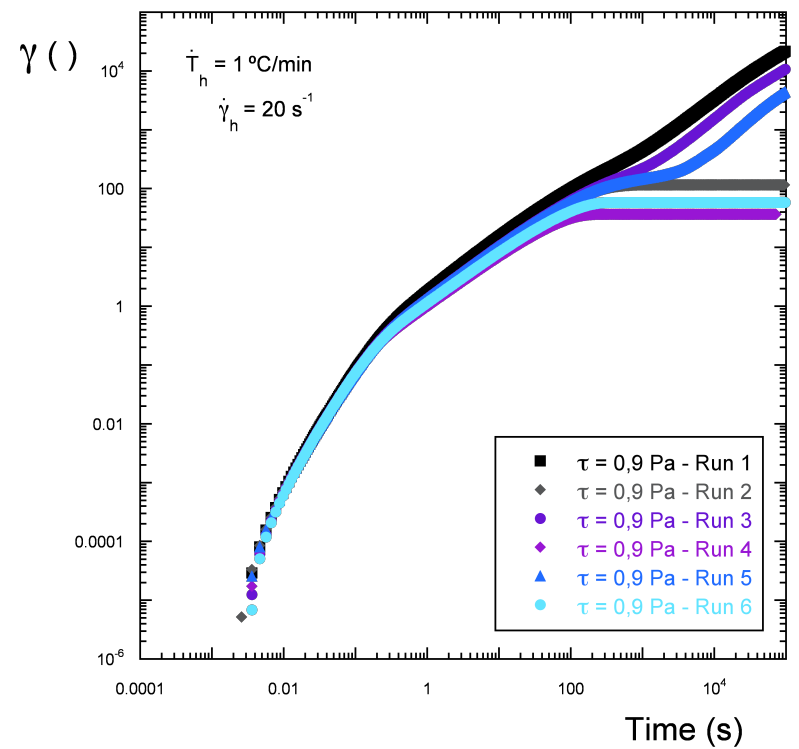

(a)

Time (s)

(b)

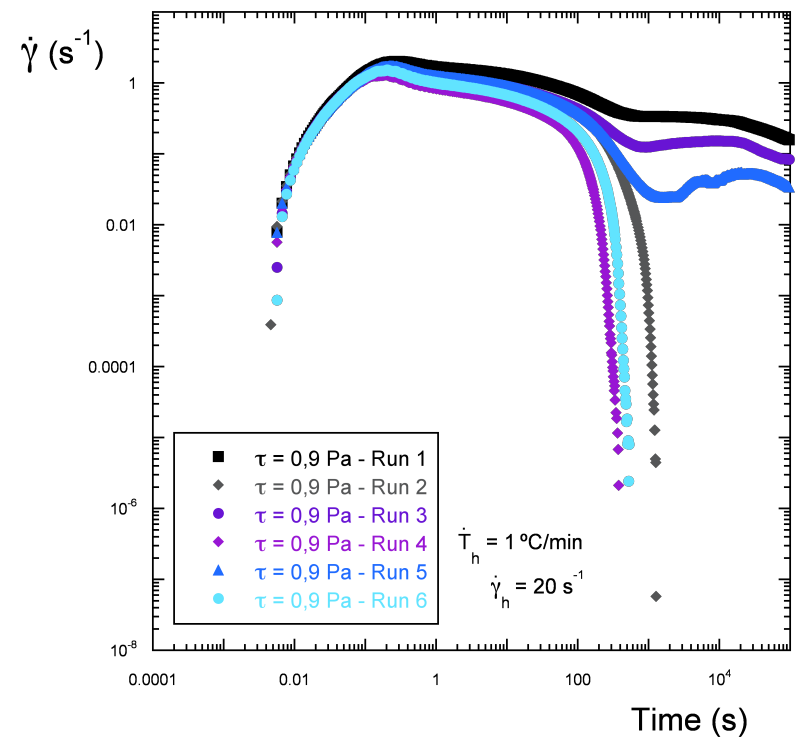

Figure 3.4: Post-cooling constant shear stress at $0.9 \mathrm{~Pa}$ applied on samples cooled under shear, $\dot{\gamma}_{h}=20 \mathrm{~s}^{-1}$ and $\dot{T}_{h}=1{ }^{\circ} \mathrm{C} / \mathrm{min}$ : (a) strain-versus-time plot and (b) shear-rate-versus-time plot.

\section{4 .2}

\section{Constant shear rate rheometric experiments}

The constant shear rate rheometric experiments consist on imposing a constant shear rate to a gelled waxy oil sample and evaluating how the stress and/or the viscosity evolves with time. The results of several experiments performed by imposing different constant shear rates to samples cooled under shear are plotted in Fig. 3.5.

From this figure, it can be observed that for applied shear rates equal or higher than $2 \mathrm{~s}^{-1}$, the stress, and consequently the viscosity, changes only slightly with time, until a steady state is achieved. In particular, the curves 
pertaining to applied shear rates of $20 \mathrm{~s}^{-1}$ and $200 \mathrm{~s}^{-1}$ show a slight decrease of stress with time, while the curve pertaining to $2 \mathrm{~s}^{-1}$ show a slight increase of stress with time. This behavior is probably due to the fact that the constant shear rate tests start immediately after cooling the sample to the measurement temperature at $\dot{\gamma}_{h}=20 \mathrm{~s}^{-1}$. Thus, at the beginning of the test, the shear rate changes abruptly from $20 \mathrm{~s}^{-1}$, the shear rate during cooling, to the applied constant rate after cooling. In the case that the applied shear rate is $200 \mathrm{~s}^{-1}$, additional break down of the microstructure occurs because of the increase in shear rate, which causes a slight decrease in stress with time. Nevertheless, when the applied shear rate is $2 \mathrm{~s}^{-1}$, the structure can experience a buildup because of the decrease in shear rate, which can explain the slight increase of stress with time in this case.

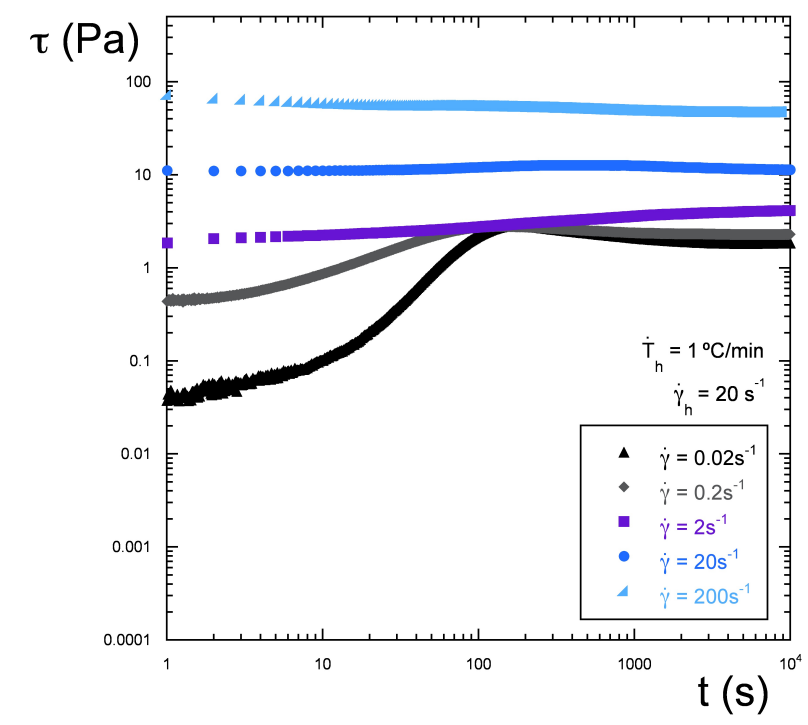

Figure 3.5: Post-cooling constant shear rate tests performed on samples cooled at $\dot{\gamma}_{h}=20 \mathrm{~s}^{-1}$ and $\dot{T}_{h}=1{ }^{\circ} \mathrm{C} / \mathrm{min}$.

In the same figure, the curves pertaining to shear rates equal to $0.2 \mathrm{~s}^{-1}$ and $0.02 \mathrm{~s}^{-1}$ show a different behavior. In both cases the stress increases with time until a maximum level is achieved, reflecting a build up of the structure, but after this point the stress exponentially decreases with time until the steady state is achieved. Two factors can contribute to this observed behavior, (i) the previously mentioned abrupt change in shear rate that can give rise to a build up of the structure and (ii) the elasticity and thixotropy of the material.

Irrespectively to the applied shear rate after cooling, the shear stress becomes approximately constant after a certain time in all cases, indicating that the steady state is achieved. If we plot this steady state stress for each shear rate applied, we can build the flow curve of the gelled oil cooled at $\dot{\gamma}_{h}=$ 
$20 \mathrm{~s}^{-1}$ and $\dot{T}_{h}=1{ }^{\circ} \mathrm{C} / \mathrm{min}$, as previously discussed by Wardhaugh and Boger $[33,34]$ and shown in the next section.

\subsection{3}

\section{Steady-state flow rheometric experiments}

The steady-state flow rheometric experiments consist on building the flow curve of a given material. In this section two possible ways of obtaining a flow curve are presented and compared. The first way is to build a flow curve with the steady-state points obtained from constant shear rate experiments as discussed in the last section and the second way is to carry out a step rate sweep test, in which the shear rate changes in steps and the stress is recorded for each shear rate applied after achieving a steady-state criteria.

The two possible ways of obtaining the flow curve of the gelled oil cooled under shear at $\dot{\gamma}_{h}=20 \mathrm{~s}^{-1}$ and $\dot{T}_{h}=1^{\circ} \mathrm{C} / \mathrm{min}$ are presented in Fig. 3.6. It is possible to note in this figure a reasonably good agreement between the curves obtained with the two methods. It can also be observed that the gelled waxy oil cooled under this shear and thermal histories present a flow curve of a viscoplastic material with an extrapolated dynamic yield stress slightly higher than $1 \mathrm{~Pa}$.

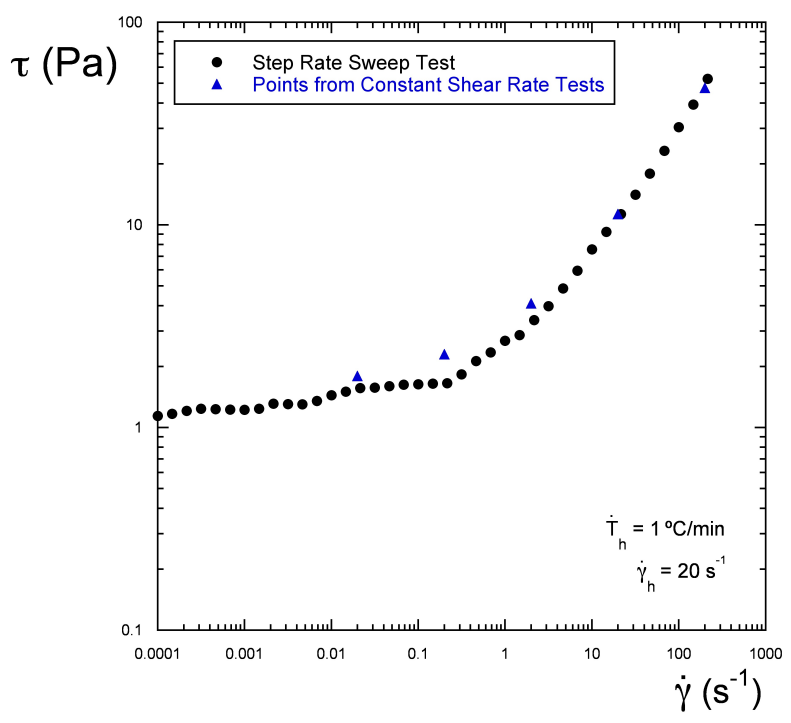

Figure 3.6: Post-cooling flow curve of samples cooled at $\dot{\gamma}_{h}=20 \mathrm{~s}^{-1}$ and $\dot{T}_{h}=$ $1{ }^{\circ} \mathrm{C} / \mathrm{min}$.

From the constant stress experiments presented in Sec. 3.4.1 we could infer that the elastic yield stress of the gelled waxy oil cooled at $\dot{\gamma}_{h}=20 \mathrm{~s}^{-1}$ and $\dot{T}_{h}=1^{\circ} \mathrm{C} / \mathrm{min}$ is $0.9 \mathrm{~Pa}$. This value is in reasonably good agreement with the extrapolated dynamic yield stress obtained. The small difference in the yield stresses obtained from the two different techniques can be attributed to the 
imprecision inherent to the extrapolation technique as well as to experimental uncertainties. Therefore, it is reasonably to assume that the Brazilian waxy crude oil cooled under $20 \mathrm{~s}^{-1}$ and $1{ }^{\circ} \mathrm{C} / \mathrm{min}$ present one single yield stress around $1 \mathrm{~Pa}$ and a behavior of a regular yield-stress material. This behavior is in conformity with a microstructure formed by a regular suspension of wax crystals.

It is important to note that the elastic yield stress obtained from constant stress experiments performed with statically cooled samples is roughly $3 \mathrm{~Pa}$, three times higher than the yield stress of the gelled oil cooled at $20 \mathrm{~s}^{-1}$ and $1{ }^{\circ} \mathrm{C} / \mathrm{min}$. Also, preliminary experiments, not shown in this thesis, reveal that the dynamic yield stress of statically cooled samples, extrapolated from steadystate data, is roughly equal to the one obtained from samples cooled under shear, $\approx 1 \mathrm{~Pa}$. This observation is supported by previous conclusions found in the literature that steady-state flow properties are not very sensitive to the thermal and shear histories experienced by the sample [33, 34, 35, 31] in the range of high enough shear rates. Thus, it seems that statically cooled samples of the Brazilian waxy crude oil investigated present a non-monotonic flow curve with two yield stresses, roughly equal to 1 and $3 \mathrm{~Pa}$. Unfortunately, at this time we cannot provide evidence to support this hypothesis.

\section{5 \\ Final remarks}

In this chapter we presented the results of post-cooling constant shear stress, constant shear rate, and steady-state flow experiments performed with the Brazilian waxy crude oil studied. We investigated the history effects on the rheology of this material and evaluated the yield stress of samples cooled under shear or statically.

From constant stress experiments we observed that changes in the initial condition of the test do not affect the final result of the experiment. In addition, we observed a bifurcation on strain and shear rate plots and estimated the yield stress of statically cooled samples as $3 \mathrm{~Pa}$ and the yield stress of samples cooled at $20 \mathrm{~s}^{-1}$ as $0.9 \mathrm{~Pa}$.

The constant shear rate tests helped us in obtaining the time required for each applied shear rate to achieve the steady state. With these steadystate values we built a flow curve of samples cooled under shear and compared this result with the flow curve obtained from step rate sweep experiments. Both curves are in reasonably good agreement, showing a behavior of a typical viscoplastic material. In addition, we could estimate the yield stress of samples cooled under shear based on extrapolation of stress in the flow curve to the 
limit of zero shear rate. In this case, the estimated yield stress is slightly above $1 \mathrm{~Pa}$, which is not very different from the $0.9 \mathrm{~Pa}$ previously obtained.

At last, we could observe that for high enough shear rates the steady state result does not depend significantly on the previous history experienced. Thus, the extrapolated yield stress value for statically cooled samples is expected to be similar to the one obtained for samples cooled under shear, slightly above $1 \mathrm{~Pa}$. As the yield stress of statically cooled samples obtained from bifurcation results is roughly $3 \mathrm{~Pa}$, a non-monotonic flow curve is expected for statically cooled samples. Nevertheless, additional experiments must be performed to verify this possibility. 


\section{Post-cooling oscillatory measurements}

In this chapter we present the post-cooling stress amplitude sweep tests performed with the Brazilian waxy crude oil by using a recently proposed protocol to guarantee well defined shear and thermal histories to the samples. As pointed out in the previous chapter, samples cooled under shear or statically are examined. The yielding as well as the effects of holding time, frequency, and thermal and shear histories on rheology of the gelled oil are investigated in the light of this experimental technique. The contents of this chapter are going to be organized in a second paper to be submitted to Journal of Rheology.

\section{1 \\ Introduction}

As previously discussed, at low enough temperatures, waxy crude oils present a complex rheological behavior, caused by the formation of an interlocking network of wax crystals, that is dependent on the shear and thermal histories experienced by the gelled oil. In Chapter 3, we highlighted the importance of obtaining accurate measurements of the rheological properties of gelled waxy oils to pipeline design. In addition, we presented post-cooling steady-state flow, constant shear rate, and constant shear stress rheometric experiments. With those experiments we could obtain yield stress values for the Brazilian gelled waxy crude oil and have insight into its complex rheological behavior, that includes history and time dependency, elasticity, and yielding.

In this chapter, we present results of post-cooling oscillatory stress amplitude sweep experiments, performed to evaluate the yield stress(es), to investigate the yielding process, and to better understand different effects on wax structure and rheology, such as, history, frequency, and holding time. The main purposes of this stage of investigation are (i) to obtain extensive rheological data of the Brazilian gelled waxy oil that can be used in future developments of constitutive equations and (ii) to compare the yield stress(es) obtained from oscillatory measurements to the yield stress(es) obtained from the other rheometric experiments shown in Chapter 3. This comparison can enable us to choose one technique that provides accurate yield-stress 
measurements without being too much time consuming. This is desirable and useful to pipeline design.

\section{2 \\ Materials and methods}

The rheometric experiments described in this chapter were performed with the aid of an AR-G2 rheometer from TA Instruments equipped with smooth parallel plates geometry, being the upper-plate diameter equal to $60 \mathrm{~mm}$ and the gap varying between 2 and $3 \mathrm{~mm}$ depending on $\dot{\gamma}_{h}$ and $\dot{T}_{h}$. No special profiled or roughened device was employed because apparent wall slip was found to be negligible during measurements with the material tested [36]. The pretreatment consisted on heating the crude oil at $50^{\circ} \mathrm{C}$ for $3 \mathrm{~h}$ and then at $60^{\circ} \mathrm{C}$ for $1 \mathrm{~h}$ in an opened bottle. The pretreated oil was stored at ambient temperature $\left(\approx 23^{\circ} \mathrm{C}\right)$ in twelve closed bottles and each sample was collected from one bottle after carefully mixing it to homogenize possible suspended wax crystals. More detailed information about the pretreatment, choice of gap, geometry, starting temperature, and experimental protocol can be found in Chapter 2 or elsewhere [36]. Each post-cooling stress amplitude sweep test presented in this chapter was performed after cooling the sample from the starting temperature $T_{i}=50^{\circ} \mathrm{C}$, to the measurement temperature $T_{0}=4^{\circ} \mathrm{C}$, under strict control of the cooling rate $\dot{T}_{h}$ and shear history, expressed by $\dot{\gamma}_{h}$. All the stress amplitude sweep tests were performed with a constant frequency equal to $1 \mathrm{~Hz}$, unless specified otherwise.

\section{3 \\ Results and discussion}

\subsection{1 \\ Description of the post-cooling stress amplitude sweep test and its main parameters}

The stress amplitude sweep test starts after reaching the measurement temperature $T_{0}$. The test is an oscillatory rheometric experiment performed under a fixed frequency, in which the stress amplitude increases from small to large values. Two possible plots from this kind of experiment can be seen in Figs. 4.1(a) and 4.1(b). Figure 4.1(a) illustrates the storage and loss moduli as a function of stress amplitude, while Fig. 4.1(b) shows the stress amplitude as a function of strain, as earlier shown by Chang et al. and Webber [52, 37, 58].

In Fig. 4.1(a) it can be observed a linear elastic storage modulus $\left(G_{e}^{\prime}\right)$ and a linear elastic loss modulus $\left(G_{e}^{\prime \prime}\right)$. In addition, the elastic yield stress $\left(\tau_{0 e}\right)$ is usually defined as the stress amplitude at which a deviation from the linear 
(a)
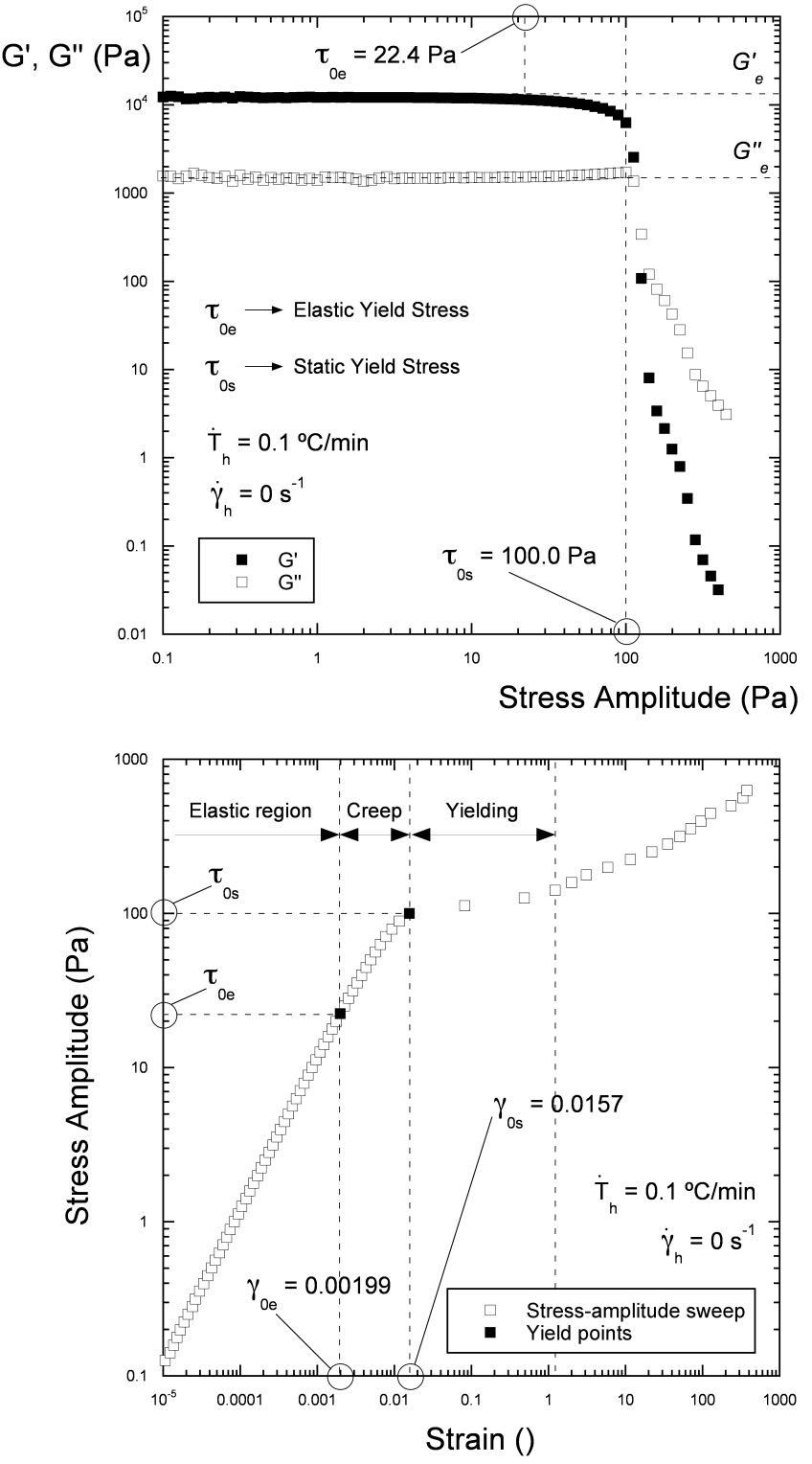

(b)

Figure 4.1: Stress-amplitude sweep test: (a) $G^{\prime}$ and $G^{\prime \prime}$ as a function of stress amplitude and (b) stress amplitude as a function of strain - main parameters.

elastic regime can be identified, in spite of existing some imprecision in this measurement. In our case, we considered that the elastic yield stress is obtained from a stress amplitude versus strain plot (Fig. 4.1(b)) with a deviation criteria of $5 \%$ with respect to the linear relation between them. Besides that, in Fig. 4.1(a), the static yield stress $\left(\tau_{0 s}\right)$ is defined as the point beyond which the storage modulus $\left(G^{\prime}\right)$ experience a dramatic drop, even though many authors consider it as the point in which $G^{\prime}$ becomes equal to $G^{\prime \prime}$. Both elastic and static yield points are also plotted in Fig. 4.1(b), where an elastic yield strain $\left(\gamma_{0 e}\right)$ and also a static yield strain $\left(\gamma_{0 s}\right)$ can be identified respectively $[31,58]$. These are the main parameters that can be measured in a stress-amplitude sweep test, in which three zones can be identified, namely, elastic region, creep, and 
the region in which the yielding phenomenon itself or fracture can be observed.

\subsection{2}

\section{Effects of shear and thermal histories on structure and rheology}

After describing the stress amplitude sweep test and identifying the elastic and static yield points, we can investigate the effects of shear and thermal histories on the results to better understand the material behavior. To this end, samples were prepared under different cooling conditions, according to a defined shear and thermal history. In all graphics, $G^{\prime}$ is represented by the filled markers and $G^{\prime \prime}$ by the empty markers. In this set of results, plotted in Figs. 4.2, 4.3, 4.4, and 4.5, the post-cooling stress amplitude sweep tests were performed immediately after reaching the measurement temperature $T_{0}$.

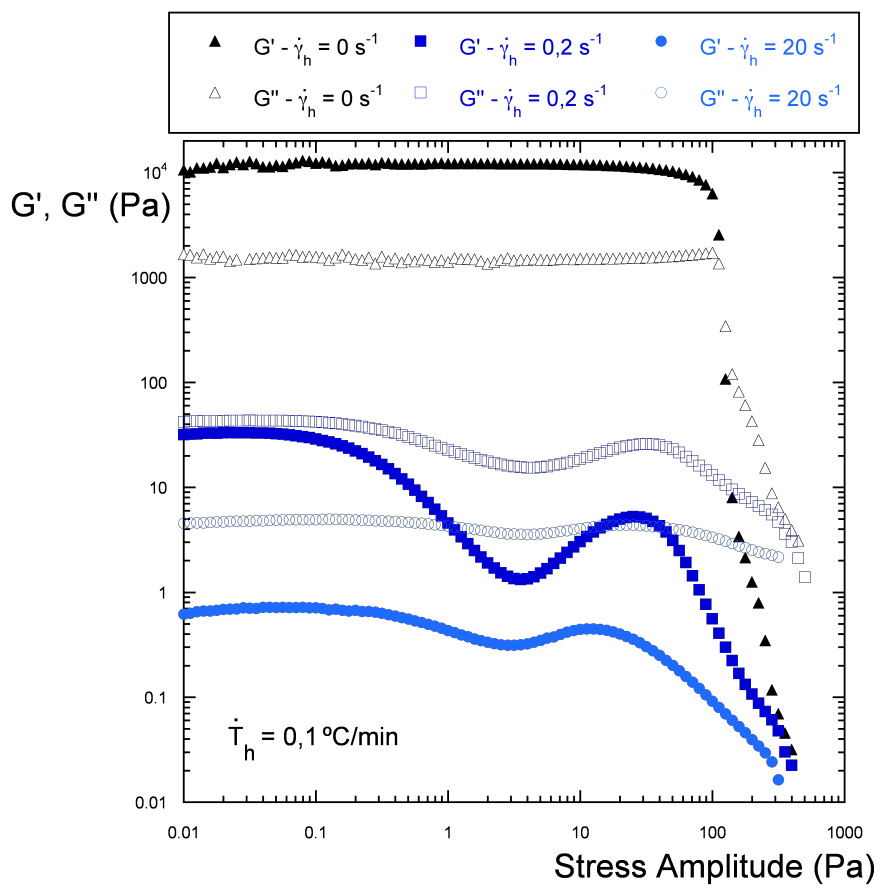

Figure 4.2: Postcooling stress-amplitude-sweep test started immediately after reaching $T_{0}$ : effects of $\dot{\gamma}_{h} ; \dot{T}_{h}=0.1^{\circ} \mathrm{C} / \mathrm{min}$.

The results of the effects of shear history, expressed by the shear rate during cooling $\dot{\gamma}_{h}$, on the post-cooling flow properties for two different cooling rates, $\dot{T}_{h}=0.1^{\circ} \mathrm{C} / \mathrm{min}$ and $\dot{T}_{h}=1{ }^{\circ} \mathrm{C} / \mathrm{min}$, are shown in Figs. 4.2 and 4.3 respectively.

From these figures, it can be noticed that imposing a shear rate during cooling causes both $G^{\prime}$ and $G^{\prime \prime}$ levels to decline. When high enough $\dot{\gamma}_{h}$ 's are applied for a specific cooling rate, an inversion in the moduli can be observed, i.e. $G^{\prime \prime}$ becomes higher than $G^{\prime}$, as previously found by Webber [58]. It is worth noting that even relatively small $\dot{\gamma}_{h}$ 's can lead to an inversion of the moduli, 


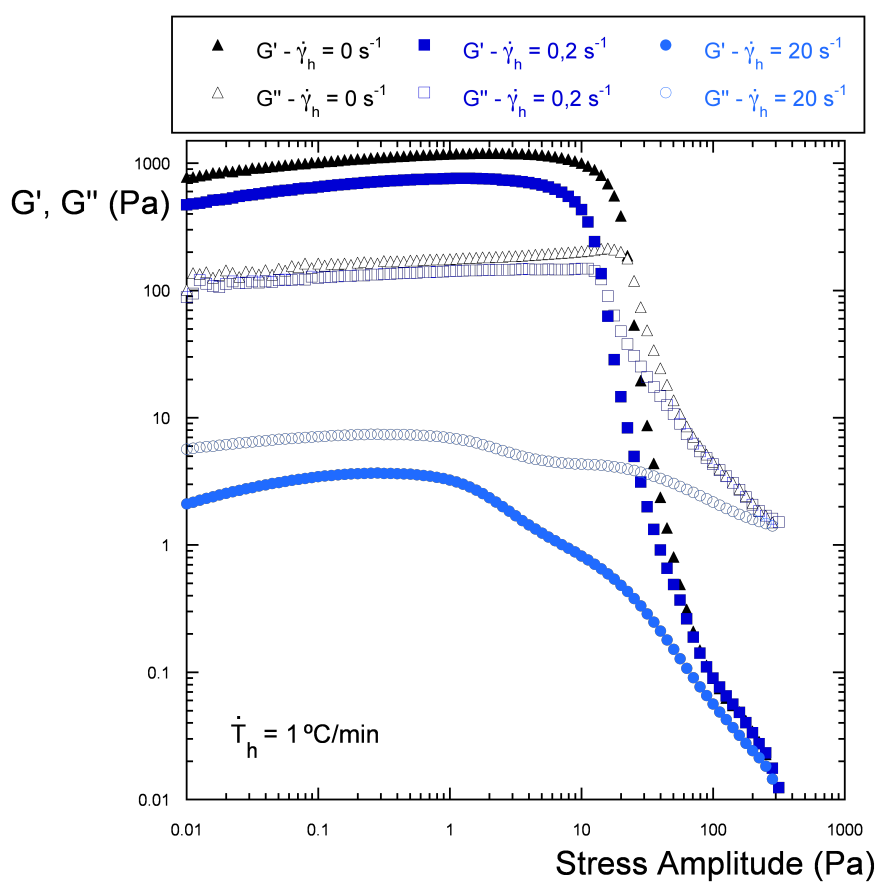

Figure 4.3: Postcooling stress-amplitude-sweep test started immediately after reaching $T_{0}$ : effects of $\dot{\gamma}_{h} ; \dot{T}_{h}=1^{\circ} \mathrm{C} / \mathrm{min}$.

depending on time of shear, which is in fact determined by the cooling rate $\dot{T}_{h}$. In Fig. 4.3, a $\dot{T}_{h}$ of $1^{\circ} \mathrm{C} /$ min means that the cooling process is performed in $46 \mathrm{~min}$. During this time, a $\dot{\gamma}_{h}$ of $0.2 \mathrm{~s}^{-1}$ is not enough to cause a degradation of the microstructure in a way that $G^{\prime \prime}$ becomes higher than $G^{\prime}$. However, in Fig. 4.3, with a ten times smaller cooling rate, which means ten times longer period in which the sample is subjected to shear, the same $\dot{\gamma}_{h}$ causes $G^{\prime \prime}$ to be higher than $G^{\prime}$. Thus, when waxy-oil samples are cooled under shear, it can be observed a degradation of the structure that is more pronounced as the cooling rate $\dot{T}_{h}$ decreases, because decreasing $\dot{T}_{h}$ means increasing the time of shear.

However, when comparing the static cooled samples $\left(\dot{\gamma}_{h}=0 \mathrm{~s}^{-1}\right)$ in both Figs. 4.2 and 4.3, it can be seen that for the lower $\dot{T}_{h}$ (Fig. 4.2), higher $G^{\prime}$ and $G^{\prime \prime}$ levels are achieved, indicating that a stronger structure is formed when low cooling rates are applied. This trend is readily explained by the fact that when longer times are available, wax crystals can become larger and develop stronger interactions between them, giving rise to a stronger structure and higher values of $G^{\prime}$ and $G^{\prime \prime}$.

Thus, in summary, if in one hand a lower $\dot{T}_{h}$ gives rise to larger crystals, stronger structure and higher $G^{\prime}$ and $G^{\prime \prime}$ levels, on the other hand, when the sample is cooled under shear, a lower $\dot{T}_{h}$ leads to a weaker structure and smaller $G^{\prime}$ and $G^{\prime \prime}$ levels because of the longer time of degradation. Even a small $\dot{\gamma}_{h}$ applied for long enough time can break down the waxy structure in a way that 
$G^{\prime \prime}$ becomes higher than $G^{\prime}$, which changes the typical response composed by an elastic region, creep, followed by yielding or fracture. Moreover, in general, small $\dot{\gamma}_{h}$ 's imply more elastic behavior, and also, yielding, when $G^{\prime}>G^{\prime \prime}$. Similar conclusion was drawn by Webber [58] for lubricating mineral oils at low enough temperatures.

After varying the shear rate during cooling $\dot{\gamma}_{h}$ for two different constant cooling rates $\left(\dot{T}_{h}\right.$ 's), we investigated the effects of cooling rate on the results for two different situations: (i) statically cooled samples, which means $\dot{\gamma}_{h}=$ $0 \mathrm{~s}^{-1}$ and (ii) samples cooled under shear, $\dot{\gamma}_{h}=20 \mathrm{~s}^{-1}$. The results for the static cooled samples are presented in Fig. 4.4 and the results for the samples cooled under shear can be seen in Fig. 4.5.

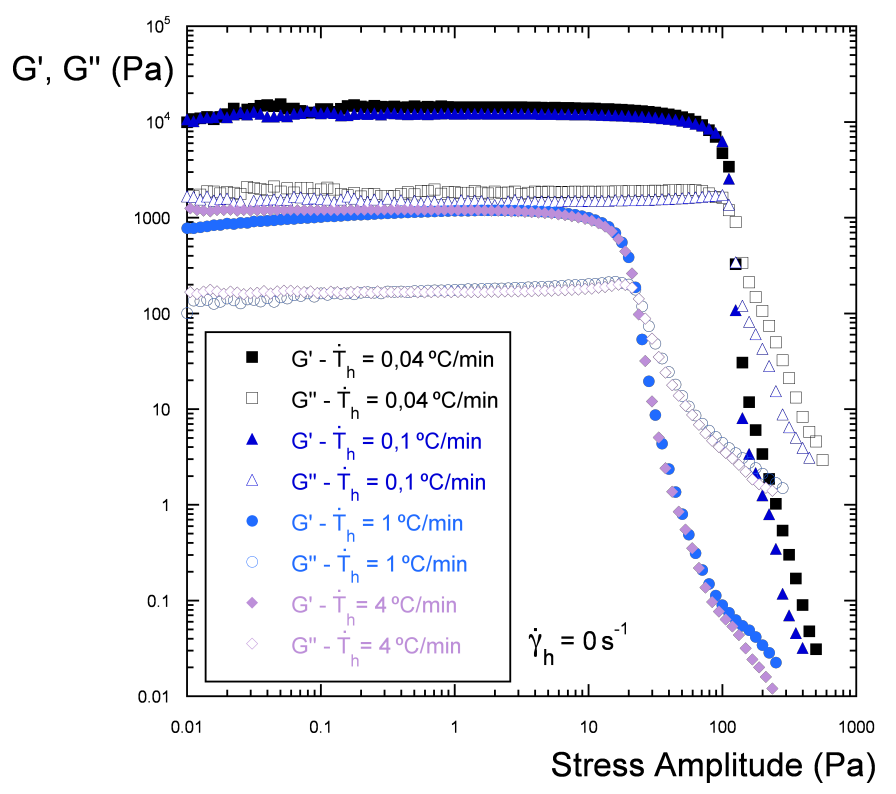

Figure 4.4: Postcooling stress-amplitude-sweep test started immediately after reaching $T_{0}$ : effects of $\dot{T}_{h} ; \dot{\gamma}_{h}=0 \mathrm{~s}^{-1}$.

With regard to the statically cooled samples, it is possible to observe that all stress amplitude sweep tests shown in Fig. 4.4 present qualitatively the same response, composed by elastic region, creep, and then yielding or fracture. Moreover, a large quantitatively difference between the curves pertaining to $\dot{T}_{h}=1{ }^{\circ} \mathrm{C} / \mathrm{min}$ and the curves pertaining to $\dot{T}_{h}=0.1^{\circ} \mathrm{C} / \mathrm{min}$ can be seen. In this range of cooling rates, decreasing $\dot{T}_{h}$ promotes an increase in $G^{\prime}$ and $G^{\prime \prime}$ levels as well as in the yield stress values. As previously mentioned, this fact can be explained as follows: lower cooling rates promote the formation of larger crystals with stronger interaction between then, because of longer times available for crystals growth and structure development. However, it is neither possible to observe a difference between the curves pertaining to the higher cooling rates nor to observe a significant difference between the curves 


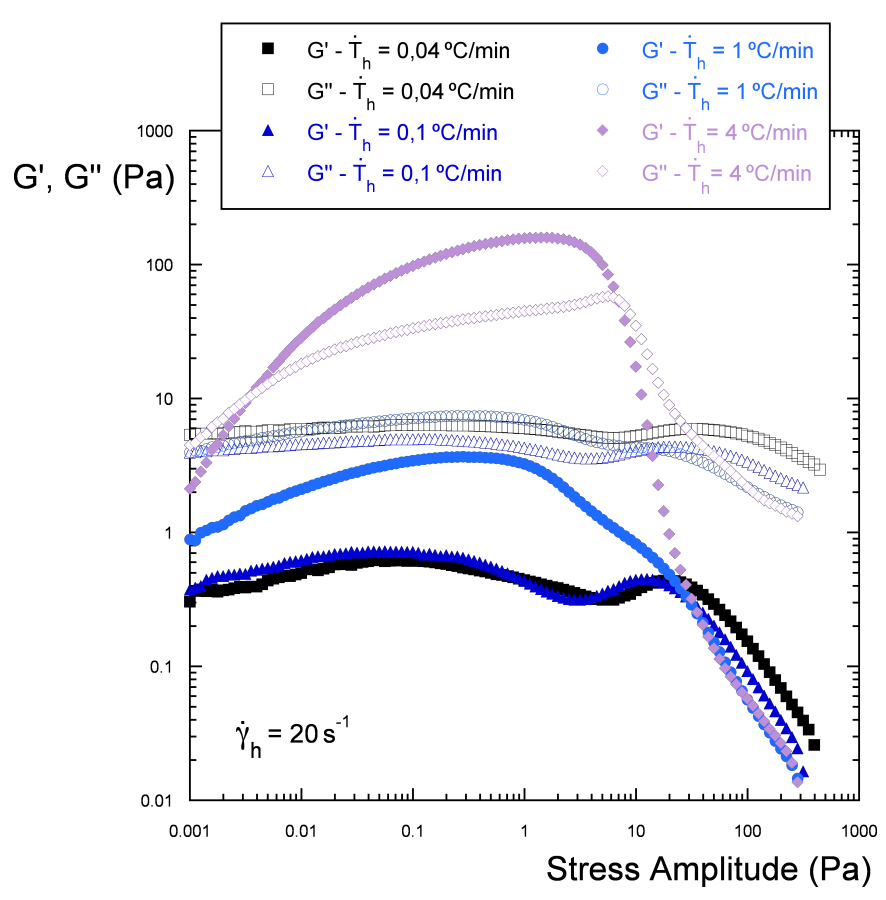

Figure 4.5: Postcooling stress-amplitude-sweep test started immediately after reaching $T_{0}$ : effects of $\dot{T}_{h} ; \dot{\gamma}_{h}=20 \mathrm{~s}^{-1}$.

pertaining to the lower cooling rates. It seems that for cooling rates in the range higher than $1^{\circ} \mathrm{C} / \mathrm{min}$ or lower than $0.1^{\circ} \mathrm{C} / \mathrm{min}$ the wax crystals and structure are not very sensitive to changes in the cooling rate, at least for the Brazilian waxy crude oil and the cooling-rate ranges investigated. Nevertheless, in general, it is possible to state that for statically cooled samples, low cooling rates favor the appearance of larger crystals and stronger structure with higher elasticity and yield stress values.

A different trend is observed for samples exposed to shear during cooling, as yielding and a more elastic behavior can only be seen for high cooling rates, as illustrated in Fig. 4.5. Again, this occurs because $\dot{T}_{h}$ represents the duration of the cooling process, i.e. how long the oil sample will be under shear. Thus, for high $\dot{T}_{h}$ 's, the sample is subjected to shear for short times, so that the degradation of the waxy structure is less pronounced. On the other hand, for lower $\dot{T}_{h}$ 's, the sample is sheared for a longer time, causing more degradation to the waxy structure and, as a consequence, $G^{\prime \prime}$ becomes higher than $G^{\prime}$ and no yielding is observed in this specific test.

In addition, it is possible to see in Fig. 4.5 that in the beginning of the stress amplitude sweep (until $0.005 \mathrm{~Pa}$ ), $G^{\prime \prime}>G^{\prime}$ even for the highest $\dot{T}_{h}$, but, in course of the stress amplitude sweep test, there is a build up of the waxy structure at low stress amplitudes, so that $G^{\prime}$ becomes higher than $G^{\prime \prime}$ and both moduli levels increase during the test. This initial behavior is related to 
the intense shear experienced by the sample during cooling $\left(\dot{\gamma}_{h}=20 \mathrm{~s}^{-1}\right)$ and the absence of a holding time between the cooling process and the oscillatory test.

From this investigation on the influence of the cooling rate on stress amplitude sweep response, it can be concluded that large $\dot{T}_{h}$ 's with shear or small $\dot{T}_{h}$ 's without shear imply more elastic behavior and yielding for this gelled waxy crude oil.

\subsection{3}

\section{Effects of holding time on structure and rheology}

Another important aspect to be investigated is the effects of holding time on the post-cooling flow properties, which is presented in the next set of tests. Two situations were studied: (i) samples that were not submitted to postcooling shear to investigate the effects of holding time before yielding and (ii) samples submitted to post-cooling shear to investigate the effects of holding time after yielding.

\section{Holding time before yielding}

The influence on the results of the holding time before yielding for both samples cooled statically or under shear is shown in Figs. 4.6 and 4.7, respectively. The holding time in Figs. 4.6 and 4.7 is defined as the time after reaching $T_{0}$ and before the beginning of the stress amplitude sweep test.

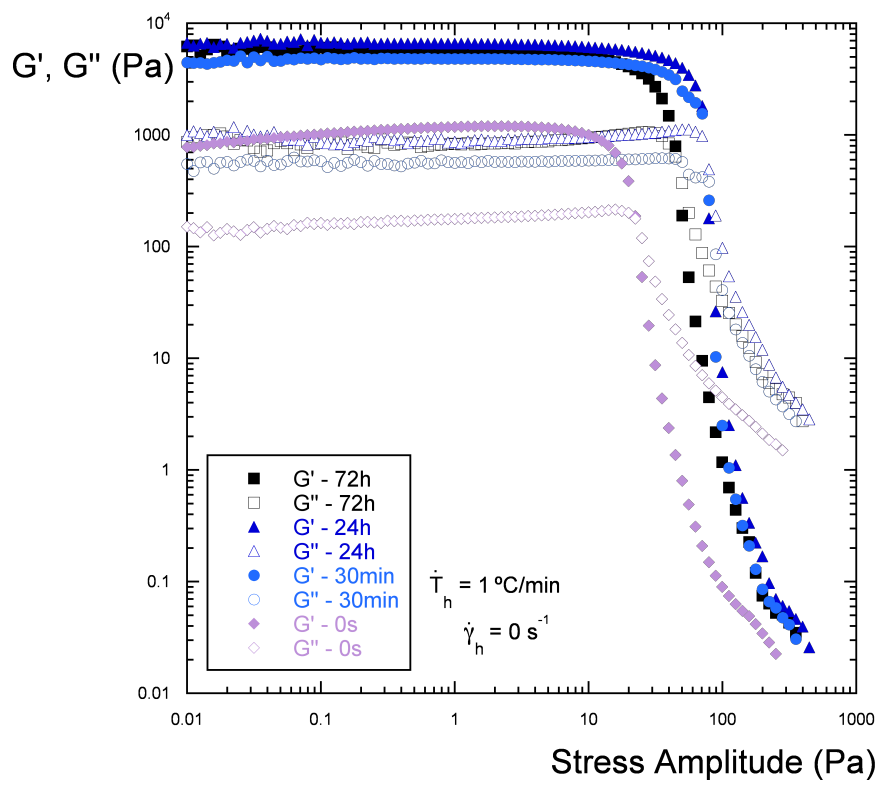

Figure 4.6: Stress-amplitude-sweep test started after reaching $T_{0}$ without postcooling shear: effects of holding time before yielding; $\dot{\gamma}_{h}=0 \mathrm{~s}^{-1}$ and $\dot{T}_{h}=$ $1^{\circ} \mathrm{C} / \mathrm{min}$. 
It can be seen from Fig. 4.6 that, as previously observed, the three regions of a typical response, namely, elastic region, creep, and yielding can be identified in all the tests performed with statically cooled samples. In addition, in all these tests, the gelled waxy oil presents a structuration level of the structure that can be evaluated by the levels of $G^{\prime}$ and $G^{\prime \prime}$. In particular, in the test performed immediately after cooling the sample, which corresponds to the 0 s holding-time test, $G^{\prime}$ is slightly higher than $1000 \mathrm{~Pa}$ in the elastic region. As we increase the holding time before the beginning of the test, $G^{\prime}$ and $G^{\prime \prime}$ increase, which means that the structuration level also increases. However, after 30 min of holding time, no significant difference can be observed in the levels of $G^{\prime}$ and $G^{\prime \prime}$. Thus, a $30 \mathrm{~min}$ holding time before yielding seems to be enough for this gelled waxy oil to reach its maximum structuration level for statically cooled samples with $\dot{T}_{h}=1{ }^{\circ} \mathrm{C} / \mathrm{min}$.

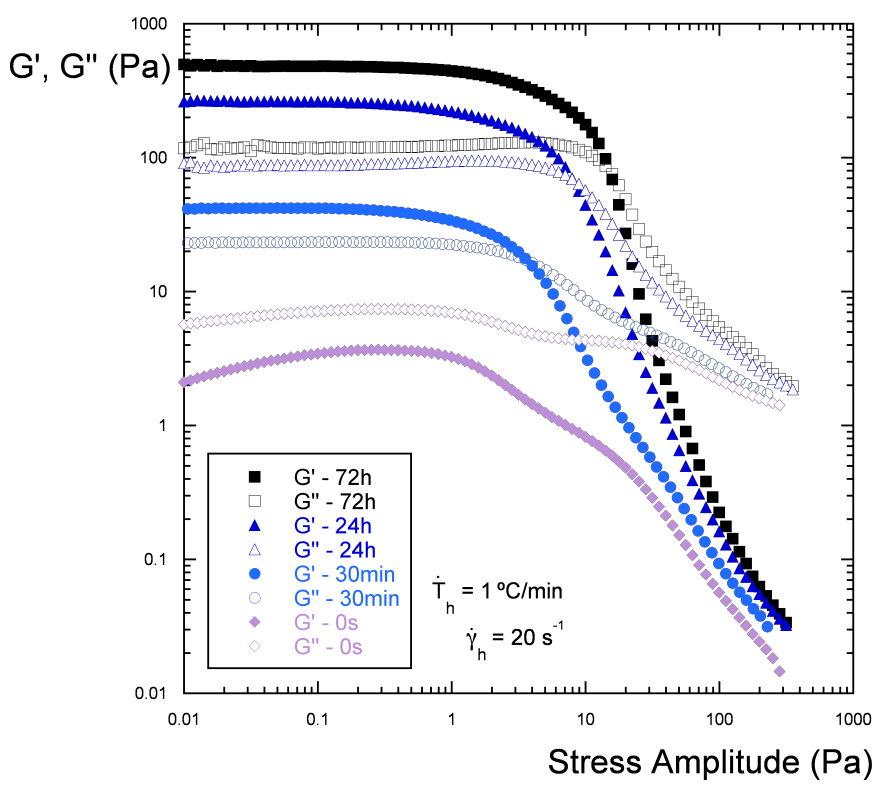

Figure 4.7: Stress-amplitude-sweep test started after reaching $T_{0}$ without postcooling shear: effects of holding time before yielding; $\dot{\gamma}_{h}=20 \mathrm{~s}^{-1}$ and $\dot{T}_{h}=$ $1{ }^{\circ} \mathrm{C} / \mathrm{min}$.

With regard to the tests performed with samples cooled under shear that were not submitted to post-cooling shear, important features in the results can be highlighted. First, it is possible to note in Fig. 4.7 that, unlike the previous result, for this case, in the 0 s holding-time test, $G^{\prime \prime}$ is higher than $G^{\prime}$ during all the test. This fact reflects the low elasticity level of the structure in this case, and also, the absence of a typical response composed by the three clear regions, namely, elastic region, creep, and yielding or fracture. Moreover, by analyzing the curves pertaining to higher holding times, it is clear that the microstructure builds up as the holding time increase because the levels of $G^{\prime}$ 
and $G^{\prime \prime}$ also increase. With 30 min of holding time, $G^{\prime}$ is already higher than $G^{\prime \prime}$, and even after $72 \mathrm{~h}$ of holding time perhaps the structure is still building up. Nevertheless, it is important to point out that the material structuration rate is much higher in the first $24 \mathrm{~h}$, period in which $G^{\prime}$ and $G^{\prime \prime}$ levels increase fast, so that it is possible to conclude that the structuration rate decreases with the holding time. Therefore, it seems that the maximum structuration level of this material for the given $\dot{\gamma}_{h}$ and $\dot{T}_{h}$ is being achieved after $24 \mathrm{~h}$ and would not be much higher than the one achieved after $72 \mathrm{~h}$ of holding time. Actually, perhaps the maximum structuration level is the one corresponded to the $72 \mathrm{~h}$ of holding time.

Thus, in summary, we have seen that the holding time before yielding does make a difference on the post-cooling flow properties for both samples cooled statically or under shear, and is more relevant when the Brazilian waxy crude oil is cooled under shear. Moreover, we can observe that the maximum structuration level achieved by the statically cooled samples is much higher than the maximum structuration level achieved by the samples cooled under shear, which indicates that the shear rate during cooling has irreversible consequences to the gelled waxy structure.

\section{Holding time after yielding}

The influence on the results of the holding time after yielding was investigated by performing stress amplitude sweep tests after applying a $30 \mathrm{~min}$ of post-cooling shear at $20 \mathrm{~s}^{-1}$. The results for samples cooled statically or under shear with $\dot{T}_{h}=1{ }^{\circ} \mathrm{C} / \mathrm{min}$ are plotted in Figs. 4.8 and 4.9 . In these figures the holding time is defined as the time after the $30 \mathrm{~min}$ of post-cooling shear and before the beginning of the stress amplitude sweep test. The only difference between these experiments and the tests shown in Figs. 4.6 and 4.7 is the post-cooling shear.

With regard to the statically cooled samples it is possible to observe in Fig. 4.8 that the results pertaining to the $0 \mathrm{~s}$ of holding time show $G^{\prime \prime}$ higher than $G^{\prime}$ during all the test. In addition, similar to the results presented in Fig. 4.7, the levels of $G^{\prime}$ and $G^{\prime \prime}$ increase with the holding time and after a certain amount of time, $24 \mathrm{~h}$ in this case, $G^{\prime}$ becomes higher than $G^{\prime \prime}$. As a consequence, after $24 \mathrm{~h}$ the elastic region, creep, and yielding can be identified. Moreover, it can be seen in Fig. 4.8 that the structuration rate is also higher in the first $24 \mathrm{~h}$, as previously observed in Fig. 4.7, meaning that after this time the structure is achieving the highest possible structuration level for this thermal and shear history.

By comparing the results of Fig. 4.8 with Fig. 4.6, an interesting analysis 


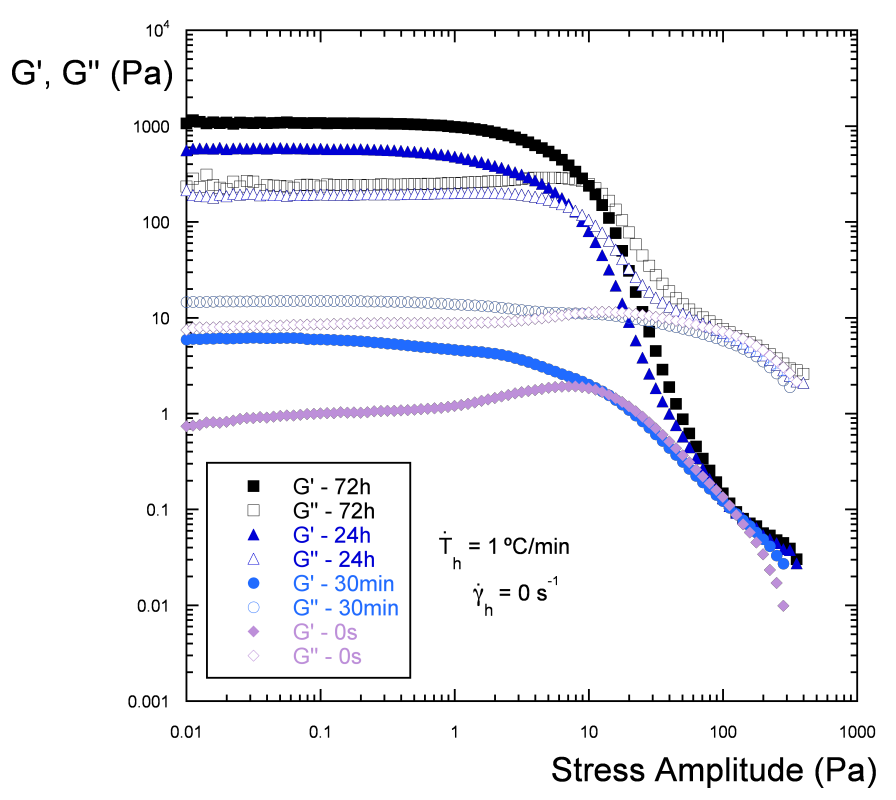

Figure 4.8: Stress-amplitude-sweep test started after $30 \mathrm{~min}$ of postcooling shear at $20 \mathrm{~s}^{-1}$ : effects of holding time; $\dot{\gamma}_{h}=0 \mathrm{~s}^{-1}$ and $\dot{T}_{h}=1{ }^{\circ} \mathrm{C} / \mathrm{min}$.

can be done because the tests in both figures were performed with the same $\dot{\gamma}_{h}$ and $\dot{T}_{h}$. It can be observed in Fig. 4.6 that after 30 min of holding time before yielding, the maximum structuration level of the structure is already achieved for the given $\dot{\gamma}_{h}$ and $\dot{T}_{h}$. In Fig. 4.8 it was possible to observe that, in this case, the maximum structuration level of the structure for the same $\dot{\gamma}_{h}$ and $\dot{T}_{h}$ is being achieved after $24 \mathrm{~h}$ of holding time after yielding and is not much higher than the level obtained after $72 \mathrm{~h}$. However, even after $72 \mathrm{~h}$ of holding time after yielding, the structuration level of the structure is considerable lower than the maximum structuration level achieved by the samples that were not submitted to post-cooling shear. Thus, it is possible to conclude that the shear rate applied after cooling has also irreversible consequences to the waxy structure. Besides that, it is clear that the holding time after yielding also makes difference in the post-cooling flow properties of the statically cooled samples tested.

The results pertaining to the samples cooled under shear are plotted in Fig. 4.9. From this figure it can also be seen that $G^{\prime}$ and $G^{\prime \prime}$ increase with the holding time, and as previously observed for statically cooled samples, $G^{\prime}$ becomes higher than $G^{\prime \prime}$ only after $24 \mathrm{~h}$ of holding time after yielding. Again, it is also possible to observe that the structuration rate of the structure is higher in the first $24 \mathrm{~h}$, so that after this time the structure is achieving the maximum structuration level, that is expected not to be much higher than the one achieved after $72 \mathrm{~h}$.

Similar conclusions to the ones obtained for statically cooled samples 


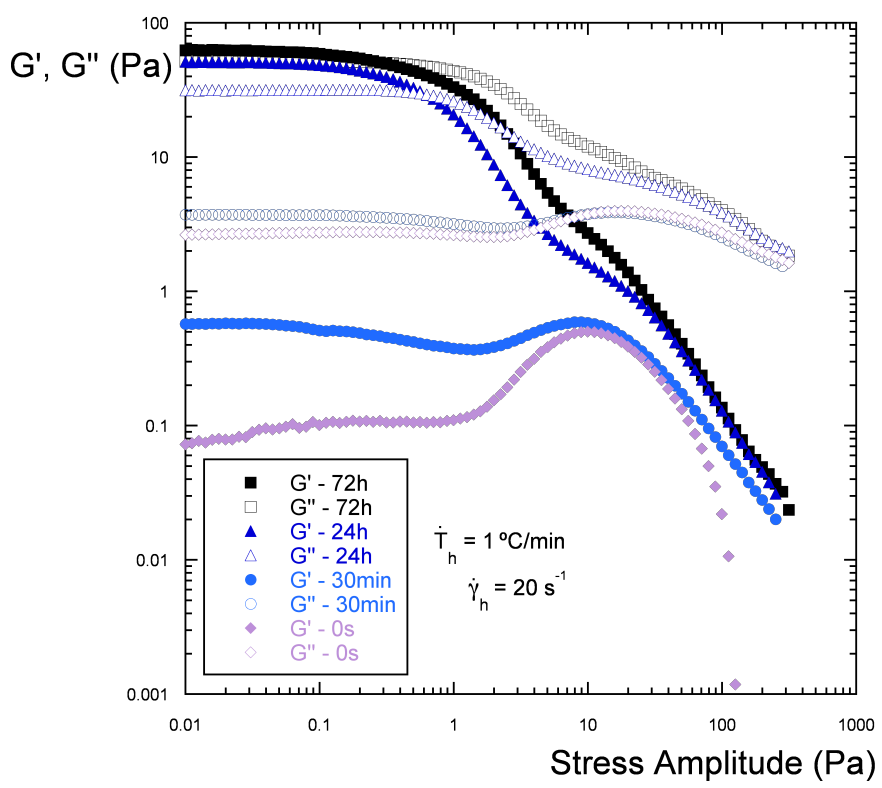

Figure 4.9: Stress-amplitude-sweep test started after $30 \mathrm{~min}$ of postcooling shear at $20 \mathrm{~s}^{-1}$ : effects of holding time; $\dot{\gamma}_{h}=20 \mathrm{~s}^{-1}$ and $\dot{T}_{h}=1{ }^{\circ} \mathrm{C} / \mathrm{min}$.

can be drawn for the samples cooled under shear. By comparing Fig. 4.9 with Fig. 4.7 it is possible to observe that the maximum structuration level of the structure for the samples submitted to post-cooling shear is much lower than the maximum level obtained with samples that were not submitted to postcooling shear, for the same applied $\dot{\gamma}_{h}$ and $\dot{T}_{h}$. Thus, the post-cooling shear has also irreversible consequences to the samples cooled under shear.

\section{Overview}

From the results and discussion presented above with regard to the effects of holding time on waxy structure and rheology, it is possible to conclude that for both samples cooled statically or under shear: (i) the holding time both before and after yielding does make a difference on oil rheology, (ii) the structuration rate of the structure decreases with the holding time both before and after yielding, and (iii) the structure can build up during the holding time both before and after yielding, but the shear rate both during and after cooling has irreversible consequences to the structure.

\subsection{4}

\section{Effects of frequency on structure and rheology}

One last important investigation presented in this chapter is the effects on the results of changing the frequency. Stress amplitude sweep test results obtained with different frequencies for both samples cooled statically or under shear are shown in Figs. 4.10 and 4.11, respectively. 


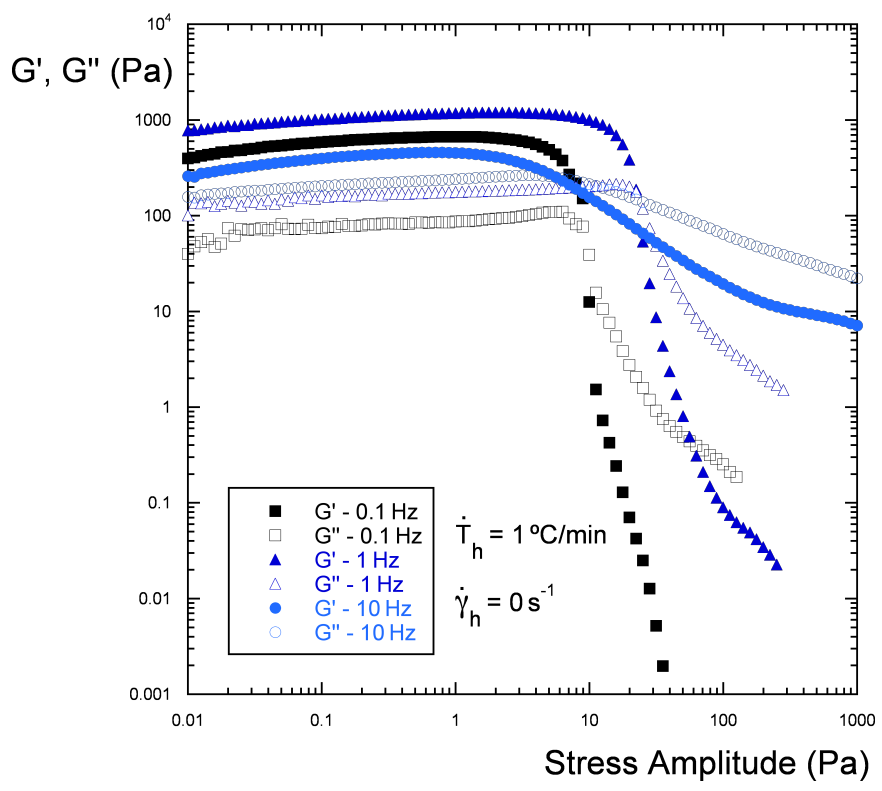

Figure 4.10: Postcooling stress-amplitude-sweep test started immediately after reaching $T_{0}$ : effects of frequency; $\dot{\gamma}_{h}=0 \mathrm{~s}^{-1}$ and $\dot{T}_{h}=1{ }^{\circ} \mathrm{C} / \mathrm{min}$.

From Fig. 4.10 it can be observed that $G^{\prime}$ and $G^{\prime \prime}$ levels pertaining to the test performed at $1 \mathrm{~Hz}$ are higher than the levels of $G^{\prime}$ and $G^{\prime \prime}$ pertaining to the test performed at $0.1 \mathrm{~Hz}$. This observed trend indicates that increasing the frequency in this range of frequencies, which means decreasing the time of observation, lead us to obtain high measurements of $G^{\prime}, G^{\prime \prime}$, and elastic and static yield stresses. Chang et al. [52] observed the same trend for statically cooled samples with regard to $G^{\prime}, G^{\prime \prime}$, and static yield stress but observed that the elastic yield stress is independent of the frequency. They interpreted this result as a time-dependency of the static yield stress.

However, in the same Fig. 4.10, increasing the frequency from 1 to $10 \mathrm{~Hz}$ causes a decrease in $G^{\prime}, G^{\prime \prime}$, and elastic- and static-yield-stress measurements. Again, Chang et al. [52] observed the same trend for statically cooled samples, except that the elastic yield stress measured by them was independent of the frequency. They explained the decrease of $G^{\prime}, G^{\prime \prime}$, and static yield stress with an increase in the frequency by arguing that a degradation of the structure is caused by high frequencies, resulting in lower levels of $G^{\prime}, G^{\prime \prime}$, and static yield stress.

With regard to effects of frequency on the results obtained with samples cooled under shear, in Fig. 4.11 we can observe that in the beginning of the tests, $G^{\prime \prime}$ is higher than $G^{\prime}$ for all frequencies tested. In addition, we observe a significant increase in $G^{\prime}$ and $G^{\prime \prime}$ levels in the low stress-amplitude range for the tests performed at 0.1 and $1 \mathrm{~Hz}$, in a way that, after a certain value of stress amplitude, $G^{\prime}$ becomes higher than $G^{\prime \prime}$. This observation reflects a 
build up of the structure in this range of stress amplitude. Moreover, if we take the maximum in $G^{\prime}$ as a measurement of the elastic yield stress in the tests performed at 0.1 and $1 \mathrm{~Hz}$, it is not possible to observe a significant difference between both, which can be an indication that the elastic yield stress measurements at low frequencies do not depend on the frequency for samples cooled under shear.

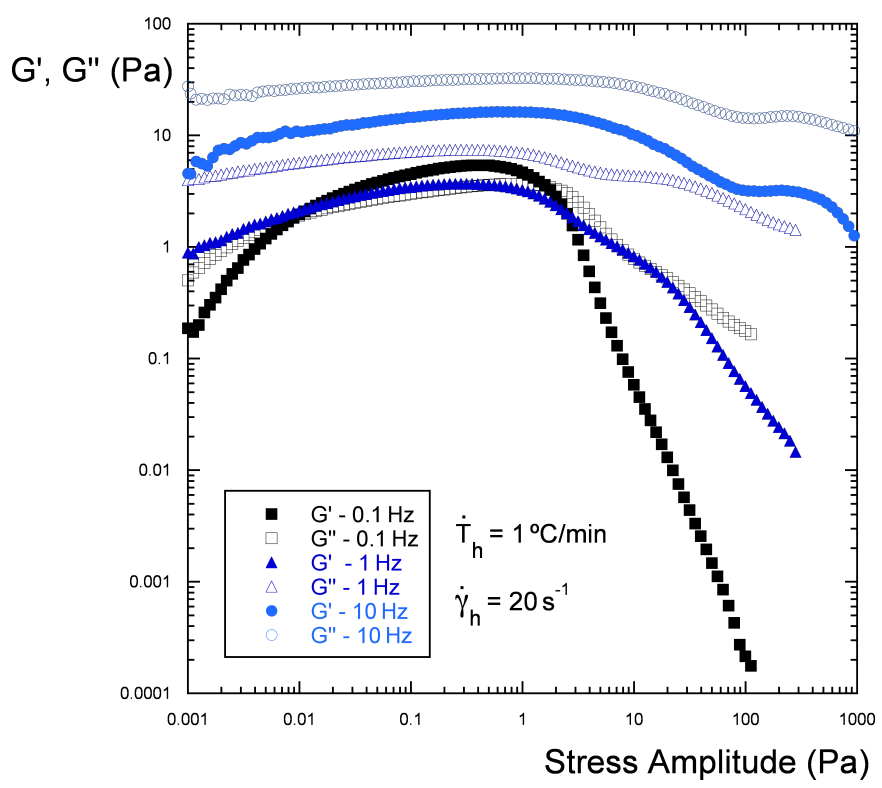

Figure 4.11: Postcooling stress-amplitude-sweep test started immediately after reaching $T_{0}$ : effects of frequency; $\dot{\gamma}_{h}=20 \mathrm{~s}^{-1}$ and $\dot{T}_{h}=1{ }^{\circ} \mathrm{C} / \mathrm{min}$.

A different trend is observed in Fig. 4.11 in the test performed at $10 \mathrm{~Hz}$, that is $G^{\prime \prime}$ is higher than $G^{\prime}$ during all the test. Similar to the results found by Chang et al. [52] for statically cooled samples, it is possible that $10 \mathrm{~Hz}$ is a high enough frequency to cause a significant degradation of the structure, which avoid a sufficiently build up of the structure to cause $G^{\prime}$ to become higher than $G^{\prime \prime}$. 


\section{4}

\section{Final remarks}

In this chapter we presented post-cooling stress amplitude sweep results for the Brazilian waxy crude oil investigated. We studied the yielding and also the effects of thermal and shear histories, the effects of holding time before and after yielding, and the effects of changing the frequency on the results.

The stress amplitude sweep test is a convenient way to investigate the post-cooling rheological behavior of waxy crude oils because of its simplicity and relatively short time required to evaluate both the elastic and static yield stresses. However, a comparison between the results presented in this chapter and the results presented in Chapter 3 remains to be done to evaluate the reliability of the measurements.

With respect to the effects of thermal and shear histories on waxy structure and post-cooling rheology we observe that, on one hand, for statically cooled samples $\left(\dot{\gamma}_{h}=0\right)$, decreasing the cooling rate $\dot{T}_{h}$ causes an increase in elasticity, $G^{\prime}, G^{\prime \prime}$, and both elastic and static yield stresses. This trend is explained by the fact that decreasing $\dot{T}_{h}$ gives more time for crystals growth and structure development. Moreover, in this case that $\dot{\gamma}_{h}=0$, we always observe in the tests the three typical regions, namely, elastic region, creep, and yielding or fracture.

On the other hand, for samples cooled under shear $\left(\dot{\gamma}_{h}>0\right)$, increasing $\dot{T}_{h}$ causes an increase in elasticity, $G^{\prime}, G^{\prime \prime}$, and elastic and static yield stresses in the case that $G^{\prime}>G^{\prime \prime}$ in the low stress-amplitude range. When $\dot{\gamma}_{h}>0$, the three typical regions are only observed in stress amplitude sweep results for high enough $\dot{T}_{h}$ 's, because increasing the cooling rate reduces the time along which the sample is submitted to shear, causing less degradation to the waxy structure. We also observe that even small $\dot{\gamma}_{h}$ 's applied for long enough times can cause a degradation of the structure in a way that $G^{\prime \prime}$ becomes higher than $G^{\prime}$ during all the test.

Another investigation was performed to understand the effects of holding time both before and after yielding on waxy structure and post-cooling rheology. We observe that for both samples cooled under shear or statically the holding time before and after yielding does make difference. The structure builds up during both the holding time before and after yielding, but the shear rate applied during and/or after cooling has irreversible consequences to the waxy structure and rheology. Only by thermal cycling that the original structure and rheology can be recovered.

With regard to the effects of frequency on the results, we observe that for both samples cooled under shear or statically, high enough frequencies 
cause a degradation of the structure, thus affecting the measurements of the yield stresses. For statically cooled samples and low enough frequencies it is possible that the elastic yield stress measurements become independent of the frequency, as previously found by Chang et al. [52], but additional investigation is required. 


\section{5 \\ Concluding Remarks}

In this thesis, we investigated the rheology of waxy crude oils. The main motivation behind this study is the increasing necessity of transporting waxy crudes through pipelines at low temperatures. This task becomes particularly difficult after a long period of shutdown, because the crystallization of wax leads to a gelation process and consequently to an extremely complex rheological behavior.

Above the Wax Appearance Temperature $W A T$, waxy oils are known to present a Newtonian behavior, with an Arrhenius-temperature dependence of the viscosity. However, at low enough temperatures, waxy crude oils present a rheological behavior that is a function not only on the temperature, shear rate, and time of shear, but also on the thermal and shear histories experienced by the oil.

Because of this complex behavior, a number of precautions are required to carry out the rheological characterization of a given waxy crude oil. We described in Chapter 2 an experimental protocol, based on the relevant literature, designed to ensure well defined thermal and shear histories of a waxy crude oil sample to be submitted to rheological measurements. We showed that precautions, in addition to the precautions recommended in the literature, must be taken during the preparation of the sample of a waxy crude oil to obtain meaningful rheological data, especially in the case of transient measurements.

This protocol is composed by three main steps, namely, (i) the pretreatment, (ii) an isothermal holding time, and (iii) the cooling process. The pretreatment consists on imposing heating to the waxy crude oil to guarantee a stable chemical composition. During this step, the oil should not be exposed to temperatures that differ from the temperatures observed in the field application to avoid effects of resins and asphaltenes on oil rheology that would not be observed in the field. The second step, an isothermal holding time at the starting temperature, is performed with the appropriate geometry and gap to be used in rheological measurements to dissolve all wax crystals and erase the thermal memory of the oil. The third step, the cooling process, must also be 
carried out with the appropriate geometry and gap and imposes to the sample well defined thermal and shear histories. The test conditions, that include the starting temperature, geometry, and gap are obtained before the steps (ii) and (iii) with the aid of thermal cycle tests, performed according to the procedures described in Chapter 2.

After the cooling process is carried out and the measurement temperature is achieved, the post-cooling oil rheology can be investigated. In this thesis we obtained a fair complete rheological characterization of a Brazilian waxy crude oil, by conducting four rheometric experiments, namely, steady-state flow, constant shear rate, constant shear stress, and dynamic-stress-amplitude sweep with both samples cooled under shear or statically.

We evaluated the yield stresses of the Brazilian waxy crude oil from two techniques in Chapter 3, the bifurcation plot of strain or shear rate, obtained from constant shear stress tests, and the extrapolation of steady-state data to the limit of zero shear rate in a flow curve. In this last case, the steady-state results were confirmed by performing constant shear rate tests and evaluating the minimum time above which the results stop varying with time.

On the one hand, for samples cooled under shear, we observed a reasonably good agreement between the yield stresses obtained from the two techniques, indicating that one single yield stress exist in this case. On the other hand, for statically cooled samples, we observed that the yield stress obtained from bifurcation results is roughly three times higher than the extrapolated yield stress in a flow curve. We also observed that for high enough shear rates, the steady-state result does not depend significantly on the previous history experienced. Therefore, we highlighted the possibility of the existence of two yield stresses and a non-monotonic flow curve when the samples are statically cooled.

With regard to the stress amplitude sweep tests presented in Chapter 4, it is important to point out that in many situations the test was performed just after achieving the measurement temperature and sometimes the periodic steady state was not achieved. Even though it happened, important features in gelled oil rheology could be observed and different conclusions can be drawn.

By performing these oscillatory tests, we studied the yielding and also the effects of thermal and shear histories, the effects of holding time before and after yielding, and the effects of changing the frequency on the results.

On the one hand, for statically cooled samples, the three typical regions, namely, elastic region, creep, and yielding or fracture could be observed in all cases. Moreover, we observed that decreasing the cooling rate $\dot{T}_{h}$ causes an increase in elasticity and yield stress values. This trend can be readily explained 
by the fact that decreasing the cooling rate gives more time to crystals growth and structure development. On the other hand, for samples cooled under shear, the three typical regions, including yielding, could be observed only for high cooling rate values. In addition, decreasing the cooling rate $\dot{T}_{h}$ causes a decrease in elasticity and yield stress values. This opposite trend is explained by the fact that decreasing $\dot{T}_{h}$, in this case, causes the time of shear to increase, which lead to more degradation of the waxy structure.

We also investigated the effects of holding time on oil rheology. We observed that for both samples cooled under shear or statically the holding time before and after yielding does make difference on oil rheology. The structure builds up during both the holding times before and after yielding, but the shear rate applied during and/or after cooling has irreversible consequences to the waxy structure and rheology. Only by thermal cycling that the original structure and rheology can be recovered.

At last, with regard to the effects of changing the frequency on the results, we observed that for both samples cooled under shear or statically, high enough frequencies cause a degradation of the structure, thus affecting the measurements of the yield stresses. For low enough frequencies we highlighted the possibility that the elastic yield stress measurements become independent of the frequency, as previously found by Chang et al. [52], but additional investigation is required to confirm this find.

\section{1}

Suggestions for future research works

As pointed out in Chapter 3, three important topics are being recurrent themes of investigation by different research groups, (i) the rheological characterization of gelled waxy oils, (ii) constitutive equations to describe the extremely complex behavior of these materials, and (iii) mathematical modeling of startup or restart of gelled oil pipelines.

With regard to the theme (i) we observed that there is still no universally accepted protocol to perform the rheological characterization of waxy crude oils. Therefore, we can suggest for future developments to evaluate the performance of the protocol described in Chapter 2 for rheological characterization of different crude oils. In addition, three important investigations related to this topic still remain to be done, (i) the mechanism by which small-gap geometries affect the bulk crystallization process, (ii) how the inhomogeneity inherent to parallel plates and concentric cylinders can affect rheological measurements, and (iii) the importance of apparent wall slip and shear banding to the measurements. 
Besides that, a better understanding of the gelation phenomenon and the characteristic temperatures related to changes in rheological behavior is in order. Moreover, it would be useful to have extensive rheological data from different waxy crude oils associated with microscopic measurements, in particular, data obtained from oils that possess higher wax contents. This can certainly give insight into gelled crude oil rheology and enable the development of more accurate constitutive models. Two important features cannot be appropriately addressed by the existing models, (i) isothermal irreversible shear degradation and (ii) recovery of the structure with thermal cycling. Only after having deeper knowledge about the above suggested topics that a really accurate startup model of gelled oil pipelines can be developed. 


\section{Bibliography}

[1] SMITH, P. B.; RAMSDEN, R. M. J.. The prediction of oil gelation in submarine pipelines and the pressure required for restarting flow. Eur. Offshore Petrol. Conf., p. 283-290, 1978. 1.1, 2.1, 2.3.1, 2.4.5, $2.4 .5,2.5 .3$

[2] LEE, H. S.. Computational and Rheological Study of Wax Deposition and Gelation in Subsea Pipelines. Tese de Doutorado, University of Michigan, 2008. 1.1

[3] RØNNINGSEN, H. P.. Rheological behavior of gelled, waxy north sea crude oils. J. Petrol. Sci. Eng., 7:177-213, 1992. 1.1, 2.1, 2.4.3, $2.5 .4,3.2$

[4] DE SOUZA MENDES, P. R.; BRAGA, S. L.. Obstruction of pipelines during the flow of waxy crude oils. Journal of Fluids EngineeringTransactions of the ASME, 118(4):722-728, 1996. 1.1

[5] DE SOUZA MENDES, P. R.; BRAGA, A. M. B.; AZEVEDO, L. F. A. ; CORREA, K. S.. Resistive force of wax deposits during pigging operations. Journal of Energy Resourses Technology-Transactions of the ASME, 121(3):167-171, 1999. 1.1

[6] VENKATESAN, R.; NAGARAJAN, N. R.; PASO, K.; YI, Y. B.; SASTRY, A. M. ; FOGLER, H. S.. The strength of paraffin gels formed under static and flowconditions. Chem. Eng. Sci., 60:3587-3598, 2005. 1.1, $2.1,2.4 .3,2.4 .5$

[7] BURGER, E. D.; PERKINS, T. K. ; STRIEGLER, J. H.. Studies of wax deposition in the trans-alaska pipeline. J. Petrol. Tech., 33:10751086, 1981. 1.1

[8] MAJEED, A.; BRINGEDAL, B. ; OVERA, S.. Model calculates wax deposition for n. sea oils. Oil and Gas J., 88:63-69, 1990. 1.1

[9] SVENDSEN, J. A.. Mathematical-modeling of wax deposition in oil pipeline systems. AIChE J., 39(8):1377-1388, 1993. 1.1 
[10] RIBEIRO, F. S.; DE SOUZA MENDES, P. R. ; BRAGA, S. L.. Obstruction of pipelines due to paraffin deposition during the flow of crude oils. Int. J. Heat Mass Transfer., 40(18):4319-4328, 1997. 1.1

[11] COUTINHO, J. A. P.. Predictive uniquac: A new model for the description of multiphase solid-liquid equilibria in complex hydrocarbon mixtures. Ind. Eng. Chem. Res., 37(12):4870-4875, 1998. 1.1

[12] SINGH, P.; FOGLER, H. S. ; NAGARAJAN, N.. Prediction of the wax content of the incipient wax-oil gel in a pipeline: An application of the controlled-stress rheometer. J. Rheol., 43(6):1437-1459, November/December 1999. 1.1, 2.1, 2.4.3, 2.5.5

[13] SINGH, P.; VENKATESAN, R.; FOGLER, H. S. ; NAGARAJAN, N.. Formation and aging of incipient thin film wax-oil gels. AIChE Journal, 46(5):1059-1074, 2000. 1.1

[14] SINGH, P.; VENKATESAN, R.; FOGLER, H. S. ; NAGARAJAN, N. R.. Morphological evolution of thick wax deposits during aging. AIChE Journal, 47(1):6-18, 2001. 1.1

[15] SINGH, P.; YOUYEN, A. ; FOGLER, H. S.. Existence of a critical carbon number in the aging of a wax-oil gel. AIChE Journal, 47(9):2111-2124, 2001. 1.1

[16] KOK, M. V.; SARACOGLU, R. O.. Mathematical modelling of wax deposition in crude oil pipelines (comparative study). Petrol. Sci. Tech., 18(9-10):1121-1145, 2000. 1.1

[17] AZEVEDO, L. F. A.; TEIXEIRA, A. M.. A critical review of the modeling of wax deposition mechanisms. Petrol. Sci. Tech., 21(34):393-408, 2003. 1.1

[18] RAMIREZ-JARAMILLO, E.; LIRA-GALEANA, C. ; MANERO, O.. Modeling wax deposition in pipelines. Petrol. Sci. Tech., 22(7-8):821-861, 2004. 1.1

[19] BHAT, N. V.; MEHROTRA, A. K.. Modeling of deposit formation from "waxy" mixtures via moving boundary formulation: Radial heat transfer under static and laminar flow conditions. Ind. Eng. Chem. Res., 44(13):6948-6962, 2005. 1.1 
[20] BHAT, N. V.; MEHROTRA, A. K.. Modeling of deposition from "waxy" mixtures in a pipeline under laminar flow conditions via moving boundary formulation. Ind. Eng. Chem. Res., 45(25):87288737, 2006. 1.1

[21] BHAT, N. V.; MEHROTRA, A. K.. Modeling the effect of shear stress on the composition and growth of the deposit layer from "waxy" mixtures under laminar flow in a pipeline. Energy Fuels, 22:3237-3248, 2008. 1.1

[22] COUTINHO, J. A. P.; EDMONDS, B.; MOORWOOD, T.; SZCZEPANSKI, R. ; ZHANG, X.. Reliable wax predictions for flow assurance. Energy \& Fuels, 20:1081-1088, 2006. 1.1

[23] COUTINHO, J. A. P.; MIRANTE, F. ; PAULY, J.. A new predictive UNIQUAC for modeling of wax formation in hydrocarbon fluids. Fluid Phase Equilibria, 247:8-17, 2006. 1.1

[24] MEHROTRA, A. K.; BHAT, N. V.. Modeling the effect of shear stress on deposition from "waxy" mixtures under laminar flow with heat transfer. Energy \& Fuels, 21(3):1277-1286, 2007. 1.1

[25] MEHROTRA, A. K.; BHAT, N. V.. Deposition from "waxy" mixtures under turbulent flow in pipelines: Inclusion of a viscoplastic deformation model for deposit aging. Energy \& Fuels, 24:2240-2248, 2010. 1.1

[26] TIWARY, R.; MEHROTRA, A. K.. Deposition from wax-solvent mixtures under turbulent flow: Effects of shear rate and time on deposit properties. Energy \& Fuels, 23:1299-1310, 2009. 1.1

[27] BIDMUS, H. O.; MEHROTRA, A. K.. Solids deposition during "cold flow" of wax-solvent mixtures in a flow-loop apparatus with heat transfer. Energy \& Fuels, 23:3184-3194, 2009. 1.1

[28] BOUCETTA, R.; KESSAL, M.. Modelization of the upslope flow of heated petroleum paraffin. Can. J. Chem. Eng., 88(2):190-199, 2010. 1.1

[29] HUANG, Z.; SENRA, M.; KAPOOR, R. ; FOGLER, H. S.. Wax deposition modeling of oil/water stratified channel flow. AIChE J., 57(4):841851, 2011. 1.1 
[30] GAUTIER, D. L.; BIRD, K. J.; CHARPENTIER, R. R.; GRANTZ, A.; HOUSEKNECHT, D. W.; KLETT, T. R.; MOORE, T. E.; PITMAN, J. K.; SCHENK, C. J.; SCHUENEMEYER, J. H.; S $\phi$ RENSEN, K.; TENNYSON, M. E.; VALIN, Z. C. ; WANDREY, C. J.. Assessment of undiscovered oil and gas in the arctic. Science, 324:1175-1179, 2009. 1.1

[31] WEBBER, R. M.. Low temperature rheology of lubricating mineral oils: Effects of cooling rate and wax crystallization on flow properties of base oils. J. Rheol., 43(4):911-931, 1999. 1.1, 2.1, 2.4.2, 2.4.3, 2.4.4, 2.5.3, 2.5.5, 3.4.3, 4.3.1

[32] KANÉ, M.; DJABOUROV, M.; VOLLE, J.-L.; LECHAIRE, J.-P. ; FREBOURG, G.. Morphology of paraffin crystals in waxy crude oils cooled in quiescent conditions and under flow. Fuel, 82:127-135, 2003. 1.1

[33] WARDHAUGH, L. T.; BOGER, D. V.. Measurement of the unique flow properties of waxy crude oils. Chemical Engineering Research \& Design, 65:74-83, 1987. 1.1, 2.1, 2.3.1, 2.3.2, 2.4.3, 2.5.5, 3.2, 3.4.2, 3.4.3

[34] WARDHAUGH, L. T.; BOGER, D. V.. Flow characteristics of waxy crude oils: Application to pipeline design. AIChE Journal, 37(6):871-885, 1991. 1.1, 2.1, 2.3.1, 2.3.2, 2.4.3, 2.5.5, 3.2, 3.4.2, 3.4.3

[35] DAVEnPORT, T. C.; SOMPER, R. S. H.. The yield value and breakdown of crude oil gels. J. Inst. Petrol., 57(554):86-105, 1971. $1.1,2.1,3.2,3.4 .3$

[36] MARCHESINI, F. H.; ALICKE, A. A.; DE SOUZA MENDES, P. R. ; ZÍGLIO, C. M.. Rheological characterization of waxy crude oils: Sample preparation. Energy Fuels, 26(5):2566-2577, 2012. 2, 3.1, 3.3, 4.2

[37] CHANG, C.; NGUYEN, Q. D. ; RØNNINGSEN, H. P.. Isothermal startup of pipeline transporting waxy crude oil. J. Non-Newtonian Fluid Mech., 87:127-154, 1999. 2.1, 4.3.1

[38] PERKINS, T. K.; TURNER, J. B.. Starting behavior of gathering lines and pipelines filled with gelled prudhoe bay oil. J. Petrol. Tech., 23:301-\&, 1971. 2.1

[39] BARRY, E. G.. Pumping non-newtonian waxy crude oils. J. Inst. Petrol., 57:74-\&, 1971. 2.1 
[40] RUSSELL, R. J.; CHAPMAN, E. D.. The pumping of 85 degrees $\mathbf{f}$ pour point assam (nahorkatiya) crude oil at 65 degrees $\mathrm{f}$. J. Inst. Petrol., 57:117-\&, 1971. 2.1

[41] VERSCHUUR, E.; VERHEUL, C. M. ; HARTOG, A. P. D.. The effect of thermal shrinkage and compressibility on the yielding of gelled waxy crude oils in pipelines. J. Inst. Pet., 57(555):131-138, 1971. 2.1

[42] VERSCHUUR, E.; VERHEUL, C. M. ; HARTOG, A. P. D.. Pilot-scale studies on re-starting pipelines containing gelled waxy crudes. J. Inst. Pet., 57(555):139-146, 1971. 2.1

[43] GOVIER, G. W.; FOGARASI, M.. The interpretation of data on the rheological behaviour of thixotropic crude oils. J. Can. Pet. Technol., p. 42-48, 1972. 2.1

[44] PETRELLIS, N. C.; FLUMERFELT, R. W.. Rheological behaviour of shear degradable oils: Kinetic and equilibrium properties. Can. J. Chem. Eng., 151:291-301, 1973. 2.1, 2.3.1, 3.2

[45] SIFFERMAN, T. R.. Flow properties of difficult-to-handle waxy crude oils. J. Petrol. Tech., p. 1042-1050, 1979. 2.1

[46] WARDHAUGH, L. T.; BOGER, D. V.. The measurement and description of the yielding behavior of waxy crude oil. J. Rheol., 35(6):1121-1156, 1991. 2.1, 2.4.3, 2.5.4, 2.5.5, 3.2

[47] FORD, P. E.; ELLS, J. W. ; RUSSELL, R. J.. Pipelining high-pourpoint crude. part. 1: What troubles can be anticipated - how to meet them? Oil and Gas J., p. 88-91, April 19 1965. 2.1

[48] FORD, P. E.; ELLS, J. W. ; RUSSELL, R. J.. Pipelining high-pourpoint crude. part. 2. Oil and Gas J., p. 107-109, April 26 1965. 2.1

[49] FORD, P. E.; ELLS, J. W. ; RUSSELL, R. J.. Pipelining high-pourpoint crude. part. 3: Frequent pigging helps move waxy crude below its pour point. Oil and Gas J., p. 183-189, May 10 1965. 2.1

[50] FORD, P. E.; ELLS, J. W. ; RUSSELL, R. J.. Pipelining high-pourpoint crude. part. 4: What pressure is required for restarting gelled line? Oil and Gas J., p. 134-136, May 17 1965. 2.1

[51] CAWKWELL, M. G.; CHARLES, M. E.. Characterization of canadian artic thixotropic gelled crude oils utilizing an eight-parameter model. J. Pipelines, 7:251-264, 1989. 2.1, 2.3.2, 2.4 .3 
[52] CHANG, C.; BOGER, D. V. ; NGUYEN, Q. D.. The yielding of waxy crude oils. Ind. Eng. Chem. Res., 37:1551-1559, 1998. 2.1, 2.4.3, 3.2, 3.4.1, 4.3.1, 4.3.4, 4.3.4, 4.4, 5

[53] CHANG, C.; BOGER, D. V. ; NGUYEN, Q. D.. Influence of thermal history on the waxy structure of statically cooled waxy crude oil. SPE Journal, 5(2):148-157, 2000. 2.1, 2.4.2, 2.4.3, 3.2

[54] KANÉ, M.; DJABOUROV, M. ; VOLLE, J.-L.. Rheology and structure of waxy crude oils in quiescent and under shearing conditions. Fuel, 83:1591-1605, 2004. 2.1, 2.4.3

[55] DIMITRIOU, C. J.; MCKINLEY, G. H. ; VENKATESAN, R.. Rheo-piv analysis of the yielding and flow of model waxy crude oils. Energy \& Fuels, 2011. 2.1, 2.4.3, 2.5.4, 3.1

[56] VENKATESAN, R.; ÖSTLUND, J.-A.; CHAWLA, H.; WATTANA, P.; NYDÉN, M. ; FOGLER, H. S.. The effect of asphaltenes on the gelation of waxy oils. Energy \& Fuels, 17:1630-1640, 2003. 2.3.1

[57] RØNNINGSEN, H. P.; BJØRNDAL, B.; HANSEN, A. B. ; PEDERSEN, W. B.. Wax precipitation from north sea crude oils. 1. crystallization and dissolution temperatures, and newtonian and non-newtonian flow properties. Energy \& Fuels, 5:895-908, 1991. $2.4 .2,2.4 .3,2.4 .5$

[58] WEBBER, R. M.. Yield properties of wax crystal structures formed in lubricant mineral oils. Ind. Eng. Chem. Res., 40:195-203, 2001. 2.4.2, 3.2, 4.3.1, 4.3.1, 4.3.2

[59] BARNES, H. A.. Measuring the viscosity of large-particle (and flocculated) suspensions - a note on the necessary gap size of rotational viscometers. J. Non-Newtonian Fluid Mech., 94:213-217, 2000. 2.4 .4

[60] FERRIS, S. W.; COWLES, H. C.. Crystal behavior of paraffin wax. Ind. Eng. Chem., 37(11):1054-1062, 1945. 2.4.4

[61] BARNES, H. A.. A review of the slip (wall depletion) of polymer solutions, emulsions and particle suspensions in viscometers: its cause, character, and cure. J. Non-Newtonian Fluid Mech., 56:221251, 1995. 2.5.4, 2.5.4 
[62] YOSHIMURA, A.; PRUD'HOMME, R. K.. Wall slip corrections for couette and parallel disk viscometers. J. Rheol., 32(1):53-67, 1988. 2.5 .4

[63] NGUYEN, Q. D.; BOGER, D. V.. Yield stress measurement for concentrated suspensions. J. Rheol., 27(4):321-349, 1983. 2.5 .4

[64] NGUYEN, Q. D.; BOGER, D. V.. Direct yield stress measurement with the vane method. J. Rheol., 29(3):335-347, 1985. 2.5 .4

[65] NGUYEN, Q. D.; BOGER, D. V.. Measuring the flow properties of yield stress fluids. Annu. Rev. Fluid Mech., 24:47-88, 1992. 2.5 .4

[66] LEE, H. S.; SINGH, P.; THOMASON, W. H. ; FOGLER, H. S.. Waxy oil gel breaking mechanisms: Adhesive versus cohesive failure. Energy \& Fuels, 22:480-487, 2008. 2.5.4

[67] BRITTON, M. M.; CALLAGHAN, P. T.. Shear banding instability in wormlike micellar solutions. Eur. Phys. J., B 7:237-249, 1999. 2.5 .5

[68] LU, C. Y. D.; OLMSTED, P. D. ; BALL, R. C.. Effects of nonlocal stress on the determination of shear banding flow. Phys. Rev. Lett., 84(4):642-645, 2000. 2.5.5

[69] TINSLEY, J. F.; JAHNKE, J. P.; DETTMAN, H. D. ; PRUD'HOMME, R. K.. Waxy gels with asphaltenes 1: Characterization of precipitation, gelation, yield stress, and morphology. Energy \& Fuels, 23:2056-2064, 2009. 3.1

[70] PASO, K.; KOMPALLA, T.; OSCHMANN, H. J. ; SJOBLOM, J.. Rheological degradation of model wax-oil gels. J. Disp. Sci. Tech., $30: 472-480,2009.3 .1$

[71] $\mathrm{OH}, \mathrm{K}$; DEO, M.. Characteristics of wax gel formation in the presence of asphaltenes. Energy \& Fuels, 23:1289-1293, 2009. 3.1

[72] MAGDA, J. J.; EL-GENDY, H.; OH, K.; DEO, M. D.; MONTESI, A. ; VENKATESAN, R.. Time-dependent rheology of a model waxy crude oil with relevance to gelled pipeline restart. Energy \& Fuels, 23:1311-1315, 2009. 3.1

[73] LI, C.; YANG, Q. ; LIN, M.. Effects of stress and oscillatory frequency on the structural properties of daqing gelled crude oil at different temperatures. J. Petrol. Sci. Eng., 65:167-170, 2009. 
[74] ABIVIN, P.; HENAUT, I.; ARGILLIER, J.-F. ; MOAN, M.. Rheological behavior of foamy oils. Energy \& Fuels, 23:1316-1322, 2009. 3.1

[75] PASO, K.; SILSET, A.; GONÇALVES, M. A. L. ; SJOBLOM, J.. Characterization of the formation, flowability, and resolution of brazilian crude oil emulsions. Energy \& Fuels, 23:471-480, 2009. 3.1

[76] OH, K.; GANDHI, K.; MAGDA, J. ; DEO, M. D.. Yield stress of wax gel using vane method. Pet. Sci. Tech., 27:2063-2073, 2009. 3.1

[77] OH, K.; JEMMETT, M. ; DEO, M.. Yield behavior of gelled waxy oil: Effect of stress application in creep ranges. Ind. Eng. Chem. Res., 48:8950-8953, 2009. 3.1

[78] TAIWOL, E. A.; FASESAN, S. O. ; AKINYEMI, O. P.. Rheology of doped nigerian niger-delta waxy crude oil. Pet. Sci. Tech., 27:1381-1393, 2009. 3.1

[79] HOU, L.; ZHANG, J. ; SUN, L.. Change of yield stress of daqing crude oil with thermal and shear history. Pet. Sci. Tech., 27:21682176, 2009. 3.1

[80] HOU, L.; ZHANG, J.. A study on creep behavior of gelled daqing crude oil. Pet. Sci. Tech., 28:690-699, 2010. 3.1

[81] ELSAYED, A. Z. A.; EL-SHIEKH, T. M.. Effect of cooling rate on the flow behavior of waxy crude oils. Energy Sources, Part A, 32:197-207, 2010. 3.1

[82] DE Oliveira, M. C. K.; DE CARVAlHO, R. M.; DE CARVAlho, A. B. M.; DO COUTO, B. C.; FARIA, F. R. D. ; CARDOSO, R. L. P.. Waxy crude oil emulsion gel: Impact on flow assurance. Energy \& Fuels, 24(4):2287-2293, 2010. 3.1

[83] YI, S.; ZHANG, J.. Relationship between waxy crude oil composition and change in the morphology and structure of wax crystals induced by pour-point-depressant beneficiation. Energy \& Fuels, 25:1686-1696, 2011. 3.1

[84] YI, S.; ZHANG, J.. Shear-induced change in morphology of wax crystals and flow properties of waxy crudes modified with the pour-point depressant. Energy \& Fuels, 25, 2011. 3.1 
[85] LIN, M.; LI, C.; YANG, F. ; MA, Y.. Isothermal structure development of qinghai waxy crude oil after static and dynamic cooling. J. Pet. Sci. Eng., 77:351-358, 2011. 3.1

[86] OH, K.; DEO, M. D.. Yield behavior of gelled waxy oil in water-inoil emulsion at temperatures below ice formation. Fuel, 90:21132117, 2011. 3.1

[87] HOU, L.. Experimental study on yield behavior of daqing crude oil. Rheol. Acta, 2012. 3.1

[88] ALCAZAR-VARA, L. A.; GARCIA-MARTINEZ, J. A. ; BUENROSTROGONZALEZ, E.. Effect of asphaltenes on equilibrium and rheological properties of waxy model systems. Fuel, 93:200-212, 2012. 3.1

[89] DE OLIVEIRA, M. C. K.; TEIXEIRA, A.; VIEIRA, L. C.; DE CARVALHO, R. M.; DE CARVALHO, A. B. M. ; DO COUTO, B. C.. Flow assurance study for waxy crude oils. Energy \& Fuels, 26, 2012. 3.1

[90] ZHAO, Y.; KUMAR, L.; PASO, K.; ALI, H.; SAFIEVA, J. ; SJOBLOM, J.. Gelation and breakage behavior of model wax-oil systems: Rheological properties and model development. Ind. Eng. Chem. Res., 51(23):8123-8133, 2012. 3.1

[91] RAMIREZ-GONZALEZ, P. V.; AGUAYO, J. P.; NONES CISNEROS, S. E. Q. ; DEITERS, U. K.. Non-newtonian viscosity modeling of crude oils - comparison among models. Int. J. Thermophys., 30:1089-1105, 2009. 3.1

[92] GHANAEI, E.; MOWLA, D.. Prediction of waxy oil rheology by a new model. Energy \& Fuels, 24:1762-1770, 2010. 3.1

[93] WACHS, A.; VINAY, G. ; FRIGAARD, I.. A 1.5d numerical model for the start up of weakly compressible flow of a viscoplastic and thixotropic fluid in pipelines. J. Non-Newtonian Fluid Mech., 159:81-94, 2009. 3.1

[94] DE SOUZA MENDES, P. R.; DE ABREU SOARES, F. S.-M.; ZIGLIO, C. M. ; GONÇALVES, M. A. L.. Startup flow of gelled crudes in pipelines. J. Non-Newtonian Fluid Mech., 179-180:23-31, 2012. 3.1

[95] BARNES, H. A.. The yield stress - a review or ' $\pi \alpha \nu \tau \alpha \rho \epsilon \iota$ ' everything flows? J. Non-Newtonian Fluid Mech., 81:133-178, 1999. 3.2 
[96] GILL, F.; RUSSELL, R. J.. Pumpability of residual fuel oils. Ind. Eng. Chem., 46(6):1264-1278, 1954. 3.2

[97] BILLINGTON, E. W.. Some measurements of the time dependence of the viscosity of thixotropic fluids. Proc. Phys. Soc., 75(481):4050, 1960. 3.2

[98] BARNES, H. A.. Thixotropy - a review. J. Non-Newtonian Fluid Mech., 70:1-33, 1997. 3.2

[99] KRAYNIK, A. M.. Er fluid standards: Comments on er fluid rheology. Proc. 2nd Int. Conf. ER Fluids, 1990. 3.2

[100] HOUWINK, R.. Elasticity, Plasticity and Structure of Matter. Roelof Houwink, 1971. 3.2

[101] BONNECAZE, R. T.; BRADY, J. F.. Yield stresses in electrorheological fluids. J. Rheol., 36(1):73-115, 1992. 3.2 